\title{
24. LEG 11 MEASUREMENTS OF PHYSICAL PROPERTIES IN SEDIMENTS OF THE WESTERN NORTH ATLANTIC AND THEIR RELATIONSHIP TO SEDIMENT CONSOLIDATION
}

\author{
F. J. Paulus, The Superior Oil Company, Houston, Texas
}

\section{INTRODUCTION}

Increasing emphasis on the utilization of the sea floor for installations of submarine oil field production equipment and for mining operations has created a strong demand for information on the physical properties of submarine sediments. Investigation of these physical properties has been accelerating in response to this demand. Good summaries of the state of the art are found in recent papers by Hamilton (1970), and Keller and Bennett (1970). Both papers are good source references to other major works on the subject of physical properties of marine sediments.

The physical property measurements for the sediments recovered at Leg 11 drill sites and the relationship of these measurements to sediment consolidation are discussed in this chapter. The measurements are listed below in the numerical order in which they appear from left to right in the six columns of the physical property pages in the Site Reports of this volume.

1. Natural gamma radiation (counts $/ 7.6 \mathrm{~cm} /$ $1.25 \mathrm{~min}$.).

2. Consolidation (penetrometer; centimeters of penetration).

3. Grain size (wt\%; cumulative plot of clay, silt, sand).

4a. Water content (wt\%).

4b. Porosity (vol. \%).

5. Wet-bulk density (g/cc).

6. Sonic velocity $(\mathrm{km} / \mathrm{sec})$.

Measurements were made on board ship for all of the above except grain size. Porosity and wet-bulk density values derived from shore-based computer processing of shipboard GRAPE (Gamma-Ray Attenuation Porosity Evaluator) data, as well as grain densities from the grain-density computer program, are also included in this chapter. Continuous plots of porosity and wetbulk density, derived from the computer processing of the digitized shipboard GRAPE record, are shown in columns 4 and 5 of the physical property pages in the
Site Reports of this volume. Shipboard measurements of the salinity, $\mathrm{pH}$, and Eh of interstitial water samples, as well as, gas sample analyses derived from shipboard gas chromatograms are also tabulated and discussed in this chapter.

The physical property measurements, sediment consolidation, interstitial water analyses and gas chromatograms, and the geologist's grain density computer program are discussed in individual sections of this chapter according to the following outline:

II. Natural Gamma Radiation

III. Grain Size

IV. Porosity and Water Content

V. Grain Density Computer Program for Leg 11 Sediments

VI. Wet-Bulk Density

VII. Velocity

VIII. Sediment Consolidation

IX. Interstitial Water

X. Gas Chromatography

A bibliography containing not only cited references but additional works as well, is also included.

\section{NATURAL GAMMA RADIATION}

\section{Introduction}

Natural gamma-ray measurements were made on 1.5 -meter core sections by using a natural gamma-ray scan system based on a design provided by the Marathon Oil Company (Denver Research Center). This core gamma system is described and figured in DSDP Volume 2 (Peterson et. al., 1970).

The gamma-rays measured come from two main sources, the atmosphere, and the sediments. The core gamma system recorded the combined count from 
both sources on a strip chart, an example of which is shown in Figure 1. The strip chart is a series of records which were continuous for each 1.5-meter section of core. Gamma-ray response for test standards consisting of core liners filled with air and with water was recorded on the charts for the purpose of determining the level of the atmospheric (background) count. The level of the background, or atmospheric count, remained fairly constant (about 100 counts) throughout the analysis and is shown by a line designated " $\mathrm{BC}$ " in Figure 1.

\section{Core Gamma Records for Leg 11 Cores}

On Leg 11 a 7.5-centimeter increment of core was scanned for a count time of 1.25 minutes. Many times, the scanned interval extended beyond the end of the core material and in these cases the total count would be slightly lower. This minor reduction in count is apparent on most of the plots in column 1 of the physical property pages. A lower count would also be anticipated, for similar reasons, in the vicinity of the empty spaces which were caused by segmentation of the core during recovery. These empty spaces, or voids, are particularly abundant in the cores from Sites 102 , 103 , and 104 where they were caused by the expansion of methane gas from within the sediment. For the voids, especially the larger ones, the gamma-ray counter registered a value approximating the atmospheric count which was generally about 1600 counts less than the average values for the clay sediments. Examples of where the gamma-ray plot shows only the background count can be seen on the physical property pages for Site 103: in Core 4, where the low count in the upper 50 centimeters of Section 1 correlates with a large void in the core liner; and, in Core 5, where the low count in the upper 100 centimeters of Section 1 correlates with another large void. Figure 1 shows the effects of these voids on the strip chart record for Site 103. These examples of gamma-ray count being reduced by the presence of voids are only two of the scores of similar instances which can be identified on the gamma-ray plot in column 1 of the physical property pages by simply correlating the details of core recovery with the gamma-ray plot. Occasional discrepancies occur in the correlations between the gamma-ray plot and the core recovery shown in the lithology column of the corresponding core page. This was caused by the rearrangement of core segments during the opening of the core liner subsequent to the gamma-ray logging.

\section{Gamma-Ray Response}

About half of all gamma radiation emitted from sediments comes from the radioactive isotope of potassium $\left(\mathrm{K}^{40}\right)$. The remainder comes principally from the radioactive isotopes of uranium and thorium. In general, clays adsorb large amounts of cations and among them potassium is one of the more abundant

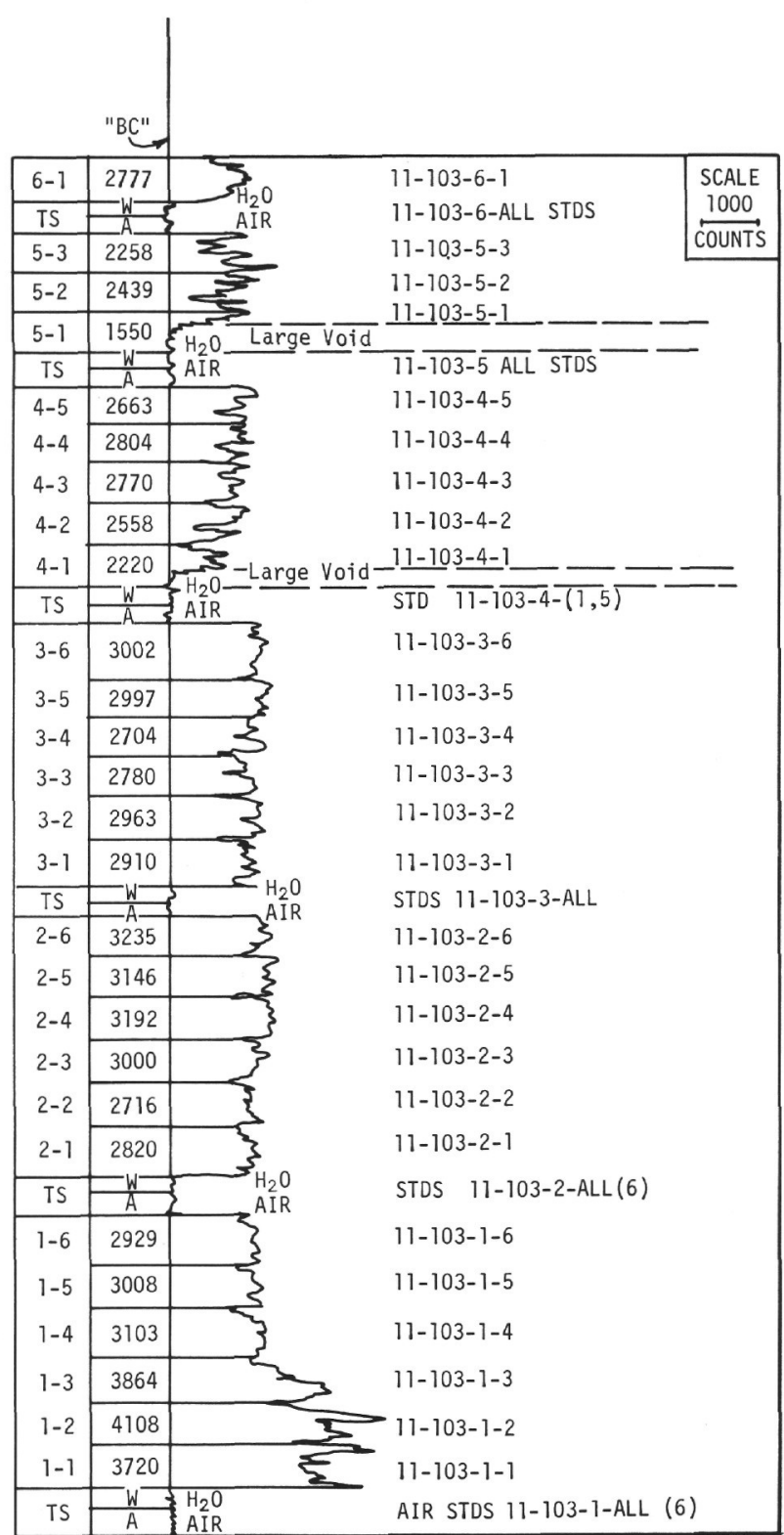

Figure 1. Gamma-ray record for Site 103. Intervals showing measurements of background count based on test standards are labeled TS and include an air standard $(A)$ and a water standard $(W)$. The level of the background count is shown by the line designated BC. Core and section numbers are plotted on left margin. The 4-digit numbers plotted near the left margin represent the average total gamma-ray count per section of core. These average values form the basis for the plot shown in column 1 of the physical property pages in the Site Reports (this volume). The numerous larger excursions of the curve for cores 4 and 5 are caused by the presence of numerous voids due principally to methane gas expansion. Smaller and fewer gas voids occur in cores 3 and 6. There were no voids in cores 1 and 2 . 
cations available during the early diagenetic history of a clay deposit (see Engelhardt and Gaida, 1963). The adsorption of potassium and calcium ions relative to late Tertiary and Quaternary clays of the upper continental slope was recently discussed by Perry (1970). Only 0.01 per cent of all of the potassium ions are radioactive, but because of the larger number of potassium ions involved a significant amount of the radioactive isotope is accumulated in clays or shales. Basically, therefore, the gamma-ray log distinguishes them from other formations.

\section{Examples of Gamma-Ray Response to Lithologic Change}

The influence which major lithologic changes have on gamma-ray response is illustrated on the strip chart record for Site 105 (Figures 4 and 5). Figure 4 shows the change in gamma-ray count which accompanies the change in lithology from carbonaceous zeolitic clay, where the gamma-ray count averaged 1700 (background removed). These averages are measured from the background level (line "BC") to the peak average (line "PA") in Figure 4. The remainder of the limestone section at Site 105 has a gamma-ray count (background removed) ranging from a lower average limit of about 300 to an upper average limit of about 700 .

Figure 5 shows another good example of how the core gamma can be used to identify changes in lithology. This figure shows that the change in lithology from a limestone to a calcareous claystone at Site 105 (Core 33) is accompanied by a significant increase of about 600 counts on the gamma-ray strip chart record. The count gradually increases downhole (towards top of figure) which would suggest a gradual increase in clay content in the older portion of the claystone. Although described as a clayey limestone in the Site Report (this volume), the gamma-ray record and the carbonate analyses would suggest the use of the term calcareous claystone for the interval below Section 1, Core 33 (Figure 5). Table 3 shows that Cores 35 through 39 have average gamma-ray counts similar to the average for clay sediments from Leg 11 core holes. In addition, Cores 38 and 39 also have low carbonate percentages and are therefore described simply as claystone in Tables 1 and 3 .

An example of the influence which a change in the carbonate/clay ratio can have on the gamma-ray response in an otherwise essentially homogeneous clay lithology is seen in Figure 6. This figure is a portion of the strip chart record for Hole 101A. The first two sections in Core 2 show a peak average count of 1600 for clay containing 30 per cent carbonate, whereas, the lower four sections average 9 per cent carbonate and have a peak average of 2300 counts. The carbonate values were taken from shore based laboratory analyses.

\section{Lithologies and Average Gamma-Ray Count}

Table 1 lists the average gamma-ray count (background removed) for the principal lithologies encountered at six of the eleven drill sites. Clays had a core average range of 800 to 2300 for an average count of about 1600 to 1700 . Claystone averaged 1600 counts, while calcareous claystone had a core average range of 1000 to 1700 which averaged 1402 . Limestone had a core average range of 300 to 800 which averaged 545 . The core averages were based on the peak average line drawn on the strip chart records (see Figures 4, 5, and 6 for illustration of peak average line).

\section{Gamma-Ray Plots Compared with Grain-Size Plots}

Since natural gamma-ray measurements relate primarily to clay content, the plot in column 1 of the physical property pages should show a relationship with the grain size plot in column 3. A significant reduction in the clay-sized fraction should be accompanied by a corresponding reduction in the gamma-ray values because of the arrangement of scales in columns 1 and 3 on the physical property pages. The clay fraction curve should, in general, migrate in unison with the gamma-ray curve; however, due to the homogeneity of most cores and the sampling frequency for grain-size analysis there are no such examples of this relationship. The gamma-ray and grain-size plots for the near sea-floor anomalies at Sites 103 and 104 show little or no correlation.

\section{Gamma-Ray Anomalies in Leg 11 Cores}

The relatively strong radioactivity anomalies at Site 105 (Core 9) and Site 104 (Core 1), and the lesser anomaly at Site 103 (Core 1) could be useful markers for correlation purposes in future core hole programs in the vicinity of these sites. The anomalies are summarized in Table 2 and are discussed in the following paragraphs.

The anomaly at Site 105 has a peak value of 7500 counts (background removed) at a sub-seafloor depth of 290.7 meters which is nearly coincident with the top of the black carbonaceous zeolitic clay which extends from 290 meters (105-9-3, 100 centimeters) to 403 meters (105-17-1,20 centimeters). The anomaly is probably related to a local concentration of organic carbon in the black carbonaceous clay. The organic carbon analysis at 290.65 meters $(105-9-4,15$ centimeters) produced the highest value ( 14.8 per cent) for Leg 11 samples. The remainder of the black clay from the base of the gamma-ray anomaly in Core 9 to the top of Core 17 had an organic carbon content range of 0.3 to 4.8 with an average of 1.9 per cent. The multicolored clay overlying the black clay in Core 9 had an average organic carbon content of 0.1 per cent. For all other Leg 11 analyses only two values, 3.3 


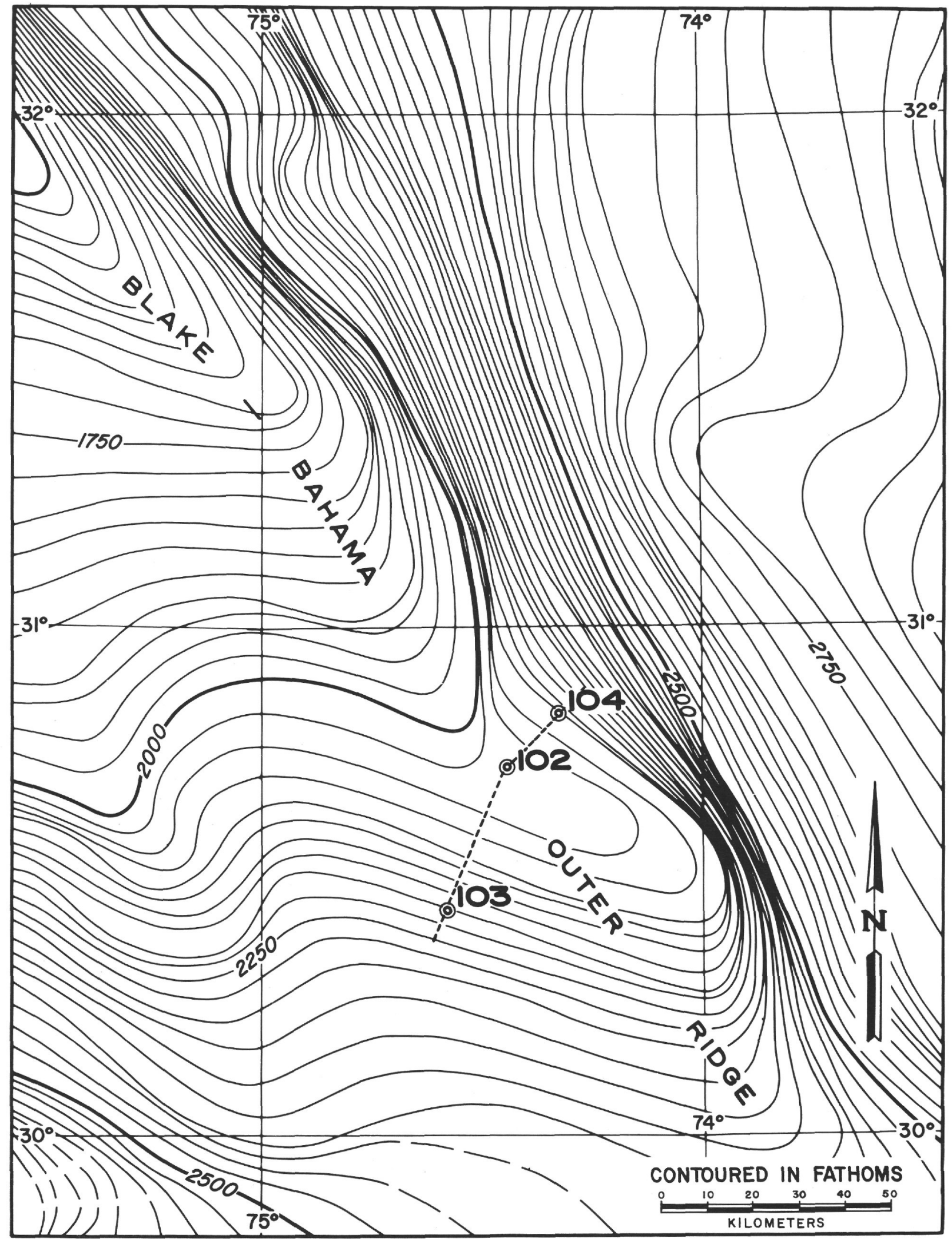

Figure 2. Index map showing location of cross section through D.S.D.P. Sites 102, 103, 104. The section appears in Figure 3. Contours taken from bathymetric map compiled by Belding and Holland (1970). 


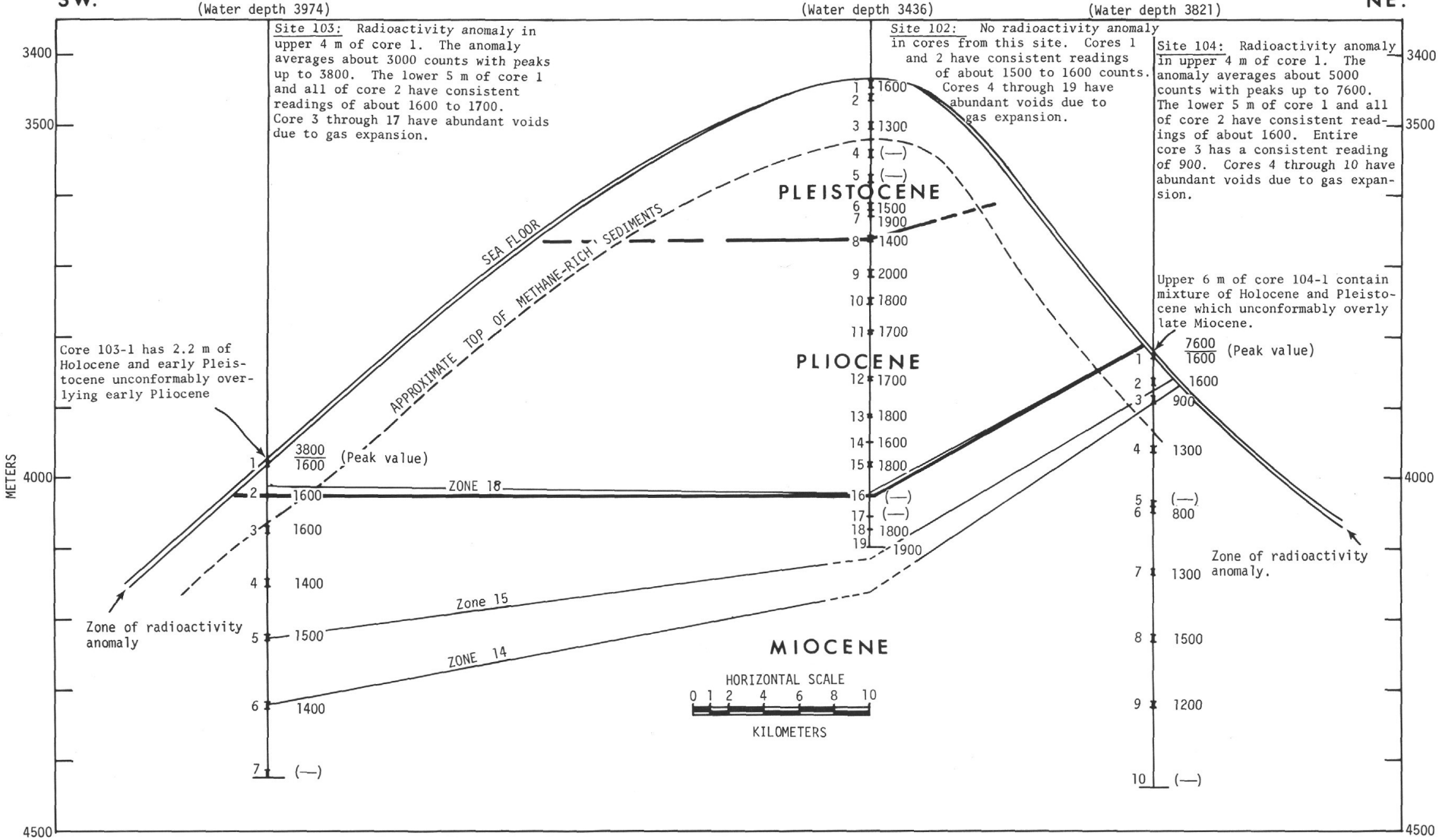

Figure 3. Cross section through Blake-Bahama Outer Ridge section showing relationships of gamma-ray count with physiography of sea floor, occurrence of methane-rich sediments, and biostratigraphic correlations based on foraminifers. Note that the near-sea floor radioactivity anomaly is associated with a major erosional surface of early Pleistocene/early Pliocene age at Site 103 and of Pleistocene/Miocene age at Site 104. There is no radioactivity anomaly at Site 102 where the depositional record is complete. Core numbers appear on the left side of each core hole. The approximate average gamma-ray count per core is shown on the right side of each core hole. The peak values of the radioactivity anomalies in core 1 at Sites 103 and 104 are also shown. The second value posted for core 1 is the average of the core below the anomaly. The background count has been removed. The average values were obtained from a smoothing curve drawn through the peak readings on the strip chart record thus eliminating most of the effect of low values caused by voids and overruns at section ends. All depths are drill pipe measurements. Depth datum is derrick floor (10 m above sea level). Vertical exaggeration - 40X. See Figure 2 for location of section. 


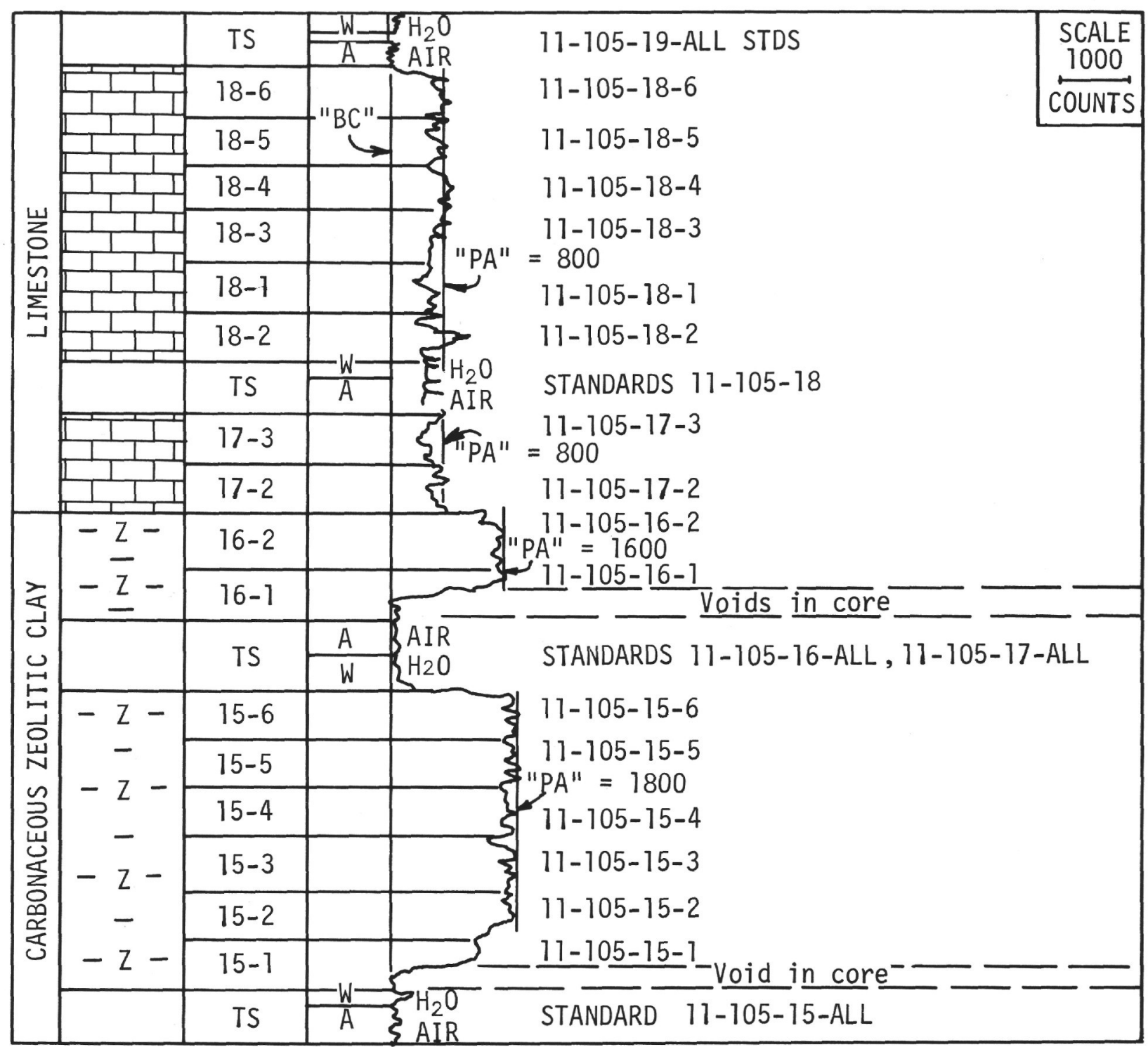

Figure 4. Gamma-ray strip chart record for a portion of Site 105. Lithology columns are on far left. Core and section numbers are in column adjacent to strip chart. Intervals showing measurements of background, or atmospheric, count based on test standards $(A=$ air; $W=$ water) are labeled $T S$. " $B C$ " designates the line representing the level of the background count. "PA" designates the peak average line. Note that the major lithologic change between cores 16 and 17 is accompanied by a corresponding marked change in the gamma-ray count.

(Core 105-17) and 2.7 (Core 105-18) exceeded an otherwise upper limit of 1.8 per cent for the range of organic carbon values. Since carbon generally attracts cations, a local concentration of organic carbon such as occurs at the top of the black clay in Core 9 could be the principal factor in bringing about the concentration of significant amounts of the radioactive isotopes of potassium, uranium, and thorium. The localized concentration of these isotopes would result in the exceptionally high gamma-ray count at the top of the black clay in Core 9 at Site 105.
The near-sea floor anomalies at Site 103 and 104 are confined to sediments containing Pleistocene fauna. The peaks of the anomalies are in close proximity to major erosional surfaces. The peak of the Site 103 anomaly registered 3800 counts at 2.3 meters beneath the sea floor. The peak occurs immediately above the base of the anomaly. The base is coincident with an early Pleistocene/early Pliocene unconformity. The anomaly begins about at the sea floor and is confined entirely to Pleistocene sediments. The holocene at Site 103 is present only as a thin veneer about 2 centimeters thick. 


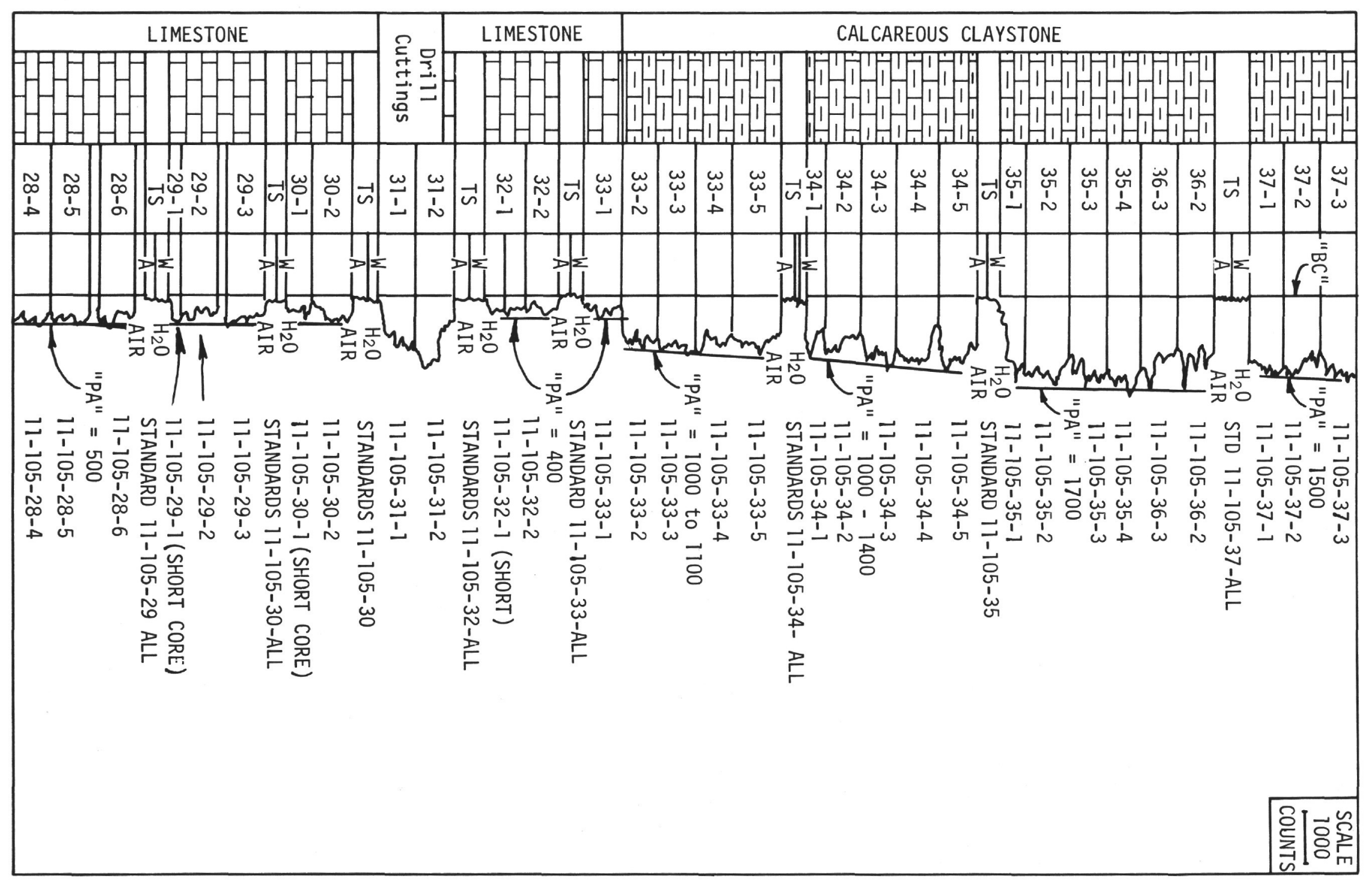

Figure 5. Gamma-ray strip chart record for a portion of Site 105 showing the gamma-ray count in limestone changing to a higher count in calcareous claystone (Core 33). Core and section numbers are in the column adjacent to the strip chart. Intervals showing measurement of background, or atmospheric count based on test standards ( $A=$ air; $W=$ water) are labeled TS. "BC" designates the line representing the level of background count. "PA" designates the peak average line. The count increases down hole (toward top of Figure) below core 33-2 due to a gradual increase in clay content in the older beds. Cores 33-2 through 37 have been described elsewhere as clayey limestone (Site Report, this volume). See text (this chapter) for lithology discussion. 


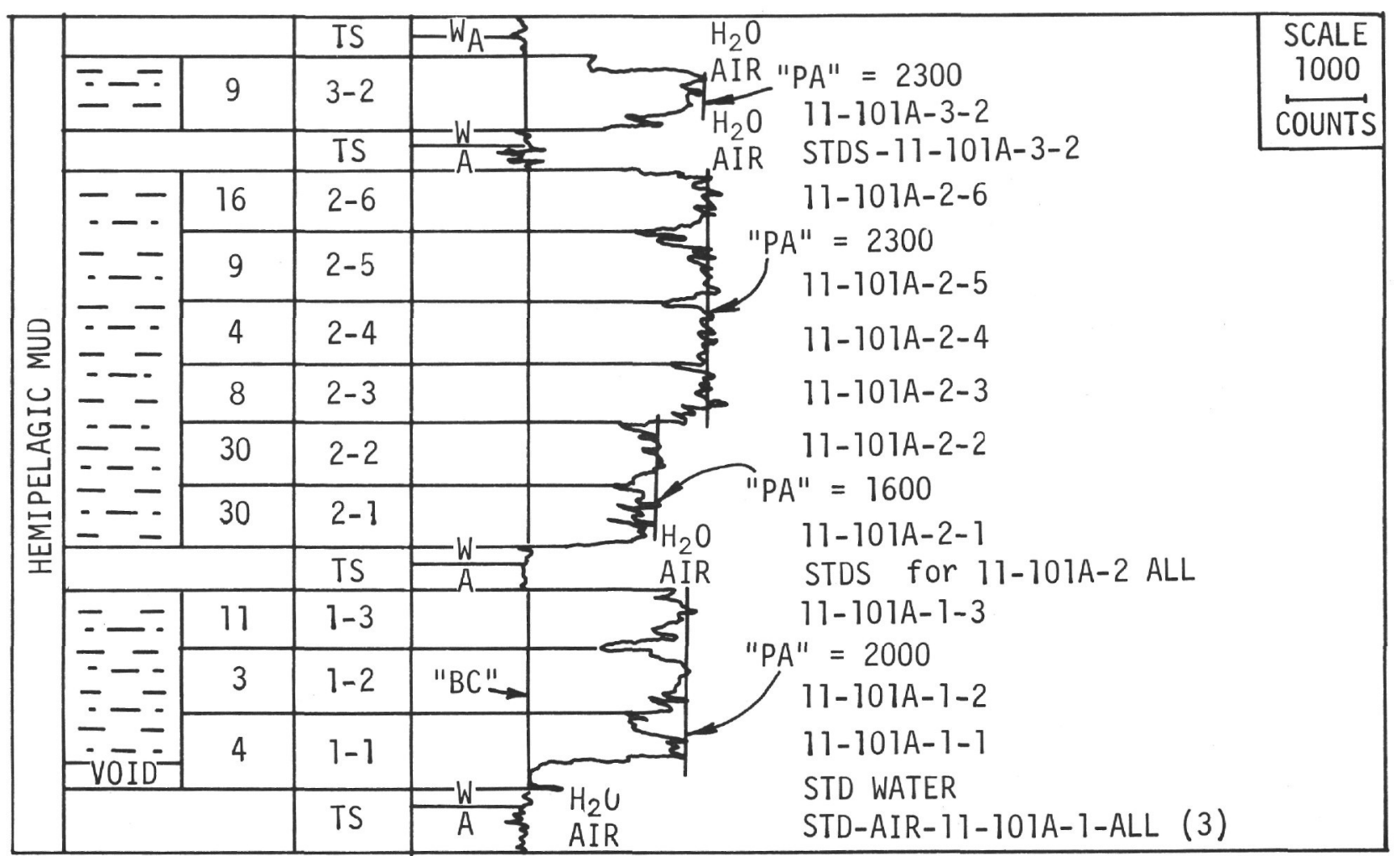

Figure 6. Gamma-ray strip chart record for a portion of Site $101 \mathrm{~A}$ illustrating the influence which a change in the carbonate/clay ratio can have on the gamma-ray response in an otherwise essentially homogeneous clay lithology (Core 2). Percentage of carbonate in the sediment is shown to the left of the core and section column adjacent to the left margin of the strip chart. The carbonate values are from the shore based carbon/carbonate analyses. Lithology columns are on far left. Intervals showing measurements of background, or atmospheric, count based on test standards ( $A=$ air; $W=$ water) are labeled $T S$. "BC" designates the line representing the level of background count. "PA" designates the peak average line which is used in conjunction with the "BC" line to determine the average gamma-ray count per interval of sediment.

The anomaly at Site 104 can be divided into two parts with one peak of about 6000 counts at 40 centimeters below the sea floor and another peak of 7600 counts occurring just above the base of the anomaly at a depth of about 3 meters. Both of these peaks occur within a zone which appears to have been disturbed by drilling with the result that Pleistocene sediments have been intruded with Holocene material. The first 7 centimeters below the sea floor is definite Holocene. The base of the mixed Holocene-Pleistocene zone is at 5.35 meters and is in contact with sediments of definite late Miocene age.

Although the gamma-ray anomalies at Sites 103 and 104 are confined to Pleistocene sediments, they are most probably related to phemonena associated with the major erosional unconformities encountered at these sites. This postulation is supported by the fact that radioactivity anomalies were absent in cores from Site 102 in spite of the fact that a complete Pleistocene sequence was encountered at the site. Coring at Site 102 encountered a normal transitional HolocenePleistocene boundary beginning at one meter below the sea floor. Normal Pleistocene-Pliocene and PlioceneMiocene boundaries were encountered at 224 meters and 585 meters, respectively (Figure 3). The absence of an anomaly at Site 102 appears to be related to the absence of major erosional surfaces at Site 102.

Gamma-ray anomalies have been used to indicate the presence of certain types of economic ore deposits. Radioactivity levels indicative of economic mineral deposits would probably be tens of times larger than the gamma-ray counts for the anomalies at Sites 103, 104 and 105. No economic ore deposits are indicated by gamma-ray measurements made on Leg 11 cores.

\section{GRAIN SIZE}

\section{Introduction}

Grain size determinations were made on samples from Leg 11 cores at the shore based laboratory at Scripps Institution of Oceanography. Three grain-size fractions (sand, silt and clay) were determined as weight percentages of a unit volume. These percentages are 
TABLE 1

Average Gamma-Ray Count for Principal Lithologies in Leg 11 Cores.

Based on Strip Chart Records for Sites 99 (Hole A), 101, 102, 103, 104, 105

\begin{tabular}{lcccc}
\hline \multicolumn{1}{c}{ Lithology } & $\begin{array}{c}\text { Carbonate Rangea } \\
(\%)\end{array}$ & $\begin{array}{c}\text { Average Gamma-Ray } \\
\text { Count } b\end{array}$ & $\begin{array}{c}\text { Range of } \\
\text { Core Averages }\end{array}$ & $\begin{array}{c}\text { Number of } \\
\text { Core Records }^{\mathrm{d}}\end{array}$ \\
\hline Clay & $0-14$ & 1600 & $800-2200$ & 7 \\
Silty clay & $0-23$ & 1702 & $1200-2300$ & 41 \\
Calcareous silty clay & $25-57$ & 1600 & $1400-2000$ & 7 \\
Claystone & $18-19$ & 1600 & 1600 & 2 \\
Calcareous claystone $^{\mathrm{f}}$ & $26-49$ & 1420 & $1000-1700$ & 5 \\
Limestoneg & $59-93$ & 545 & $300-800$ & 17 \\
\hline
\end{tabular}

Note: This table is a summary of data listed in Table 3. See text (this chapter) for lithology discussion of clayey limestone.

\footnotetext{
${ }^{a}$ Range of core averages. Derived from shore based laboratory carbonate analyses. The carbonate percentage is based on the results of the carbon analysis. Total carbon and organic carbon (carbon remaining after treatment with hydrochloric acid) are determined in terms of per cent by weight. The theoretical per cent calcium carbonate in the sediment sample is calculated as follows: (\% total $\mathrm{C}-\% \mathrm{C}$ after acidification) $\times 8.33=$ per cent calcium carbonate $\left(\mathrm{CaCO}_{3}\right)$. In reality, the carbonate value may not be entirely $\mathrm{CaCO}_{3}$ but may include contributions from other carbonates, such as, $\mathrm{MgCO}_{3}$ (dolomite) or $\mathrm{FeCO}_{3}$ (siderite). Precision of these data is as follows:

$$
\begin{aligned}
\text { Total carbon (within } 1.2 \text { to } 12 \%) & = \pm 0.3 \% \text { absolute } \\
\text { (within } 0 \text { to } 1.2 \%) & = \pm 0.06 \% \text { absolute } \\
\text { Organic carbon } & = \pm 0.06 \% \text { absolute } \\
\text { Calcium carbonate (within } 10-100 \%) & = \pm 2.5 \% \text { absolute } \\
\text { (within } 0-10 \%) & = \pm 0.5 \% \text { absolute }
\end{aligned}
$$

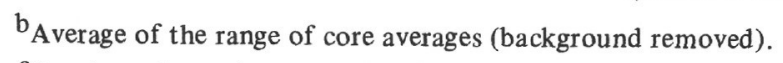

${ }^{{ }}$Based on the peak average line drawn on the strip chart record. See Figures 4, 5, and 6 for illustration of peak average line.

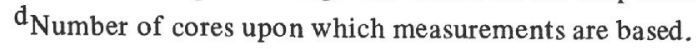

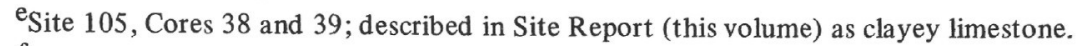

$\mathrm{f}_{\text {Site }}$ 105, Cores 33-2 through 37; described in Site Report (this volume) as clayey limestone.

\begin{tabular}{|c|c|c|c|c|c|}
\hline \multicolumn{2}{|c|}{ Site-Core (Section) } & \multirow{2}{*}{$\frac{\text { Lithology }}{\text { Hemipelagic mud }}$} & \multirow{2}{*}{$\frac{\begin{array}{c}\text { Carbonate } \\
\text { Range }^{\mathrm{a}}\end{array}}{18-35}$} & \multirow{2}{*}{$\frac{\begin{array}{c}\text { Organic Carbon } \\
\text { Range }^{\mathrm{a}}\end{array}}{0.1-0.4}$} & \multirow{2}{*}{$\begin{array}{c}\begin{array}{c}\text { Range of Gamma-Ray } \\
\text { Count } b\end{array} \\
2300-3800\end{array}$} \\
\hline $103-1$ & $(1-3)$ & & & & \\
\hline $104-1$ & $(1-3)$ & Hemipelagic mud & $2-59$ & $0.1-0.5$ & $4000-7600$ \\
\hline $105-9$ & (4) & Black clay & 4 & 14.8 & $2000-7500$ \\
\hline
\end{tabular}

$\mathrm{g}_{\text {Site } 105}$, Cores 17,18 , and 20 through 33-1.
}

TABLE 2

Gamma-Ray Anomalies Encountered at Leg 11 Sites

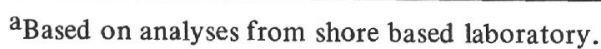

${ }^{b}$ Taken from strip chart record. Background count removed. See Figure 4 for strip chart record of Site 103. 
TABLE 3

Average Gamma-Ray Count Per Core Based on Sites 99 (Hole A), 101, 102, 103, 104, 105

\begin{tabular}{|c|c|c|c|c|c|c|c|c|}
\hline \multicolumn{9}{|c|}{ Lithologya } \\
\hline \multicolumn{3}{|c|}{ Hole-Core (Section) } & \multirow{2}{*}{$\frac{\mathrm{G} \mathrm{Z}}{\text { Silty clay }}$} & CD & $\mathrm{CO}_{3}$ & $\begin{array}{c}\text { Gamma-Ray } \\
\text { Count } b\end{array}$ & Age & \multirow[t]{2}{*}{ Remarks } \\
\hline $99 \mathrm{~A}$ & & $(1-5)$ & & $\begin{array}{l}\text { Foraminiferal- } \\
\text { nannofossil }\end{array}$ & 31.0 & 1400 & Pleistocene & \\
\hline $99 \mathrm{~A}$ & & $(1-6)$ & Silty clay & $\begin{array}{l}\text { Foraminiferal- } \\
\text { nannofossil } \\
\text { ooze }\end{array}$ & 22.0 & 1200 & Pliocene & \\
\hline 101 & & $(1-6)$ & Silty clay & Hemipelagic mud & 12.0 & 1500 & Pliocene & \\
\hline 101 & & $(1-5)$ & Silty clay & Hemipelagic mud & 10.0 & 1600 to 2100 & Miocene & $\begin{array}{l}\text { Count gradually increases } \\
\text { with depth }\end{array}$ \\
\hline $101 \mathrm{~A}$ & & $(1-3)$ & Silty clay & Hemipelagic mud & 6.0 & 2000 & Miocene & \\
\hline $101 \mathrm{~A}$ & & $(1,2)$ & Silty clay & Hemipelagic mud & 30.0 & 1600 & Miocene & $\begin{array}{l}\text { Dolomite rhombs } \\
\text { common }\end{array}$ \\
\hline $101 \mathrm{~A}$ & & $(3-6)$ & Silty clay & Hemipelagic mud & 9.0 & 2300 & Miocene & \\
\hline $101 \mathrm{~A}$ & & (2) & Silty clay & Hemipelagic mud & 10.0 & 2300 & Miocene & \\
\hline $101 \mathrm{~A}$ & & $(1,2)$ & Silty clay & Black clay & 3.0 & 1600 & Aptian & $0.9 \%$ organic carbon. \\
\hline $101 \mathrm{~A}$ & & $(1,2)$ & Clay & Black clay & 4.0 & 2000 & Neocomian & $1.0 \%$ organic carbon. \\
\hline 102 & -1 & $(1-6)$ & Silty clay & $\begin{array}{l}\text { Foraminiferal- } \\
\text { nannofossil } \\
\text { ooze }\end{array}$ & 15.0 & 1600 & Pleistocene & \\
\hline $102-$ & & $(1-6)$ & Silty clay & Hemipelagic mud & 10.0 & 1500 & Pleistocene & \\
\hline 102 & & (2) & (NS) & Hemipelagic mud & 15.0 & 1300 & Pleistocene & \\
\hline 102 & & $(1-6)$ & Silty clay & Hemipelagic mud & 28.0 & 1500 & Pleistocene & \\
\hline 102 & & $(2-4)$ & Silty clay & Hemipelagic mud & 23.0 & 1500 & Pleistocene & \\
\hline 102 & & $(5,6)$ & Silty clay & Hemipelagic mud & 15.0 & 1900 & Pleistocene & \\
\hline 102 & -8 & $(1-5)$ & Silty clay & Hemipelagic mud & 15.0 & 1400 & $\begin{array}{l}\text { Pleistocene/ } \\
\text { Pliocene }\end{array}$ & \\
\hline 102 & -9 & $(1-6)$ & Silty clay & Hemipelagic mud & 27.0 & 2000 & Pliocene & \\
\hline 102 & -10 & $(1-6)$ & Clay \& & & & & & \\
\hline & & & silty clay & Hemipelagic mud & 14.0 & 1800 & Pliocene & \\
\hline 102 & -11 & $(1-3)$ & Silty clay & Hemipelagic mud & 18.0 & 1700 & Pliocene & \\
\hline 102 & .12 & $(1-4)$ & Silty clay & Hemipelagic mud & 21.0 & 1700 & Pliocene & \\
\hline 102 & -13 & $(1-4)$ & Silty clay & Hemipelagic mud & 16.0 & 1800 & Pliocene & \\
\hline 102 & -14 & $(1,2)$ & (NS) & Hemipelagic mud & 19.0 & 1600 & Pliocene & \\
\hline 102 & $-15)$ & (1) & (NS) & Hemipelagic mud & 20.0 & 1800 & Pliocene & \\
\hline 102 & -18 & $(1-4)$ & Clay \& & & & & & \\
\hline 102 & -19 & ( 1 ) & $\begin{array}{l}\text { silty clay } \\
\text { (NS) }\end{array}$ & $\begin{array}{l}\text { Hemipelagic mud } \\
\text { Hemipelagic mud }\end{array}$ & $\begin{array}{l}14.0 \\
11.0\end{array}$ & $\begin{array}{l}1800 \\
1900\end{array}$ & $\begin{array}{l}\text { Miocene } \\
\text { Miocene }\end{array}$ & \\
\hline 103 & -1 & $(1-3)$ & Silty clay & Hemipelagic mud & 29.0 & $2300-3800$ & Pleistocene & Anomaly \\
\hline 103 & -1 & (4-6) & Silty clay & Hemipelagic mud & 14.0 & 1600 & Pleistocene & \\
\hline 103 & -2 & $(1-5)$ & $\begin{array}{l}\text { Clay \& } \\
\text { silty clay }\end{array}$ & Hemipelagic mud & 12.0 & 1600 & $\begin{array}{l}\text { Pliocene/ } \\
\text { Miocene }\end{array}$ & \\
\hline 103 & -2 & (6) & $\begin{array}{l}\text { Clayey } \\
\text { silt }\end{array}$ & Hemipelagic mud & 57.0 & 1700 & Miocene & $\begin{array}{l}\text { Dolomite rhombs are } \\
\text { dominant }\end{array}$ \\
\hline 103 & -3 & $(1-6)$ & Silty clay & Hemipelagic mud & 4.0 & 1600 & Miocene & \\
\hline 103 & -4 & $(1-5)$ & Silty clay & Hemipelagic mud & 8.0 & 1400 & Miocene & \\
\hline 103 & -5 & (1-3) & Silty clay & Hemipelagic mud & 25.0 & 1500 & Miocene & \\
\hline 103 & -6 & (1) & Silty clay & Hemipelagic mud & 12.0 & 1400 & Miocene & \\
\hline 104 & -1 & $(1-3)$ & $\begin{array}{l}\text { Sandy } \\
\text { silty clay }\end{array}$ & Hemipelagic mud & 23.0 & $4000-7600$ & Pleistocene & Anomaly \\
\hline
\end{tabular}


TABLE 3 - Continued

\begin{tabular}{|c|c|c|c|c|c|c|c|c|}
\hline \multicolumn{3}{|c|}{ Hole-Core (Section) } & \multicolumn{3}{|c|}{ Lithologya } & $\begin{array}{l}\text { Gamma-Ray } \\
\text { Count }^{b}\end{array}$ & Age & Remarks \\
\hline 104 & -1 & $(4-6)$ & Silty clay & Hemipelagic mud & 14.0 & 1600 & Miocene & \\
\hline 104 & -2 & $(1-6)$ & Silty clay & Hemipelagic mud & 12.0 & 1600 & Miocene & \\
\hline 104 & -3 & $(1-6)$ & Clay & Hemipelagic mud & 11.0 & 900 & Miocene & $\begin{array}{l}\text { No voids; top of siliceous } \\
\text { fauna zone. }\end{array}$ \\
\hline 104 & -4 & $(1-6)$ & Clay & Hemipelagic mud & 9.0 & 1300 & Miocene & \\
\hline 104 & -6 & $(1-6)$ & Clay & Hemipelagic mud & 14.0 & 800 & Miocene & \\
\hline 104 & -7 & $(1,2,4)$ & Silty clay & Hemipelagic mud & 20.0 & 1300 & Miocene & \\
\hline 104 & -8 & $(2-5)$ & Silty clay & Hemipelagic mud & 14.0 & 1500 & Miocene & \\
\hline 104 & -9 & $(1-3)$ & Silty clay & Hemipelagic mud & 21.0 & 1200 & Miocene & \\
\hline 105 & -2 & $(1-3)$ & $\begin{array}{l}\text { Clayey } \\
\text { silt }\end{array}$ & Hemipelagic mud & 25.0 & 1500 & Pliocene & \\
\hline 105 & -3 & $(1-4)$ & Clay & Hemipelagic mud & 9.0 & 1900 & Pliocene & \\
\hline 105 & -4 & $(1-6)$ & Silty clay & Silty clay & 1.0 & 2200 & Miocene & \\
\hline 105 & -5 & $(1-3)$ & Clay & Silty clay & 0.1 & 2100 & $\begin{array}{l}\text { Early } \\
\text { Tertiary }\end{array}$ & \\
\hline 105 & -6 & $(1,2)$ & $\begin{array}{l}\text { Clay \& } \\
\text { silty clay }\end{array}$ & Silty clay & 0.0 & 2100 & $\begin{array}{l}\text { Early } \\
\text { Tertiary }\end{array}$ & \\
\hline 105 & -7 & $(1-5)$ & Clay & --- & --- & -- & -- & Hole cavings. \\
\hline 105 & -7 & $(6)$ & Silty clay & Silty clay & 0.0 & 2200 & (Barren) & \\
\hline 105 & -8 & $(1-5)$ & Silty clay & Silty clay & 0.1 & 2200 & (Barren) & \\
\hline 105 & -8 & (6) & $\begin{array}{l}\text { Clayey } \\
\text { silt }\end{array}$ & -- & -- & -- & -- & Hole cavings. \\
\hline 105 & -9 & $(1-3 \pm)$ & Clay & Silty clay & 0.0 & 2200 & Cenomanian & \\
\hline 105 & -9 & $(4)$ & $\begin{array}{l}\text { Clayey } \\
\text { silt }\end{array}$ & Black clay & 4.0 & $2000-7500$ & Cenomanian & Anomaly \\
\hline 105 & -9 & $(5,6)$ & Silty clay & Black clay & 1.0 & $2000(2800)$ & Cenomanian & Peaks in \\
\hline 105 & -10 & $(1,2)$ & Silty clay & Silty clay & 0.3 & $1800(2600)$ & Cenomanian & Peaks in \\
\hline 105 & -11 & $(1-5)$ & Silty clay & Black clay & 19.0 & $1800(2600)$ & Cenomanian & Peaks in \\
\hline 105 & -12 & $(1-4)$ & Silty clay & Black clay & 17.0 & 1600 & Albian & \\
\hline 105 & -13 & $(1-6)$ & Silty clay & -- & -- & -- & -- & Hole cavings. \\
\hline 105 & -14 & (1) & Silty clay & Black clay & 0.0 & 1500 & Albian & \\
\hline 105 & -15 & $(1-6)$ & Silty clay & Black clay & 0.8 & 1800 & Albian & \\
\hline 105 & -16 & $(1-2)$ & Silty clay & Black clay & 0.4 & 1600 & Aptian & \\
\hline 105 & -17 & $(2,3)$ & (NS) & Limestone & 71.0 & 800 & Neocomian & \\
\hline 105 & -18 & $(1-6)$ & Silty clay & Limestone & 67.0 & 800 & Neocomian & $\mathrm{GZ}=$ size term \\
\hline 105 & -19 & $(1-4)$ & Silty clay & -- & --- & --- & -- & Hole cavings. \\
\hline 105 & -20 & (1) & Clay & Limestone & (NS) & 700 & Neocomian & $\mathrm{GZ}=$ size term \\
\hline 105 & -21 & $(1,2)$ & Clay & Limestone & 72.0 & 300 & Neocomian & $\mathrm{GZ}=$ size term \\
\hline 105 & -22 & $(1,2)$ & (NS) & Limestone & 93.0 & 400 & Neocomian & \\
\hline 105 & -23 & $(1,2)$ & Clay & Limestone & 75.0 & 500 & $\begin{array}{l}\text { Jurassic- } \\
\text { Cretaceous }\end{array}$ & $\mathrm{GZ}=$ size term \\
\hline $105-$ & -24 & $(1,2)$ & (NS) & Limestone & 84.0 & 500 & $\begin{array}{l}\text { Jurassic- } \\
\text { Cretaceous }\end{array}$ & \\
\hline 105 & -25 & $(1-3)$ & (NS) & Limestone & 86.0 & 600 & $\begin{array}{l}\text { Jurassic- } \\
\text { Cretaceous }\end{array}$ & \\
\hline 105 & -26 & $(1,2)$ & (NS) & Limestone & 62.0 & 700 & $\begin{array}{l}\text { Jurassic- } \\
\text { Cretaceous }\end{array}$ & \\
\hline 105 & -27 & $(1-3)$ & (NS) & Limestone & 62.0 & 700 & $\begin{array}{l}\text { Jurassic- } \\
\text { Cretaceous }\end{array}$ & \\
\hline 105 & -28 & ( 1 ) & (NS) & Limestone & 59.0 & 700 & $\begin{array}{l}\text { Jurassic- } \\
\text { Cretaceous }\end{array}$ & $\begin{array}{l}\text { See Figure } 5 \text { for } \\
\text { Cores } 28 \text { through } 37 .\end{array}$ \\
\hline
\end{tabular}


TABLE 3 - Continued

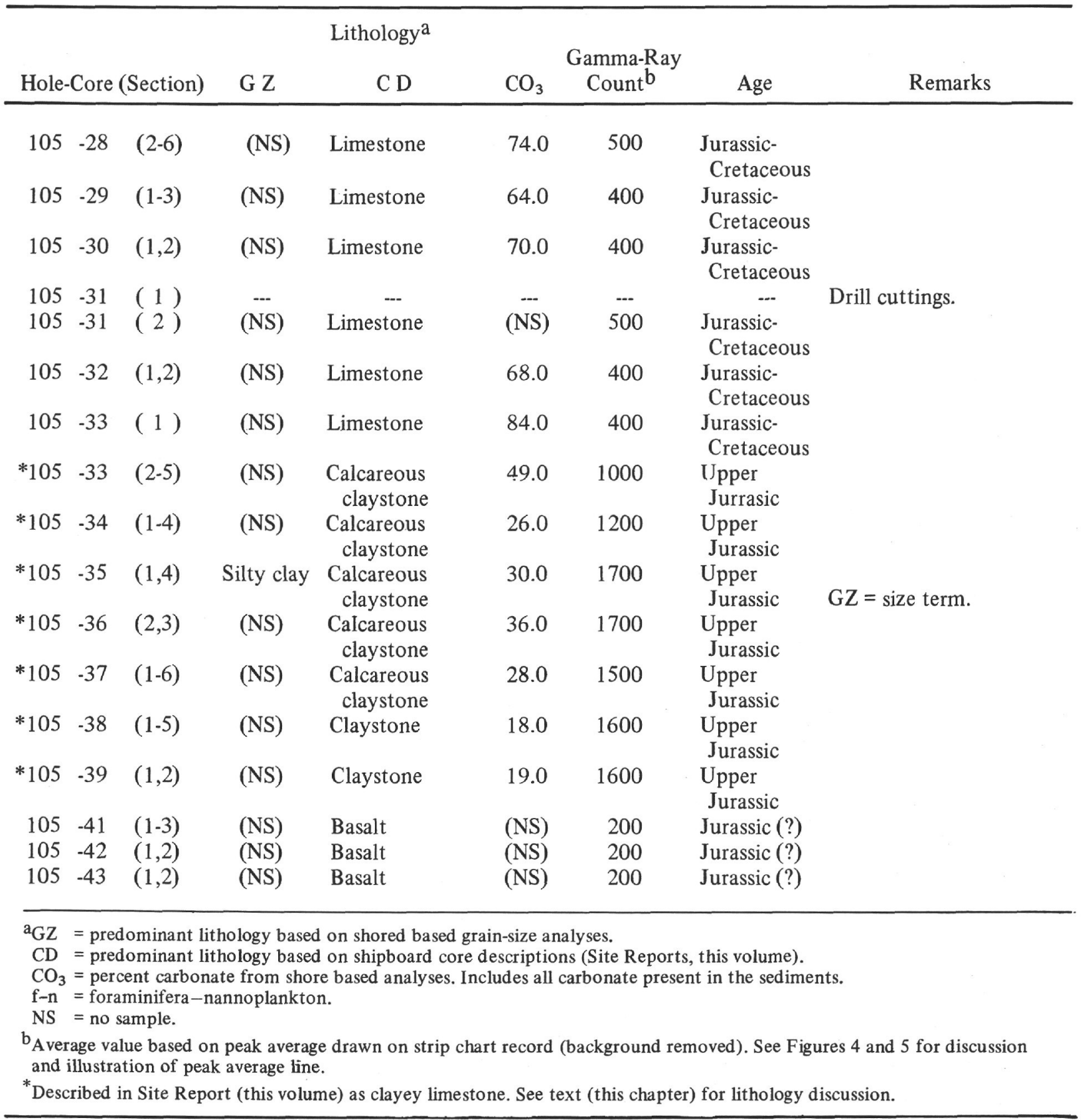

shown as a cumulative plot in column 3 of the physical property pages of the Site Reports (this volume). Details of the analytical procedures and results can be found in a separate chapter (Boyce, this volume).

\section{Grain Size-Velocity Relationship}

The relationship between grain size and sound velocity has been studied by Horn et al. (1968), Schreiber (1968), Hamilton (1970), and others. Hamilton (1970) concluded that data derived from laboratory analysis of sediment size have been found to be among the best indices for empirical derivations of sound velocity. A discussion of the grain size-velocity relationships can be found in the Velocity section of this chapter.

\section{Grain Size-Porosity Relationship}

A correlation of the grain-size plots and the porosity plots shown in columns 3 and 4 of the physical property pages should not be attempted since it would be necessary to eliminate the cumulative effects on porosity from other variables, such as: (1) degree of compaction, (2) the grain shape and mean grain size of 
the sand and silt fractions, and (3) the amount of each of the sand and silt fractions.

\section{Grain Size-Gamma-Ray Relationship}

A discussion of the comparison of the grain-size plots with the gamma-ray plots (columns 1 and 3 of the physical property pages, Site Reports, this volume) can be found in the Natural Gamma Radiation section of this chapter.

\section{POROSITY AND WATER CONTENT}

\section{Introduction}

Shipboard measurements of porosity and water content were made on individual small sediment samples. Porosity was also derived from shore based computer processing of the digitized analog produced by the shipboard operation of the GRAPE equipment. A discussion of GRAPE can be found in the Wet-Bulk Density section of this chapter. Figure 7 graphically shows the distribution of these data throughout all of the Leg 11 core holes. Table 4 lists these data for each section of analyzed core.

\section{Shipboard Calculations}

Shipboard calculations of porosity and water content were based on the parameters and equations shown below. No corrections were made for salt content in determination of water content and porosity. According to Hamilton (1971, p. 280):

"porosity in the usual laboratory computations is equal to the weight of evaporated water divided by the volume of saturated sediment; the resulting value is usually listed without correction for the dried salts weighed with the mineral residue. This correction amounts to an increment to porosity, varying according to porosity, from 0.5 to 1 per cent."

A porosity increment of 0.5 to 1 per cent amounts to about 1 to 2 per cent error, or less than the error introduced from other sources.

$$
\begin{gathered}
\begin{aligned}
\mathrm{V} & =\text { Volume of wet sample. } \\
\mathrm{W}_{1}= & \text { Weight of wet sample. } \\
\mathrm{W}_{2}= & \text { Weight of dry sample. } \\
\rho \mathrm{f}= & \begin{array}{l}
\text { Density of pore fluid lost } \\
\text { during drying process }
\end{array} \\
& (1.000 \mathrm{~g} / \mathrm{cc}) .
\end{aligned} \\
\text { Water content }(\%)=\frac{\mathrm{W}_{1}-\mathrm{W}_{2}}{\mathrm{~W}_{1}} \times 100 .
\end{gathered}
$$

Volume of pore space $(\mathrm{cc})=\left(\mathrm{W}_{1}-\mathrm{W}_{2}\right) \div \rho \mathrm{f}$

$$
\text { Porosity }(\%)=\frac{\left(\mathrm{W}_{1}-\mathrm{W}_{2}\right) \div \rho f}{\mathrm{~V}} \times 100
$$

The small (1 to $2 \mathrm{cc}$ ) porosity sample was taken from near the top of each section by inserting a small calibrated plastic syringe into the soft sediment. The sample was treated in the following manner:

1. Known volume of wet sample placed in preweighed receptacle.

2. Weighed wet sample.

3. Sample placed in oven at $105^{\circ} \mathrm{C}$ for 24 hours.

4. Sample cooled at room temperature in dessicator (about one hour).

5. Weighed dried sample.

Porosity errors may be caused by inaccuracies in the volumetric measurements of the sediment sample due to unseen air or gas bubbles. An error of this type would probably cause the calculated porosity to be about 5 per cent less than the true porosity value.

\section{GRAPE Porosity}

The calculation of porosity from GRAPE wet-bulk density is dependent on the relationship of grain density and pore fluid density according to the equation: Wet-bulk density $=$ porosity $\times$ (pore fluid density) + (1-porosity) $\times$ (grain density). A continuous shore-based computer derived porosity plot based on wet-bulk density from the GRAPE analog and grain density supplied by the geologist appears in column 4 of the physical property pages in the Site Reports of this volume. Water content data developed by the shipboard chemist also appear in the same column. A continuous computer plot of the GRAPE analog is shown in column 5. A comparison of the GRAPE porosity plot in column 4 with the GRAPE wet-bulk density plot in column 5 vividly illustrates the fact that these two physical property parameters are inversely proportional.

In order to properly evaluate the accuracy of the porosity values based on the wet-bulk density from the GRAPE analog, it is important to understand the type and magnitude of the errors which could be introduced into the GRAPE measurements. Since the GRAPE device was calibrated to measure a full diameter core liner, the most common error occurs when the device measures a segment of core liner which contains significant concentrations of water, gas, or air in addition to the actual sediment. Drilling water and 


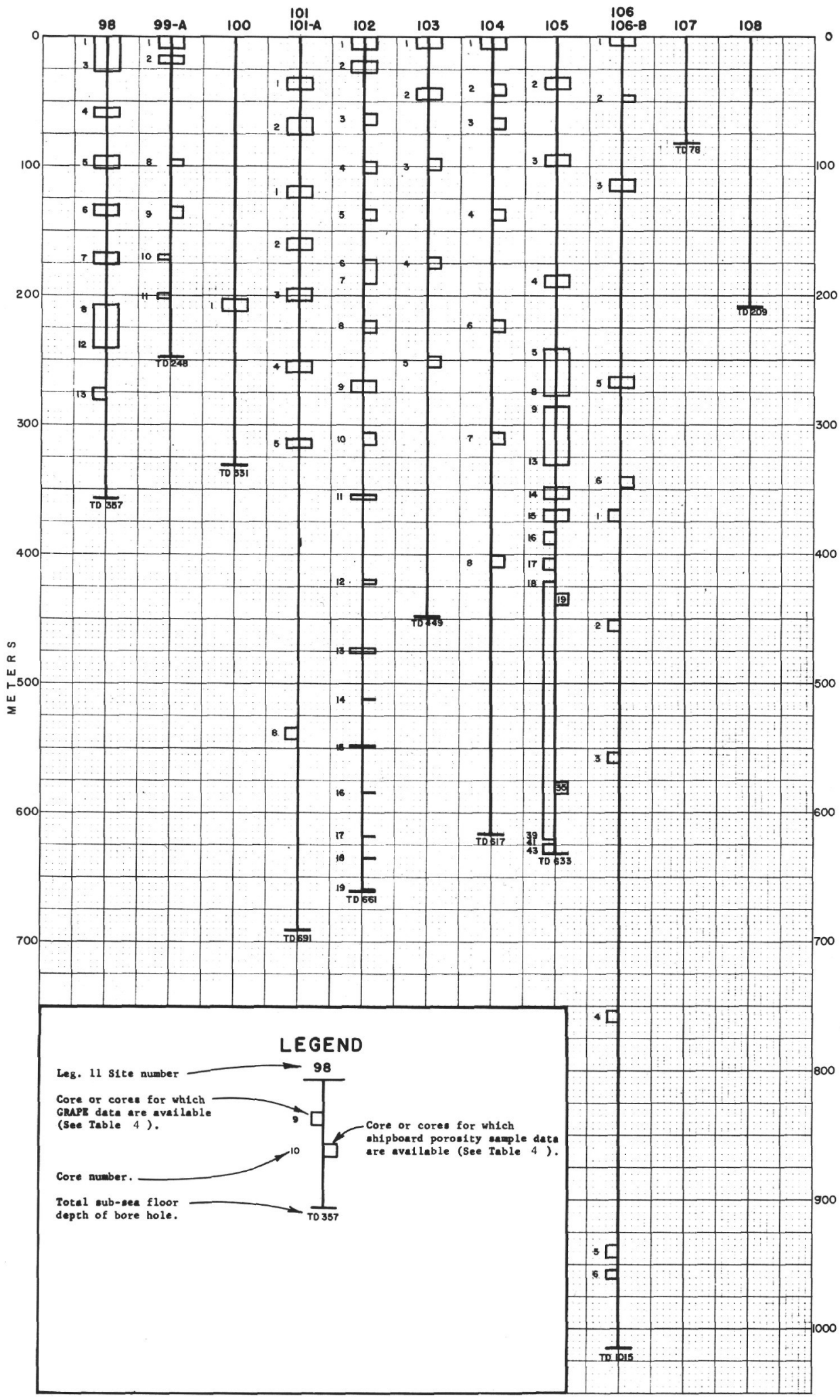

Figure 7. Graphic distribution of GRAPE data and shipboard porosity sample data for all Leg 11 Sites. Only the cored intervals and corresponding core numbers for which data are available have been plotted. Cores with GRAPE data are plotted on the left side of the bore hole and those with porosity sample data are on the right side. Core numbers appear to the left of each bore hole. For continuously cored intervals only the top and bottom core numbers are shown. 
TABLE 4

Wet-Bulk Density, Porosity, Water Content for Leg 11 Sediments

\begin{tabular}{|c|c|c|c|c|c|c|}
\hline \multirow[b]{2}{*}{ Site-Core-Sectiona } & \multirow{2}{*}{$\begin{array}{l}\text { Depthb } \\
\text { (m) }\end{array}$} & \multicolumn{2}{|c|}{$\begin{array}{l}\text { Wet-Bulk Density } \\
\text { (g/cc) }\end{array}$} & \multicolumn{2}{|c|}{$\begin{array}{l}\text { Porosity } \\
\text { (\% vol.) }\end{array}$} & \multirow{2}{*}{$\begin{array}{c}\text { Water } \\
\text { Content } \\
\text { (\% wt.) } \\
\text { Sampled }\end{array}$} \\
\hline & & Grape $^{c}$ & Sample ${ }^{d}$ & Grape $^{c}$ & Sample ${ }^{d}$ & \\
\hline $98-1-1$ & 1 & 1.56 & 1.66 & 68 & 62 & 37 \\
\hline $98-1-2$ & 2 & 1.59 & 1.46 & 66 & 61 & 42 \\
\hline $98-1-3$ & 4 & 1.64 & 1.86 & 64 & 45 & 24 \\
\hline $98-1-4$ & 5 & 1.63 & 1.77 & 64 & 45 & 25 \\
\hline $98-1-5$ & 7 & 1.61 & 1.42 & 66 & 60 & 42 \\
\hline $98-1-6$ & 8 & 1.69 & 1.53 & 60 & 46 & 30 \\
\hline Average & 4 & 1.62 & 1.62 & 65 & 53 & 34 \\
\hline $98-2-1$ & 10 & 1.68 & 1.56 & 61 & 58 & 37 \\
\hline $98-2-2$ & 11 & 1.73 & 1.57 & 58 & 54 & 34 \\
\hline $98-2-3$ & 13 & 1.68 & 1.62 & 61 & 58 & 36 \\
\hline $98-2-4$ & 14 & 1.74 & 1.60 & 57 & 56 & 35 \\
\hline $98-2-5$ & 16 & 1.69 & 1.64 & 60 & 54 & 33 \\
\hline $98-2-6$ & 17 & 1.69 & 1.58 & 61 & 58 & 37 \\
\hline Average & 13 & 1.70 & 1.60 & 60 & 56 & 35 \\
\hline $98-3-1$ & 19 & 1.69 & 1.64 & 60 & 55 & 33 \\
\hline $98-3-2$ & 20 & 1.63 & 1.66 & 64 & 53 & 32 \\
\hline $98-3-3$ & 22 & 1.67 & 1.77 & 61 & 58 & 33 \\
\hline $98-3-4$ & 23 & 1.67 & 1.63 & 62 & 53 & 33 \\
\hline $98-3-5$ & 25 & 1.68 & 1.55 & 61 & 58 & 37 \\
\hline $98-3-6$ & 26 & 1.71 & 1.71 & 59 & 54 & 32 \\
\hline Average & 22 & 1.67 & 1.66 & 61 & 55 & 33 \\
\hline $98-4-1$ & 56 & 1.76 & 1.65 & 56 & 52 & 32 \\
\hline $98-4-2$ & 57 & 1.75 & 1.74 & 57 & 55 & 32 \\
\hline $98-4-3$ & 59 & 1.74 & 1.67 & 57 & 53 & 32 \\
\hline $98-4-4$ & 60 & 1.75 & 1.72 & 57 & 56 & 33 \\
\hline $98-4-5$ & 62 & 1.74 & 1.69 & 57 & 57 & 34 \\
\hline $98-4-6$ & 63 & 1.70 & 1.72 & 60 & 52 & 30 \\
\hline Average & 59 & 1.74 & 1.70 & 57 & 54 & 32 \\
\hline $98-5-1$ & 92 & 1.41 & 1.46 & 77 & 39 & 27 \\
\hline $98-5-2$ & 93 & 1.46 & 1.51 & 74 & 51 & 34 \\
\hline $98-5-3$ & 95 & 1.66 & 1.63 & 63 & 56 & 34 \\
\hline $98-5-4$ & 96 & 1.69 & 1.71 & 60 & 57 & 34 \\
\hline Average & 94 & 1.55 & 1.58 & 68 & 51 & 32 \\
\hline $98-6-1$ & 131 & 1.69 & 1.48 & 60 & 69 & 47 \\
\hline $98-6-2$ & 132 & 1.60 & 1.71 & 65 & 54 & 32 \\
\hline $98-6-3$ & 134 & 1.58 & 1.76 & 67 & 56 & 32 \\
\hline $98-6-4$ & 135 & 1.69 & 1.66 & 60 & 57 & 34 \\
\hline $98-6-5$ & 137 & 1.72 & 1.67 & 58 & 55 & 33 \\
\hline $98-6-6$ & 138 & 1.72 & 1.67 & 58 & 47 & 28 \\
\hline Average & 134 & 1.66 & 1.66 & 61 & 56 & 34 \\
\hline $98-7-1$ & 168 & 1.65 & 1.74 & 63 & 50 & 28 \\
\hline $98-7-2$ & 169 & 1.58 & 1.72 & 67 & 52 & 30 \\
\hline $98-7-3$ & 171 & 1.63 & 1.73 & 64 & 52 & 30 \\
\hline $98-7-4$ & 172 & 1.78 & - & 55 & - & - \\
\hline $98-7-5$ & 174 & 1.76 & 1.75 & 56 & 55 & 31 \\
\hline $98-7-6$ & 175 & 1.86 & 1.77 & 50 & 52 & 29 \\
\hline Average & 171 & 1.71 & 1.74 & 59 & 52 & 30 \\
\hline
\end{tabular}


TABLE 4 - Continued

\begin{tabular}{|c|c|c|c|c|c|c|}
\hline \multirow[b]{2}{*}{ Site-Core-Section ${ }^{\mathrm{a}}$} & \multirow{2}{*}{$\begin{array}{l}\text { Depthb }^{b} \\
(\mathrm{~m})\end{array}$} & \multicolumn{2}{|c|}{$\begin{array}{l}\text { Wet-Bulk Density } \\
\qquad(\mathrm{g} / \mathrm{cc})\end{array}$} & \multicolumn{2}{|c|}{$\begin{array}{l}\text { Porosity } \\
\text { (\% vol.) }\end{array}$} & \multirow{2}{*}{$\begin{array}{c}\text { Water } \\
\text { Contents } \\
\text { (\% wt.) } \\
\text { Sample }^{\mathrm{d}}\end{array}$} \\
\hline & & Grapec & Sample ${ }^{\mathrm{d}}$ & Grape $^{c}$ & Sample ${ }^{\mathrm{d}}$ & \\
\hline $98-8-1$ & 208 & 1.52 & 1.55 & 69 & 59 & 38 \\
\hline $98-8-2$ & 209 & 1.62 & 1.69 & 63 & 56 & 33 \\
\hline $98-8-3$ & 211 & 1.59 & 1.61 & 65 & 54 & 33 \\
\hline Average & 209 & 1.57 & 1.61 & 66 & 56 & 35 \\
\hline 98-9-1 & 217 & 1.48 & 1.54 & 72 & 61 & 39 \\
\hline $98-9-2$ & 218 & 1.69 & 1.76 & 59 & 50 & 28 \\
\hline Average & 217 & 1.58 & 1.65 & 65 & 55 & 34 \\
\hline $98-10-1$ & 223 & 1.57 & 1.86 & 63 & 44 & 24 \\
\hline $98-11-1$ & 232 & 1.69 & 1.84 & 59 & 47 & 25 \\
\hline $98-11-2$ & 233 & 1.85 & 1.66 & 49 & 41 & 25 \\
\hline Average & 232 & 1.76 & 1.75 & 54 & 44 & 25 \\
\hline$* 98-12-1$ & 241 & 1.80 & 1.91 & 53 & 39 & 20 \\
\hline *98-13-1 & 273 & 1.34 & - & 81 & - & - \\
\hline $98-13-2$ & 274 & 1.73 & - & 58 & - & - \\
\hline Average & 273 & 1.53 & - & 69 & - & - \\
\hline $99 A-1-1$ & 1 & 1.47 & 1.51 & 74 & 65 & 43 \\
\hline $99 \mathrm{~A}-1-2$ & 2 & 1.50 & 1.44 & 72 & 64 & 45 \\
\hline $99 A-1-3$ & 4 & 1.48 & 1.41 & 73 & 72 & 51 \\
\hline $99 \mathrm{~A}-1-4$ & 5 & 1.45 & 1.35 & 75 & 69 & 51 \\
\hline $99 A-1-5$ & 7 & 1.41 & - & 78 & - & - \\
\hline Average & 4 & 1.46 & 1.43 & 74 & 68 & 48 \\
\hline $99 A-2-1$ & 16 & 1.31 & 1.36 & 83 & 72 & 53 \\
\hline $99 A-2-2$ & 17 & 1.45 & 1.40 & 75 & 71 & 51 \\
\hline $99 A-2-3$ & 19 & 1.45 & 1.39 & 75 & 71 & 51 \\
\hline $99 A-2-4$ & 20 & 1.45 & 1.40 & 75 & 68 & 49 \\
\hline $99 A-2-5$ & 22 & 1.43 & 1.49 & 76 & 75 & 50 \\
\hline $99 A-2-6$ & 23 & 1.35 & 1.43 & 81 & 69 & 48 \\
\hline Average & 19 & 1.41 & 1.41 & 77 & 71 & 50 \\
\hline 99A-8-1 & 95 & - & 1.83 & - & 42 & 23 \\
\hline 99A-9-1 & 132 & - & 1.94 & - & 41 & 21 \\
\hline 99A-9-6 & 140 & - & 1.85 & - & 44 & 24 \\
\hline Average & 136 & - & 1.89 & - & 42 & 22 \\
\hline $99 \mathrm{~A}-10-1$ & 169 & 1.96 & - & 47 & - & - \\
\hline $99 A-10-2$ & 170 & 2.13 & - & 37 & - & - \\
\hline Average & 169 & 2.04 & - & 42 & - & - \\
\hline
\end{tabular}

*NOTE: The following core analysis data were provided through the courtesy of Core Laboratories, Inc., Dallas, Texas:

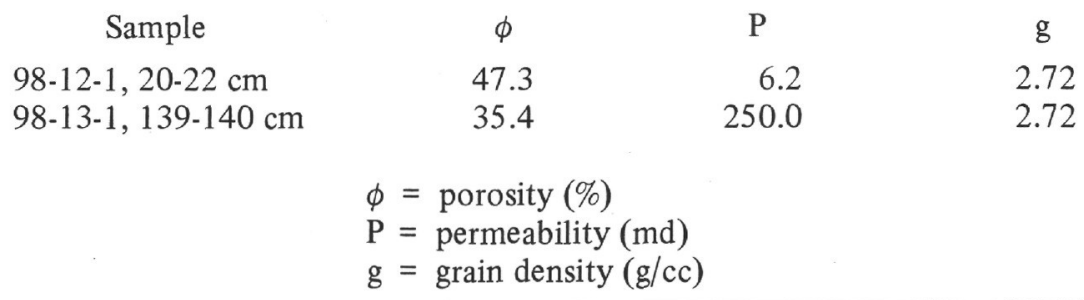


TABLE 4 - Continued

\begin{tabular}{|c|c|c|c|c|c|c|}
\hline \multirow[b]{2}{*}{ Site-Core-Section ${ }^{\mathrm{a}}$} & \multirow{2}{*}{$\begin{array}{l}\text { Depth }^{\mathrm{b}} \\
\text { (m) }\end{array}$} & \multicolumn{2}{|c|}{$\begin{array}{l}\text { Wet-Bulk Density } \\
\qquad(\mathrm{g} / \mathrm{cc})\end{array}$} & \multicolumn{2}{|c|}{$\begin{array}{l}\text { Porosity } \\
\text { (\% vol.) }\end{array}$} & \multirow{2}{*}{$\begin{array}{c}\text { Water } \\
\text { Content } \\
\text { (\% wt.) } \\
\text { Sampled }\end{array}$} \\
\hline & & Grape ${ }^{c}$ & Sample ${ }^{d}$ & Grape $^{c}$ & Sample ${ }^{\mathrm{d}}$ & \\
\hline $99 \mathrm{~A}-11-2$ & 200 & 1.86 & - & 53 & - & - \\
\hline $99 A-11-3$ & 202 & 1.78 & - & 57 & - & - \\
\hline Average & 201 & 1.82 & - & 55 & - & - \\
\hline 101-1-1 & 204 & 1.83 & 1.92 & 53 & 50 & 26 \\
\hline $101-1-2$ & 205 & 1.92 & 2.01 & 48 & 46 & 23 \\
\hline $101-1-3$ & 207 & 1.92 & 1.84 & 48 & 46 & 25 \\
\hline $100-1-4$ & 208 & 1.94 & 1.96 & 47 & 41 & 21 \\
\hline $100-1-5$ & 210 & 1.88 & 1.84 & 50 & 46 & 25 \\
\hline Average & 207 & 1.90 & 1.91 & 49 & 46 & 24 \\
\hline $101-1-1$ & 33 & 1.48 & 1.47 & 78 & 71 & 49 \\
\hline $101-1-2$ & 34 & 1.46 & 1.44 & 78 & 66 & 46 \\
\hline $101-1-3$ & 36 & 1.50 & 1.49 & 76 & 66 & 44 \\
\hline $101-1-4$ & 37 & 1.50 & 1.46 & 76 & 63 & 43 \\
\hline $101-1-5$ & 39 & 1.51 & 1.39 & 75 & 59 & 42 \\
\hline $101-1-6$ & 40 & 1.47 & 1.46 & 77 & 64 & 43 \\
\hline Average & 37 & 1.49 & 1.45 & 76 & 65 & 45 \\
\hline $101-2-1$ & 68 & 1.39 & 1.36 & 82 & 73 & 54 \\
\hline $101-2-2$ & 69 & 1.41 & 1.29 & 81 & 65 & 50 \\
\hline $101-2-3$ & 71 & 1.57 & 1.54 & 73 & 64 & 42 \\
\hline $101-2-4$ & 72 & 1.57 & 1.65 & 73 & 63 & 38 \\
\hline $101-2-5$ & 74 & 1.60 & 1.56 & 71 & 59 & 38 \\
\hline Average & 71 & 1.51 & 1.48 & 76 & 65 & 44 \\
\hline $101 \mathrm{~A}-1-1$ & 116 & 1.59 & 1.49 & 72 & 61 & 41 \\
\hline $101 \mathrm{~A}-1-2$ & 117 & 1.64 & 1.60 & 70 & 56 & 35 \\
\hline $101 \mathrm{~A}-1-3$ & 119 & 1.56 & 1.67 & 74 & 55 & 33 \\
\hline Average & 117 & 1.60 & 1.59 & 72 & 57 & 36 \\
\hline $101 \mathrm{~A}-2-1$ & 157 & 1.59 & 1.50 & 70 & 60 & 40 \\
\hline $101 \mathrm{~A}-2-2$ & 158 & 1.65 & 1.68 & 67 & 62 & 37 \\
\hline $101 \mathrm{~A}-2-3$ & 160 & 1.68 & 1.68 & 66 & 56 & 33 \\
\hline $101 \mathrm{~A}-2-4$ & 161 & 1.68 & 1.58 & 66 & 52 & 33 \\
\hline $101 \mathrm{~A}-2-5$ & 163 & 1.66 & 1.65 & 67 & 54 & 33 \\
\hline $101 \mathrm{~A}-2-6$ & 164 & 1.70 & 1.61 & 65 & 55 & 34 \\
\hline Average & 160 & 1.66 & 1.62 & 67 & 56 & 35 \\
\hline 101A-3-1 & 195 & - & 1.56 & - & 59 & 38 \\
\hline $101 \mathrm{~A}-3-2$ & 196 & 1.60 & 1.66 & 68 & 55 & 33 \\
\hline Average & 195 & - & 1.61 & - & 57 & 35 \\
\hline $101 \mathrm{~A}-4-1$ & 251 & 1.50 & 1.69 & 72 & 53 & 31 \\
\hline $101 A-5-1$ & 309 & 1.51 & - & 71 & - & - \\
\hline $101 \mathrm{~A}-5-2$ & 310 & 1.51 & 1.50 & 71 & 59 & 39 \\
\hline Average & 309 & 1.51 & - & 71 & - & - \\
\hline $101 \mathrm{~A}-8-1$ & 535 & 1.87 & - & 50 & - & - \\
\hline $101 \mathrm{~A}-8-2$ & 536 & 1.86 & - & 50 & - & - \\
\hline Average & 535 & 1.86 & - & 50 & - & - \\
\hline 102-1-1 & 1 & 1.35 & - & 81 & - & - \\
\hline $102-1-2$ & 2 & 1.36 & 1.41 & 80 & 79 & 56 \\
\hline $102-1-3$ & 4 & 1.37 & 1.45 & 80 & 70 & 48 \\
\hline $102-1-4$ & 5 & 1.41 & 1.45 & 77 & 72 & 50 \\
\hline
\end{tabular}


TABLE 4 - Continued

\begin{tabular}{|c|c|c|c|c|c|c|}
\hline \multirow[b]{2}{*}{ Site-Core-Section ${ }^{\mathrm{a}}$} & \multirow{2}{*}{$\begin{array}{l}\text { Depthb }^{b} \\
\text { (m) }\end{array}$} & \multicolumn{2}{|c|}{$\begin{array}{l}\text { Wet-Bulk Density } \\
\text { (g/cc) }\end{array}$} & \multicolumn{2}{|c|}{$\begin{array}{l}\text { Porosity } \\
\text { (\% vol.) }\end{array}$} & \multirow{2}{*}{$\begin{array}{c}\text { Water } \\
\text { Content } \\
\text { (\% wt.) } \\
\text { Sample }^{\mathrm{d}}\end{array}$} \\
\hline & & Grape ${ }^{c}$ & Sample & Grape $^{c}$ & Sample & \\
\hline $102-1-5$ & 7 & 1.41 & 1.40 & 77 & 69 & 49 \\
\hline $102-1-6$ & 8 & 1.44 & - & 75 & - & - \\
\hline Average & 4 & 1.39 & 1.43 & 78 & 72 & 51 \\
\hline $102-2-1$ & 19 & 1.33 & - & 82 & - & - \\
\hline $102-2-2$ & 20 & 1.34 & 1.44 & 82 & 75 & 52 \\
\hline $102-2-3$ & 21 & - & 1.42 & - & 72 & 51 \\
\hline $102-2-4$ & 23 & - & 1.38 & - & 66 & 48 \\
\hline $102-2-5$ & 24 & - & 1.42 & - & 63 & 45 \\
\hline $102-2-6$ & 26 & - & 1.38 & - & 72 & 52 \\
\hline Average & 21 & 1.34 & 1.41 & 82 & 70 & 49 \\
\hline $102-3-1$ & 59 & - & 1.44 & - & 68 & 47 \\
\hline $102-3-2$ & 60 & - & 1.49 & - & 66 & 44 \\
\hline Average & 59 & - & 1.46 & - & 67 & 46 \\
\hline $102-4-3$ & 99 & - & 1.72 & - & 56 & 32 \\
\hline $102-4-4$ & 101 & - & 1.72 & - & 58 & 34 \\
\hline Average & 100 & - & 1.72 & - & 57 & 33 \\
\hline $102-5-1$ & 133 & - & 1.54 & - & 59 & 38 \\
\hline $102-5-2$ & 135 & - & 1.58 & - & 55 & 35 \\
\hline $102-5-3$ & 136 & - & 1.43 & - & 51 & 36 \\
\hline $102-5-4$ & 138 & - & 1.76 & - & 53 & 30 \\
\hline $102-5-5$ & 139 & - & 1.62 & - & 49 & 30 \\
\hline $102-5-6$ & 141 & - & 1.63 & - & 52 & 32 \\
\hline Average & 137 & - & 1.59 & - & 53 & 34 \\
\hline $102-6-1$ & 172 & - & 1.43 & - & 50 & 35 \\
\hline $102-6-2$ & 174 & - & 1.77 & - & 42 & 23 \\
\hline $102-6-4$ & 177 & - & 1.41 & - & 53 & 38 \\
\hline $102-6-5$ & 178 & - & 1.50 & - & 62 & 41 \\
\hline $102-6-6$ & 180 & - & 1.53 & - & 62 & 41 \\
\hline Average & 176 & - & 1.53 & - & 54 & 36 \\
\hline $102-7-1$ & 181 & - & 1.55 & - & 58 & 37 \\
\hline $102-7-2$ & 183 & - & 1.52 & - & 58 & 38 \\
\hline $102-7-3$ & 184 & - & 1.54 & - & 53 & 34 \\
\hline $102-7-4$ & 186 & - & 1.89 & - & 44 & 23 \\
\hline $102-7-5$ & 187 & - & 1.38 & - & 47 & 34 \\
\hline $102-7-6$ & 189 & - & 1.43 & - & 46 & 32 \\
\hline Average & 185 & - & 1.55 & - & 51 & 33 \\
\hline $102-8-1$ & 219 & - & 1.53 & - & 58 & 38 \\
\hline $102-8-2$ & 221 & - & 1.58 & - & 62 & 39 \\
\hline $102-8-3$ & 223 & - & 1.55 & - & 56 & 36 \\
\hline $102-8-4$ & 224 & - & 1.58 & - & 61 & 38 \\
\hline $102-8-5$ & 225 & - & 1.49 & - & 59 & 39 \\
\hline $102-8-6$ & 227 & - & 1.58 & - & 59 & 37 \\
\hline Average & 223 & - & 1.55 & - & 59 & 38 \\
\hline 102-9-1 & 267 & 1.68 & 1.71 & 64 & 53 & 31 \\
\hline $102-9-2$ & 268 & 1.69 & 1.70 & 64 & 50 & 29 \\
\hline $102-9-3$ & 269 & - & 1.71 & - & 51 & 30 \\
\hline $102-9-4$ & 271 & - & 1.70 & - & 53 & 31 \\
\hline $102-9-5$ & 272 & - & 1.68 & - & 47 & 28 \\
\hline
\end{tabular}


TABLE 4 - Continued

\begin{tabular}{|c|c|c|c|c|c|c|}
\hline \multirow[b]{2}{*}{ Site-Core-Section ${ }^{a}$} & \multirow{2}{*}{$\begin{array}{l}\text { Depth }^{b} \\
\text { (m) }\end{array}$} & \multicolumn{2}{|c|}{$\begin{array}{l}\text { Wet-Bulk Density } \\
\qquad(\mathrm{g} / \mathrm{cc})\end{array}$} & \multicolumn{2}{|c|}{$\begin{array}{l}\text { Porosity } \\
\text { (\% vol.) }\end{array}$} & \multirow{2}{*}{$\begin{array}{c}\text { Water } \\
\text { Content } \\
\text { (\% wt.) } \\
\text { Sampled }\end{array}$} \\
\hline & & Grape $^{c}$ & Sample ${ }^{d}$ & Grape $^{c}$ & Sample ${ }^{d}$ & \\
\hline Average & 269 & 1.68 & 1.70 & 64 & 51 & 30 \\
\hline $102-10-1$ & 307 & - & 1.51 & - & 46 & 30 \\
\hline $102-10-2$ & 308 & - & 1.64 & - & 53 & 32 \\
\hline $102-10-3$ & 309 & - & 1.65 & - & 57 & 34 \\
\hline $102-10-4$ & 311 & - & 1.57 & - & 55 & 35 \\
\hline $102-10-5$ & 312 & - & 1.57 & - & 45 & 29 \\
\hline $102-10-6$ & 314 & - & 1.63 & - & 52 & 32 \\
\hline Average & 311 & - & 1.59 & - & 51 & 32 \\
\hline $102-11-1$ & 353 & - & 1.57 & - & 53 & 33 \\
\hline $102-11-2$ & 354 & - & 1.60 & - & 46 & 28 \\
\hline $102-11-3$ & 357 & 1.60 & 1.39 & 69 & 40 & 29 \\
\hline Average & 355 & - & 1.52 & - & 46 & 30 \\
\hline $102-12-1$ & 419 & - & 1.73 & - & 53 & 30 \\
\hline $102-12-2$ & 421 & - & 1.77 & - & 49 & 27 \\
\hline $102-12-3$ & 422 & - & 1.66 & - & 52 & 32 \\
\hline $102-12-4$ & 424 & - & 1.69 & - & 48 & 29 \\
\hline Average & 421 & - & 1.71 & - & 50 & 20 \\
\hline $102-13-1$ & 473 & - & 1.68 & - & 50 & 30 \\
\hline $102-13-2$ & 475 & - & 1.78 & - & 45 & 25 \\
\hline $102-13-3$ & 477 & - & 1.66 & - & 46 & 28 \\
\hline $102-13-4$ & 478 & - & 1.80 & - & 43 & 24 \\
\hline Average & 476 & - & 1.73 & - & 46 & 27 \\
\hline $102-14-1$ & 512 & - & 1.67 & - & 47 & 28 \\
\hline $102-15-1$ & 549 & 1.62 & 1.58 & 69 & 40 & 25 \\
\hline $102-16-1$ & 584 & - & 1.55 & - & 43 & 28 \\
\hline $102-17-1$ & 618 & - & 1.67 & - & 49 & 29 \\
\hline $102-18-1$ & 635 & - & 1.63 & - & 45 & 28 \\
\hline $102-18-2$ & 636 & - & 1.67 & - & 40 & 24 \\
\hline $102-18-3$ & 637 & - & 1.81 & - & 41 & 22 \\
\hline $102-18-4$ & 639 & - & 1.77 & - & 41 & 23 \\
\hline Average & 637 & - & 1.72 & - & 42 & 24 \\
\hline $102-19-1$ & 660 & - & 1.66 & - & 45 & 27 \\
\hline $103-1-1$ & 1 & 1.50 & 1.33 & 74 & 49 & 37 \\
\hline $103-1-2$ & 2 & 1.51 & 1.45 & 73 & 64 & 44 \\
\hline $103-1-3$ & 4 & 1.47 & 1.36 & 75 & 67 & 49 \\
\hline $103-1-4$ & 5 & 1.48 & 1.34 & 75 & 68 & 50 \\
\hline $103-1-5$ & 7 & 1.49 & 1.45 & 74 & 67 & 46 \\
\hline $103-1-6$ & 8 & 1.52 & 1.45 & 72 & 66 & 46 \\
\hline Average & 4 & 1.49 & 1.40 & 74 & 63 & 45 \\
\hline $103-2-1$ & 40 & 1.50 & 1.30 & 73 & 60 & 46 \\
\hline $103-2-2$ & 41 & 1.48 & 1.58 & 75 & 68 & 43 \\
\hline $103-2-3$ & 43 & 1.56 & 1.44 & 71 & 64 & 44 \\
\hline $103-2-4$ & 44 & 1.62 & 1.57 & 67 & 60 & 38 \\
\hline $103-2-5$ & 46 & 1.63 & 1.55 & 67 & 59 & 38 \\
\hline $103-2-6$ & 47 & 1.77 & 1.89 & 59 & 40 & 21 \\
\hline Average & 43 & 1.59 & 1.55 & 69 & 58 & 38 \\
\hline 103-3-1 & 94 & - & 1.41 & - & 62 & 44 \\
\hline
\end{tabular}


TABLE 4 - Continued

\begin{tabular}{|c|c|c|c|c|c|c|}
\hline \multirow[b]{2}{*}{ Site-Core-Section ${ }^{\mathrm{a}}$} & \multirow{2}{*}{$\begin{array}{l}\text { Depthb }^{b} \\
(\mathrm{~m})\end{array}$} & \multicolumn{2}{|c|}{$\begin{array}{l}\text { Wet-Bulk Density } \\
\qquad(\mathrm{g} / \mathrm{cc})\end{array}$} & \multicolumn{2}{|c|}{$\begin{array}{l}\text { Porosity } \\
\text { (\% vol.) }\end{array}$} & \multirow{2}{*}{$\begin{array}{c}\text { Water } \\
\text { Content } \\
\text { (\% wt.) } \\
\text { Sample }^{\mathrm{d}}\end{array}$} \\
\hline & & Grape $^{c}$ & Sampled & Grape $^{c}$ & Sampled & \\
\hline $103-3-2$ & 96 & - & 1.44 & - & 59 & 41 \\
\hline $103-3-3$ & 97 & - & 1.37 & - & 57 & 42 \\
\hline $103-3-4$ & 99 & - & 1.52 & - & 62 & 41 \\
\hline $103-3-5$ & 100 & - & 1.52 & - & 64 & 42 \\
\hline $103-3-6$ & 102 & - & 1.52 & - & 60 & 39 \\
\hline Average & 98 & - & 1.46 & - & 60 & 41 \\
\hline $103-4-2$ & 172 & - & 1.43 & - & 76 & 53 \\
\hline $103-4-3$ & 173 & - & 1.42 & - & 62 & 44 \\
\hline $103-4-4$ & 175 & - & 1.46 & - & 55 & 37 \\
\hline $103-4-5$ & 176 & - & 1.52 & - & 58 & 38 \\
\hline Average & 174 & - & 1.46 & - & 63 & 43 \\
\hline $103-5-1$ & 248 & - & 1.57 & - & 53 & 34 \\
\hline $103-5-3$ & 250 & - & 1.69 & - & 47 & 28 \\
\hline Average & 249 & - & 1.63 & - & 50 & 31 \\
\hline 104-1-1 & 1 & 1.48 & 1.49 & 74 & 66 & 44 \\
\hline $104-1-2$ & 2 & 1.45 & 1.31 & 76 & 66 & 50 \\
\hline $104-1-3$ & 4 & 1.52 & 1.43 & 72 & 77 & 54 \\
\hline $104-1-4$ & 5 & 1.52 & 1.46 & 72 & 69 & 47 \\
\hline $104-1-5$ & 7 & 1.45 & 1.40 & 76 & 70 & 50 \\
\hline 104-1-6 & 8 & 1.43 & 1.39 & 77 & 70 & 50 \\
\hline Average & 4 & 1.51 & 1.41 & 74 & 70 & 49 \\
\hline 104-2-1 & 37 & - & 1.42 & - & 67 & 47 \\
\hline $104-2-2$ & 38 & - & 1.49 & - & 64 & 43 \\
\hline $104-2-3$ & 39 & - & 1.59 & - & 68 & 43 \\
\hline $104-2-4$ & 41 & - & 1.58 & - & 64 & 40 \\
\hline $104-2-5$ & 42 & - & 1.47 & - & 60 & 40 \\
\hline $104-2-6$ & 44 & - & 1.58 & - & 61 & 39 \\
\hline Average & 40 & - & 1.52 & - & 64 & 42 \\
\hline 104-3-1 & 62 & - & 1.37 & - & 74 & 54 \\
\hline $104-3-2$ & 64 & - & 1.29 & - & 71 & 55 \\
\hline $104-3-3$ & 65 & - & 1.29 & - & 74 & 57 \\
\hline $104-3-4$ & 67 & - & 1.16 & - & 66 & 57 \\
\hline 104-3-6 & 70 & - & 1.26 & - & 69 & 55 \\
\hline Average & 66 & - & 1.27 & - & 71 & 56 \\
\hline 104-4-1 & 133 & - & 1.35 & - & 57 & 42 \\
\hline $104-4-3$ & 136 & - & 1.44 & - & 67 & 47 \\
\hline $104-4-4$ & 138 & - & 1.43 & - & 67 & 47 \\
\hline $104-4-5$ & 139 & - & 1.42 & - & 67 & 47 \\
\hline 104-4-6 & 140 & - & 1.39 & - & 66 & 48 \\
\hline Average & 137 & - & 1.41 & - & 65 & 46 \\
\hline 104-6-1 & 219 & - & 1.34 & - & 62 & 46 \\
\hline $104-6-2$ & 221 & - & 1.45 & - & 70 & 48 \\
\hline $104-6-3$ & 222 & - & 1.34 & - & 62 & 47 \\
\hline $104-6-4$ & 224 & - & 1.37 & - & 62 & 45 \\
\hline $104-6-5$ & 225 & - & 1.41 & - & 66 & 47 \\
\hline 104-6-6 & 227 & - & 1.40 & - & 61 & 44 \\
\hline Average & 223 & - & 1.38 & - & 64 & 46 \\
\hline $104-7-1$ & 306 & - & 1.45 & - & 64 & 44 \\
\hline
\end{tabular}


TABLE 4 - Continued

\begin{tabular}{|c|c|c|c|c|c|c|}
\hline \multirow[b]{2}{*}{ Site-Core-Section ${ }^{\mathrm{a}}$} & \multirow{2}{*}{$\begin{array}{l}\text { Depth }^{\mathrm{b}} \\
(\mathrm{m})\end{array}$} & \multicolumn{2}{|c|}{$\begin{array}{l}\text { Wet-Bulk Density } \\
(\mathrm{g} / \mathrm{cc})\end{array}$} & \multicolumn{2}{|c|}{$\begin{array}{l}\text { Porosity } \\
\text { (\% vol.) }\end{array}$} & \multirow{2}{*}{$\begin{array}{c}\text { Water } \\
\text { Content } \\
\text { (\% wt.) } \\
\text { Sample }^{\mathrm{d}}\end{array}$} \\
\hline & & $\mathrm{Grape}^{\mathrm{c}}$ & Sample $\mathrm{d}^{\mathrm{d}}$ & Grape $^{c}$ & Sampled & \\
\hline $104-7-2$ & 308 & - & 1.40 & - & 59 & 42 \\
\hline $104-7-3$ & 309 & - & 1.53 & - & 62 & 40 \\
\hline $104-7-4$ & 311 & - & 1.52 & - & 63 & 41 \\
\hline Average & 308 & - & 1.48 & - & 62 & 42 \\
\hline 104-8-1 & 402 & - & 1.65 & - & 64 & 39 \\
\hline $104-8-2$ & 403 & - & 1.22 & - & 46 & 38 \\
\hline $104-8-3$ & 404 & - & 1.20 & - & 48 & 40 \\
\hline $104-8-4$ & 406 & - & 1.41 & - & 56 & 39 \\
\hline $104-8-5$ & 407 & - & 1.29 & - & 50 & 39 \\
\hline Average & 404 & - & 1.35 & - & 53 & 39 \\
\hline $105-2-1$ & 32 & 1.74 & 1.56 & 60 & 55 & 35 \\
\hline $105-2-2$ & 33 & 1.66 & 1.83 & 65 & 57 & 31 \\
\hline $105-2-3$ & 35 & 1.79 & 1.69 & 57 & 59 & 35 \\
\hline Average & 33 & 1.73 & 1.69 & 61 & 57 & 34 \\
\hline $103-3-1$ & 92 & 1.72 & 1.59 & 61 & 56 & 35 \\
\hline $105-3-2$ & 93 & 1.66 & 1.64 & 64 & 63 & 38 \\
\hline $105-3-3$ & 95 & 1.67 & 1.63 & 64 & 56 & 34 \\
\hline $105-3-4$ & 96 & 1.75 & 1.63 & 59 & 55 & 33 \\
\hline Average & 94 & 1.70 & 1.62 & 62 & 57 & 35 \\
\hline $105-4-1$ & 185 & 1.58 & 1.57 & 61 & 63 & 40 \\
\hline $105-4-2$ & 186 & 1.74 & 1.56 & 50 & 34 & 53 \\
\hline $105-4-3$ & 188 & 1.72 & 1.68 & 51 & 55 & 33 \\
\hline $105-4-4$ & 189 & 1.76 & 1.36 & 49 & 46 & 34 \\
\hline $105-4-5$ & 191 & 1.72 & 1.69 & 51 & 52 & 31 \\
\hline $105-4-6$ & 192 & 1.71 & 1.47 & 52 & 42 & 28 \\
\hline Average & 188 & 1.70 & 1.55 & 52 & 49 & 33 \\
\hline $105-5-1$ & 242 & 1.63 & 1.68 & 67 & 61 & 36 \\
\hline $105-5-2$ & 243 & 1.68 & 1.65 & 64 & 56 & 34 \\
\hline $105-5-3$ & 245 & 1.58 & - & 69 & - & - \\
\hline Average & 243 & 1.63 & 1.66 & 67 & 58 & 35 \\
\hline 105-6-1 & 251 & 1.77 & 1.72 & 61 & 52 & 30 \\
\hline $105-6-2$ & 252 & 1.75 & 1.60 & 59 & 45 & 28 \\
\hline Average & 251 & 1.73 & 1.66 & 60 & 48 & 29 \\
\hline $105-7-$ & \multicolumn{5}{|c|}{ (Sections $1-5$ consist of hole cavings) } & \\
\hline $105-7-6$ & 267 & 1.87 & 1.78 & 48 & 47 & 26 \\
\hline $105-8-1$ & 269 & 1.85 & 1.75 & 49 & 52 & 30 \\
\hline $105-8-2$ & 270 & 1.84 & 1.78 & 50 & 48 & 27 \\
\hline $105-8-3$ & 272 & 1.82 & 1.75 & 52 & 54 & 31 \\
\hline $105-8-4$ & 273 & 1.83 & 1.78 & 53 & 27 & 48 \\
\hline \multicolumn{6}{|c|}{ (Sections 5 and 6 contain hole cavings) } & \\
\hline Average & 271 & 1.83 & 1.76 & 51 & 45 & 34 \\
\hline 105-9-1 & 287 & 1.81 & 1.89 & 56 & 43 & 23 \\
\hline $105-9-2$ & 288 & 1.76 & 1.71 & 58 & 44 & 26 \\
\hline $105-9-3$ & 290 & 1.69 & 1.72 & 62 & 48 & 28 \\
\hline $105-9-4$ & 291 & 1.58 & - & 68 & - & - \\
\hline $105-9-5$ & 293 & 1.69 & - & 62 & - & - \\
\hline $105-9-6$ & 294 & 1.70 & - & 55 & - & - \\
\hline Average & 290 & 1.70 & 1.77 & 60 & 45 & 25 \\
\hline
\end{tabular}


TABLE 4 - Continued

\begin{tabular}{|c|c|c|c|c|c|c|}
\hline \multirow[b]{2}{*}{ Site-Core-Section ${ }^{\mathrm{a}}$} & \multirow{2}{*}{$\begin{array}{l}\text { Depth }^{b} \\
(\mathrm{~m})\end{array}$} & \multicolumn{2}{|c|}{$\begin{array}{l}\text { Wet-Bulk Density } \\
\qquad(\mathrm{g} / \mathrm{cc})\end{array}$} & \multicolumn{2}{|c|}{$\begin{array}{l}\text { Porosity } \\
\text { (\% vol.) }\end{array}$} & \multirow{2}{*}{$\begin{array}{c}\text { Water } \\
\text { Content } \\
\text { (\% wt.) } \\
\text { Sample }\end{array}$} \\
\hline & & Grape $^{c}$ & Sample ${ }^{\mathrm{d}}$ & Grape ${ }^{c}$ & Sample ${ }^{\mathrm{d}}$ & \\
\hline $105-10-1$ & 296 & 1.68 & 1.51 & 56 & 63 & 42 \\
\hline $105-10-2$ & 297 & 1.75 & - & 51 & - & - \\
\hline Average & 296 & 1.71 & - & 53 & - & - \\
\hline $105-11-1$ & 305 & 1.68 & - & 57 & - & - \\
\hline $105-11-2$ & 306 & 1.69 & - & 56 & - & - \\
\hline $105-11-3$ & 308 & 1.67 & - & 57 & - & - \\
\hline $105-11-4$ & 309 & 1.69 & - & 56 & - & - \\
\hline $105-11-5$ & 311 & 1.67 & - & 57 & - & - \\
\hline Average & 308 & 1.68 & - & 57 & - & - \\
\hline $105-12-1$ & 314 & 1.65 & 1.49 & 58 & 54 & 36 \\
\hline $105-12-2$ & 315 & 1.75 & 1.59 & 52 & 45 & 28 \\
\hline $105-12-3$ & 317 & 1.70 & 1.74 & 55 & 54 & 31 \\
\hline $105-12-4$ & 318 & 1.70 & 1.84 & 56 & 57 & 31 \\
\hline Average & 316 & 1.70 & 1.67 & 55 & 52 & 31 \\
\hline \multicolumn{7}{|c|}{$\begin{array}{l}\text { (Sections } 1-4 \text { consist of hole cavings) } \\
\text { (Sections } 5 \text { and } 6 \text { are primarily drilling mud) }\end{array}$} \\
\hline $105-14-1$ & 349 & 1.59 & - & 63 & - & - \\
\hline $105-15-1$ & 367 & 1.50 & 1.41 & 71 & 59 & 41 \\
\hline $105-15-2$ & 368 & 1.62 & 1.44 & 64 & 61 & 42 \\
\hline $105-15-3$ & 370 & 1.68 & - & 60 & - & - \\
\hline $105-15-4$ & 371 & 1.68 & - & 60 & - & - \\
\hline $105-15-5$ & 373 & 1.71 & - & 58 & - & - \\
\hline $105-15-6$ & 374 & 1.67 & - & 61 & - & - \\
\hline Average & 370 & 1.56 & 1.43 & 62 & 60 & 42 \\
\hline $105-16-1$ & 386 & 1.46 & - & 74 & - & - \\
\hline $105-16-2$ & 387 & 1.59 & - & 65 & - & - \\
\hline Average & 386 & 1.52 & - & 69 & - & - \\
\hline $105-17-2$ & 405 & 1.39 & - & 79 & - & - \\
\hline $105-17-3$ & 407 & 1.57 & - & 68 & - & - \\
\hline Average & 406 & 1.48 & - & 73 & - & - \\
\hline $105-18-1$ & 422 & 1.53 & - & 71 & - & - \\
\hline $105-18-2$ & 423 & 1.57 & - & 68 & - & - \\
\hline $105-18-3$ & 425 & 1.67 & - & 63 & - & - \\
\hline $105-18-4$ & 426 & 1.65 & - & 64 & - & - \\
\hline $105-18-5$ & 428 & 1.66 & - & 63 & - & - \\
\hline $105-18-6$ & 429 & 1.66 & - & 63 & - & - \\
\hline Average & 425 & 1.62 & - & 65 & - & - \\
\hline 105-19- & \multicolumn{5}{|c|}{ (All four sections consist of hole cavings.) } & \\
\hline $105-20-1$ & 440 & 1.57 & - & 67 & - & - \\
\hline $105-21-1$ & 449 & 1.34 & - & 82 & - & - \\
\hline $105-21-2$ & 450 & 1.47 & - & 74 & - & - \\
\hline Average & 449 & 1.40 & - & 78 & - & - \\
\hline $105-22-1$ & 458 & 1.67 & - & 62 & - & - \\
\hline $105-22-2$ & 459 & 1.57 & - & 68 & - & - \\
\hline Average & 458 & 1.62 & - & 65 & - & - \\
\hline $105-23-1$ & 467 & 1.80 & - & 54 & - & - \\
\hline $105-23-2$ & 468 & 1.97 & - & 44 & - & - \\
\hline
\end{tabular}


TABLE 4 - Continued

\begin{tabular}{|c|c|c|c|c|c|c|}
\hline \multirow[b]{2}{*}{ Site-Core-Section ${ }^{\mathrm{a}}$} & \multirow{2}{*}{$\begin{array}{c}\text { Depthb } \\
(\mathrm{m})\end{array}$} & \multicolumn{2}{|c|}{$\begin{array}{l}\text { Wet-Bulk Density } \\
(\mathrm{g} / \mathrm{cc})\end{array}$} & \multicolumn{2}{|c|}{$\begin{array}{r}\text { Porosity } \\
\text { (\% vol.) }\end{array}$} & \multirow{2}{*}{$\begin{array}{c}\text { Water } \\
\text { Content } \\
(\% \text { wt.) } \\
\text { Sample }^{\mathrm{d}}\end{array}$} \\
\hline & & $\mathrm{Grape}^{\mathrm{c}}$ & Sample ${ }^{\mathrm{d}}$ & Grape $^{c}$ & Sample ${ }^{\mathrm{d}}$ & \\
\hline Average & 467 & 1.88 & - & 49 & - & - \\
\hline $105-24-1$ & 476 & 1.61 & - & 67 & - & - \\
\hline $105-24-2$ & 477 & 1.68 & - & 63 & - & - \\
\hline Average & 476 & 1.64 & - & 65 & - & - \\
\hline $105-25-1$ & 485 & 1.57 & - & 69 & - & - \\
\hline $105-25-2$ & 486 & 1.58 & - & 68 & - & - \\
\hline $105-25-3$ & 487 & 1.65 & - & 64 & - & - \\
\hline Average & 486 & 1.60 & - & 67 & - & - \\
\hline $105-26-1$ & 494 & 1.66 & - & 63 & - & - \\
\hline $105-26-2$ & 495 & 1.87 & - & 51 & - & - \\
\hline Average & 494 & 1.76 & - & 57 & - & - \\
\hline $105-27-1$ & 503 & 1.66 & - & 63 & - & - \\
\hline $105-27-2$ & 504 & 1.60 & - & 66 & - & - \\
\hline $105-27-3$ & 506 & 1.62 & - & 65 & - & - \\
\hline Average & 504 & 1.63 & - & 65 & - & - \\
\hline $105-28-1$ & 512 & 1.68 & - & 63 & - & - \\
\hline $105-28-2$ & 513 & 1.68 & - & 63 & - & - \\
\hline $105-28-3$ & 515 & 1.73 & - & 60 & - & - \\
\hline $105-28-4$ & 516 & 1.69 & - & 62 & - & - \\
\hline $105-28-5$ & 518 & 1.66 & - & 64 & - & - \\
\hline $105-28-6$ & 519 & 1.81 & - & 55 & - & - \\
\hline Average & 515 & 1.71 & - & 61 & - & - \\
\hline $105-29-1$ & 523 & 1.66 & - & 64 & - & - \\
\hline $105-29-2$ & 524 & 1.64 & - & 65 & - & - \\
\hline $105-29-3$ & 526 & 1.66 & - & 64 & - & - \\
\hline Average & 524 & 1.65 & - & 64 & - & - \\
\hline 105-30-1 & 532 & 1.58 & - & 68 & - & - \\
\hline $105-30-2$ & 533 & 1.59 & - & 68 & - & - \\
\hline Average & 532 & 1.58 & - & 68 & - & - \\
\hline $105-31-1$ & 541 & 1.39 & - & 79 & - & - \\
\hline $105-31-2$ & 542 & 1.81 & - & 55 & - & - \\
\hline Average & 541 & 1.61 & - & 67 & - & - \\
\hline $105-32-1$ & 550 & 1.69 & - & 62 & - & - \\
\hline $105-32-2$ & 551 & 1.61 & - & 67 & - & - \\
\hline Average & 550 & 1.65 & - & 64 & - & - \\
\hline $105-33-1$ & 559 & 1.53 & - & 71 & - & - \\
\hline $105-33-2$ & 560 & 1.78 & - & 58 & - & - \\
\hline $105-33-3$ & 562 & 1.79 & - & 57 & - & - \\
\hline $105-33-4$ & 563 & 1.82 & - & 55 & - & - \\
\hline $105-33-5$ & 565 & 1.84 & - & 54 & - & - \\
\hline Average & 562 & 1.75 & - & 59 & - & - \\
\hline $105-34-1$ & 568 & 1.84 & - & 54 & - & - \\
\hline $105-34-2$ & 569 & 1.78 & - & 57 & - & - \\
\hline $105-34-3$ & 571 & 1.79 & - & 57 & - & - \\
\hline $105-34-4$ & 572 & 1.80 & - & 56 & - & - \\
\hline $105-34-5$ & 574 & 1.70 & - & 62 & - & - \\
\hline
\end{tabular}


TABLE 4 - Continued

\begin{tabular}{|c|c|c|c|c|c|c|}
\hline \multirow[b]{2}{*}{ Site-Core-Section ${ }^{\mathrm{a}}$} & \multirow{2}{*}{$\begin{array}{l}\text { Depth }^{\mathrm{b}} \\
\text { (m) }\end{array}$} & \multicolumn{2}{|c|}{$\begin{array}{l}\text { Wet-Bulk Density } \\
\qquad(\mathrm{g} / \mathrm{cc})\end{array}$} & \multicolumn{2}{|c|}{$\begin{array}{l}\text { Porosity } \\
\text { (\% vol.) }\end{array}$} & \multirow{2}{*}{$\begin{array}{c}\text { Water } \\
\text { Content } \\
\text { (\% wt.) } \\
\text { Sample }^{\mathrm{d}}\end{array}$} \\
\hline & & $G a p e^{c}$ & Sample ${ }^{\mathrm{d}}$ & $G_{r a p e}{ }^{c}$ & Sample ${ }^{\mathrm{d}}$ & \\
\hline Average & 571 & 1.78 & - & 57 & - & - \\
\hline $105-35-1$ & 577 & 1.79 & 1.72 & 57 & 38 & 22 \\
\hline $105-35-2$ & 578 & 1.90 & - & 50 & - & - \\
\hline $105-35-3$ & 580 & 1.77 & - & 58 & - & - \\
\hline $105-35-4$ & 581 & 1.79 & - & 56 & - & - \\
\hline Average & 579 & 1.81 & 1.72 & 55 & 38 & 22 \\
\hline $105-36-1$ & 586 & 1.73 & - & 60 & - & - \\
\hline $105-36-2$ & 587 & 1.68 & - & 63 & - & - \\
\hline $105-36-3$ & 589 & 1.86 & - & 53 & - & - \\
\hline Average & 587 & 1.76 & - & 59 & - & - \\
\hline $105-37-1$ & 595 & 1.79 & - & 57 & - & - \\
\hline $105-37-2$ & 596 & 1.75 & - & 59 & - & - \\
\hline $105-37-3$ & 598 & 1.84 & - & 54 & - & - \\
\hline $105-37-4$ & 599 & 1.75 & - & 59 & - & - \\
\hline $105-37-5$ & 601 & 1.77 & - & 58 & - & - \\
\hline $105-37-6$ & 602 & 1.79 & - & 57 & - & - \\
\hline Average & 598 & 1.78 & - & 57 & - & - \\
\hline $105-38-1$ & 604 & 1.57 & - & 69 & - & - \\
\hline $105-38-2$ & 605 & 1.77 & - & 58 & - & - \\
\hline $105-38-3$ & 607 & 1.83 & - & 55 & - & - \\
\hline $105-38-4$ & 608 & 1.81 & - & 56 & - & - \\
\hline $105-38-5$ & 610 & 1.95 & - & 48 & - & - \\
\hline $105-38-6$ & 611 & 1.83 & - & 54 & - & - \\
\hline Average & 607 & 1.79 & - & 56 & - & - \\
\hline $105-39-1$ & 613 & 1.77 & - & 57 & - & - \\
\hline $105-39-2$ & 614 & 1.58 & - & 69 & - & - \\
\hline Average & 613 & 1.67 & - & 63 & - & - \\
\hline $105-41-1$ & 625 & 1.94 & - & 48 & & \\
\hline $105-41-2$ & 626 & 2.27 & - & 29 & & \\
\hline $105-41-3$ & 627 & 2.19 & - & 33 & & \\
\hline Average & 626 & 2.13 & - & 36 & & \\
\hline $105-42-1$ & 628 & 2.02 & - & 43 & & \\
\hline $105-42-2$ & 629 & 2.20 & - & 33 & & \\
\hline Average & 628 & 2.11 & - & 38 & & \\
\hline $105-43-1$ & 631 & 1.94 & - & 48 & & \\
\hline $105-43-2$ & 632 & 2.02 & - & 43 & & \\
\hline Average & 631 & 1.90 & - & 45 & & \\
\hline 106-1-1 & 1 & 1.29 & - & 85 & - & - \\
\hline $106-1-2$ & 2 & 1.42 & 1.44 & 78 & 68 & 47 \\
\hline $106-1-3$ & 3 & - & 1.50 & - & 68 & 45 \\
\hline $106-1-4$ & 5 & 1.42 & 1.51 & 77 & 68 & 45 \\
\hline $106-1-5$ & 7 & 1.55 & 1.64 & 70 & 67 & 41 \\
\hline Average & 4 & 1.42 & 1.52 & 77 & 68 & 45 \\
\hline $106-2-1$ & 46 & - & 1.68 & - & 59 & 35 \\
\hline $106-3-1$ & 111 & 1.56 & - & 70 & - & - \\
\hline $106-3-2$ & 112 & 1.55 & 1.70 & 71 & 56 & 33 \\
\hline $106-3-3$ & 114 & 1.61 & 1.76 & 67 & 52 & 30 \\
\hline
\end{tabular}


TABLE 4 - Continued

\begin{tabular}{|c|c|c|c|c|c|c|}
\hline \multirow[b]{2}{*}{ Site-Core-Section ${ }^{\mathrm{a}}$} & \multirow{2}{*}{$\begin{array}{c}\text { Depthb } \\
\text { (m) }\end{array}$} & \multicolumn{2}{|c|}{$\begin{array}{l}\text { Wet-Bulk Density } \\
(\mathrm{g} / \mathrm{cc})\end{array}$} & \multicolumn{2}{|c|}{$\begin{array}{l}\text { Porosity } \\
\text { (\% vol.) }\end{array}$} & \multirow{2}{*}{$\begin{array}{c}\text { Water } \\
\text { Content } \\
\text { (\% wt.) } \\
\text { Sample }\end{array}$} \\
\hline & & $\mathrm{Grape}^{\mathrm{c}}$ & Sample ${ }^{d}$ & Grape $^{c}$ & Sampled & \\
\hline $106-3-4$ & 115 & 1.71 & 1.78 & 62 & 49 & 28 \\
\hline $106-3-5$ & 117 & 1.80 & 1.78 & 56 & 40 & 23 \\
\hline $106-3-6$ & 118 & 1.74 & 1.87 & 60 & 39 & 21 \\
\hline Average & 114.5 & 1.66 & 1.78 & 64 & 47 & 27 \\
\hline 106-5-1 & 264 & 1.33 & 1.68 & 82 & 60 & 36 \\
\hline $106-5-2$ & 265 & 1.49 & 1.74 & 72 & 47 & 27 \\
\hline $106-5-3$ & 267 & 1.45 & 1.62 & 75 & 51 & 32 \\
\hline Average & 265 & 1.42 & 1.68 & 76 & 53 & 31 \\
\hline 106-6-1 & 340 & --- & 1.61 & --- & 50 & 31 \\
\hline 106B-1-1 & 367 & 1.54 & --- & 72 & --- & --- \\
\hline $106 \mathrm{~B}-1-2$ & 368 & 1.57 & --- & 70 & --- & --. \\
\hline $106 \mathrm{~B}-1-3$ & 370 & 1.61 & -.- & 68 & --. & -.- \\
\hline Average & 368 & 1.57 & ... & 70 & --- & -.- \\
\hline 106B-2-1 & 452 & 1.58 & --. & 71 & --- & $\cdots$ \\
\hline $106 \mathrm{~B}-2-2$ & 453 & 1.65 & -..- & 68 & --- & -.- \\
\hline $106 \mathrm{~B}-2-3$ & 455 & 1.75 & --. & 62 & -.- & --- \\
\hline 106B-2-4 & 456 & 1.79 & --- & 60 & --- & --- \\
\hline Average & 454 & 1.69 & --- & 65 & --- & $\cdots$ \\
\hline 106B-3-1 & 554 & 1.72 & -.- & 64 & -- & -.- \\
\hline $106 \mathrm{~B}-3-2$ & 555 & 1.73 & -.- & 64 & --- & --. \\
\hline 106B-3-3 & 557 & 1.76 & $\ldots$ & 62 & --- & $\cdots$ \\
\hline $106 \mathrm{~B}-3-4$ & 558 & 1.78 & --- & 61 & -.- & --- \\
\hline $106 \mathrm{~B}-3-5$ & 560 & 1.75 & --- & 62 & --- & -.- \\
\hline 106B-3-6 & 561 & 1.78 & --. & 61 & --- & -.- \\
\hline Average & 557 & 1.75 & --- & 62 & --- & $\ldots$ \\
\hline 106B-4-1 & 755 & 1.52 & ..- & 71 & -.- & --- \\
\hline $106 \mathrm{~B}-4-2$ & 756 & 1.73 &.-- & 58 & --- & --- \\
\hline $106 \mathrm{~B}-4-3$ & 758 & 1.77 & -.- & 55 & -- & --- \\
\hline 106B-4-4 & 759 & 1.85 & --. & 51 & --- & --- \\
\hline Average & 757 & 1.72 & -.- & 59 & --- & --- \\
\hline 106B-5-1 & 936 & 1.71 & -.- & 60 & --- & -.- \\
\hline $106 \mathrm{~B}-5-2$ & 937 & 1.68 & --. & 61 & --- & $\cdots$ \\
\hline $106 \mathrm{~B}-5-3$ & 939 & 1.62 & --- & 65 & --- & -.- \\
\hline $106 \mathrm{~B}-5-4$ & 940 & 1.65 & --- & 63 & --- & $\cdots$ \\
\hline $106 \mathrm{~B}-5-5$ & 942 & 1.64 & --- & 64 & --- & -.- \\
\hline 106B-5-6 & 943 & 1.72 & --- & 59 & --- & --- \\
\hline Average & 939 & 1.67 & --- & 62 & --- & $--\cdot$ \\
\hline 106B-6-1 & 955 & 1.62 & --- & 67 & --- & $\cdots$ \\
\hline 106B-6-2 & 956 & 1.75 & --- & 60 & --- & --- \\
\hline 106B-6-3 & 958 & 1.70 & --- & 63 & --- & --- \\
\hline Average & 956 & 1.69 & --- & 63 & --- & --- \\
\hline
\end{tabular}

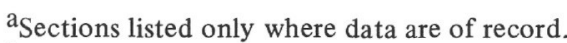

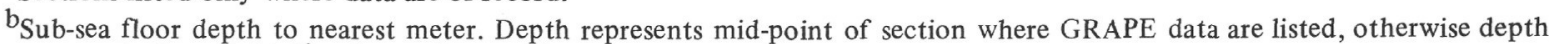
represents that of shipboard porosity sample.

${ }^{c}$ Average value per section from GRAPE computer data sheet.

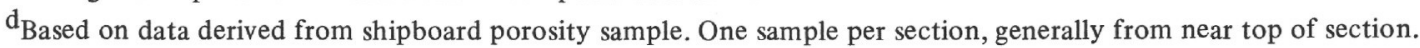

Porosity and water content calculations do not include salt correction. Some GRAPE porosities include a small increment caused by the use of $2.65 \mathrm{~g} / \mathrm{cc}$ for biosilica in the grain density computer program instead of $2.2 \mathrm{~g} / \mathrm{cc}$. This applies only to the following cores:

$$
\begin{array}{rlll}
98: & 1,5,6,8,9 & 104: & 3,4,6-10 \\
102: & 1-7,11 & 106: & 1,5,6, \text { B5, B6 } \\
103: & 4,6,7 & &
\end{array}
$$

The increment of porosity per cent ranges from an average of 1.4 at Site 103 to 5.4 at Site 104 (See footnotes, Table 6). See GRAIN DENSITY Section, or POROSITY AND WATER CONTENT Section, for discussion of influence on grain density on porosity calculations. 
natural gas could become mixed with the core material during the coring process. Air spaces develop when the core diameter is less than that of the core liner such as generally occurs during the coring of consolidated sediments. The annular space becomes filled with air during the on-deck core recovery operation. The principal effect of an error from these sources would be to lessen the wet-bulk density to something less than its actual value which in turn would make the porosity value higher than it actually should be for the sediment being measured. The effects from all three sources of error (drilling water, gas, air) are reflected in the GRAPE analog in column 5 and its derivative, the porosity plot, in column 4 .

Examples of the introduction of excessive amounts of drilling water into the core material can be seen in columns 4 and 5 of the physical property pages for the following cores from Site 105.

Core 7: The upper 7 meters contain what appear to be hole cavings consisting of slices, slabs, and chunks of clay tightly packed but containing an obviously large amount of interparticle space filled with drilling water. The bottom section of the core contained a sampling of in situ sediment consisting of a generally homogeneous clay. The porosity and the wet-bulk density plots for the bottom section appear normal.

Core 8: The bottom 2 meters of this core consist of hole cavings with water-filled interparticle spaces causing a reduction in the wet-bulk density plot and a consequent increase for the values in the porosity plot.

Core 13: All six sections contain hole cavings with water-filled interparticle spaces causing an irregular but overall reduction in the wet-bulk density plot.

Core 19: Although consisting of hole cavings, the clay in this core must have been very tightly packed. The wet-bulk density plot is less irregular but still somewhat reduced by the presence of admixed drilling water.

Core 31: The plots for the upper 2 meters are considerably reduced by the presence of a large amount of drilling water. This interval of core recovery consisted primarily of soupy drill cuttings.

Cores containing significant amounts of gas were recovered at Sites 102, 103, and 104. A large number of gas pockets developed in the core liners from these sites due to methane gas expansion. The gas pockets produced exceedingly low readings on the GRAPE analog and caused the porosity plot in column 4 of the physical property pages to show excessively high values. The cores which contained numerous voids due to gas expansion were generally not measured with the
GRAPE instrument. One such core was measured, however, and can be found on the physical property page for Core 13, Site 102.

An appreciable amount of air space is generally present between the surface of the core and the wall of the core liner in cases where core recovery consists of consolidated sediments such as limestone. Because the GRAPE device was calibrated to measure a full diameter core, the presence of the annular void space caused the wet-bulk density to appear significantly lower than what it should be for the sediment under investigation. Often, however, these hard rock cores were packed in disturbed core material derived from soft interbedded layers, so that core recovery consisted of alternations of filled and partially filled core liner. The sporadic packing of these cores is probably the main reason for the many extreme excursions on the density and porosity plots for the limestones and claystones from Site 105. The extreme excursions of the curves from Cores 21 and 22 (Site 105) are typical of the GRAPE records of less-than-standard diameter cores. Core 33 from Site 105 shows the effect that a lessening of annular void space has on the GRAPE device as it leaves the relatively small diameter hard limestone core in Section 1 and traverses the relatively softer limestone core of Sections 2 through 6. The coring process resulted in a more complete packing of the core liner throughout the sections containing the relatively softer material.

The physical property pages of the Site Reports (this volume) contain other examples of the effects of the admixed drilling water and natural gas and of the annular void space in partially filled core liners containing consolidated sediment.

Because of the numerous opportunities for the introduction of errors into the GRAPE derived porosity plot in column 4 of the physical property pages, it is recommended that this plot be used only to demonstrate general trends and ranges of porosity. Almost all of the porosity and water content measurements were made on disturbed sediments, thus they may not represent precise data of in situ conditions, but rather, overall approximate conditions. Maximum porosities and water contents are always suspected of being derived from highly disturbed sediments.

\section{Porosity Deviations: GRAPE Analog Versus Shipboard Porosity Sample}

Because the GRAPE porosity program used grain density values which were consistently higher than those calculated from the chemist's data derived from the individual shipboard porosity sample, and because the common sources of error in GRAPE measurements tend to increase porosity values, the resultant GRAPE porosities should be consistently higher than those 
derived from the shipboard samples. A comparison of the porosity values from these two sources was made by averaging the differences between 158 pairs of porosity values listed in the "GRAPE" and "Sample" columns of Table 4. A summary of the averaging process is found in Table 5. Of the 158 comparisons made only 11 showed GRAPE porosity to be less than the shipboard sample value. Eight of the eleven were from Site 105 and were generally only 2 or 3 porosity-per cent less than the shipboard sample value. Statistical analysis showed that 90 per cent of the Leg 11 GRAPE porosity values ranged from 2 porosity-per cent less than the shipboard sample values to 20 porosity-per cent greater than shipboard sample values for an average difference of 9 porosity-per cent by which GRAPE values exceeded shipboard sample values.

Figure 11 shows both sets of porosities plotted against depth for Site 106. According to the average porosity curves in Figure 11 the GRAPE porosities exceed the shipboard sample porosities by an amount varying from about 10 porosity-per cent for sediments above 100 meters to about 15 porosity-per cent for the deeper sediments. Table 5 shows a mathematical average difference of 15 porosity-per cent for Site 106. Core 1 at Site 106 had a mathematical average difference of 7 porosity-per cent. The curves shown in
Figure 11 were drawn independent of the mathematical averages, that is, their positions were based entirely on what appeared to be a "best fit".

A comparison of 158 pairs of wet-bulk density values from the GRAPE measurements and the shipboard samples listed in Table 4 showed by statistical analysis that 90 per cent of the Leg 11 GRAPE values ranged from $0.17 \mathrm{~g} / \mathrm{cc}$ less than shipboard sample values to $0.19 \mathrm{~g} / \mathrm{cc}$ greater than shipboard sample values for an average difference of $0.01 \mathrm{~g} / \mathrm{cc}$ by which GRAPE values exceeded shipboard sample values. A summary of the comparison is presented in Table 8 .

Because GRAPE wet-bulk density values were, on the average, quite close to the shipboard sample value, the principal reason for the GRAPE porosity values consistently exceeding the shipboard porosity values is probably the fact that the grain densities used in the GRAPE porosity computer program were consistently higher than the grain densities derived from the shipboard samples (See Table 6). Two contributing factors are thought to be responsible. First, a density of $2.85 \mathrm{~g} / \mathrm{cc}$ was used as an average value for completely dehydrated clay minerals. Subsequent to the calculations of grain densities and GRAPE porosities, information suggested that the average of $2.85 \mathrm{~g} / \mathrm{cc}$ may be a little high for clays on a world wide basis. An average

TABLE 5

Deviation Between GRAPE Porosity Values and Porosities Derived From Shipboard Samples

\begin{tabular}{|c|c|c|c|c|}
\hline \multirow[b]{2}{*}{ Hole } & \multirow{2}{*}{$\begin{array}{c}\text { Number of Sections } \\
\text { Studied }^{\mathrm{a}}\end{array}$} & \multicolumn{2}{|c|}{$\begin{array}{l}\text { Range of Deviation Per Section } \\
\text { (Porosity Per Cent) }\end{array}$} & \multirow{2}{*}{$\begin{array}{c}\text { Average Deviation } \\
\text { Per Site } \\
\text { (Porosity Per Cent) }\end{array}$} \\
\hline & & Upper Limit $b$ & Lower Limit $\mathrm{c}$ & \\
\hline 98 & 48 & 38.0 & 9.0 & $8.02(+)$ \\
\hline $99 \mathrm{~A}$ & 10 & 12.0 & 0.0 & $6.30(+)$ \\
\hline 100 & 5 & 6.0 & 0.0 & $3.40(+)$ \\
\hline $101 / 101 \mathrm{~A}$ & 23 & 19.0 & 0.0 & $12.40(+)$ \\
\hline 102 & 10 & 29.0 & 0.0 & $14.00(+)$ \\
\hline 103 & 12 & 25.0 & 0.0 & $10.25(+)$ \\
\hline 104 & 6 & 10.0 & 5.0 & $4.83(+)$ \\
\hline 105 & 33 & 26.0 & 7.0 & $5.79(+)$ \\
\hline 106 & 11 & 25.0 & 0.0 & $15.73(+)$ \\
\hline Average & & & & $8.75(+)$ \\
\hline
\end{tabular}

${ }^{\mathrm{a} B a s e d}$ on one set of porosity values per section of core. A set consists of the average GRAPE value per section and a value derived from one shipboard sample per section.

b Maximum amount by which GRAPE values exceed values derived from shipboard samples.

${ }^{c}$ Maximum amount by which GRAPE values were less than values derived from shipboard samples.

$\mathrm{d}_{(+)}=$GRAPE site average exceeded site average for shipboard samples.

It should be understood that the values listed as "upper" and "lower" limits represent extremes; for example, only five of 48 GRAPE porosities at Site 98 deviated from the shipboard sample values by more than 17 porosity per cent and only two of these five exceeded 19 porosity per cent. At Site 103, only two GRAPE porosities exceeded the shipboard sample value by more than 13 porosity per cent. Statistical analysis showed that 90 per cent of the Leg 11 GRAPE values ranged from 2 porosity-per cent less the shipboard sample values to 20 porosity-per cent greater than shipboard sample values for an average difference of 9 porosity-per cent by which GRAPE values exceeded shipboard sample values. *Reasons for the observed deviations are explained in the text, and are due largely to the mineral densities chosen for use in computation. 
TABLE 6

Average Grain Density Per Core for Leg 11 Sediments:

Results of Grain-Density Program Compared with Shipboard Sample Data

\begin{tabular}{|c|c|c|c|c|c|c|c|c|c|c|c|c|c|c|c|c|}
\hline \multirow[b]{2}{*}{ Hole } & \multicolumn{4}{|c|}{ Grain Density } & \multicolumn{4}{|c|}{ Grain Density } & \multicolumn{4}{|c|}{ Grain Density } & \multicolumn{4}{|c|}{ Grain Density } \\
\hline & Core & Shore $^{\mathrm{a}}$ & Ship $^{b}$ & Hole & Core & Shore $^{a}$ & Ship ${ }^{b}$ & Hole & Core & Shore $^{a}$ & Shipb & Hole & Core & Shore $^{a}$ & Ship b & Revised $^{c}$ \\
\hline \multirow[t]{16}{*}{98} & 1 & 2.73 & 2.32 & & 10 & - & - & \multirow{25}{*}{102} & 7 & 2.83 & - & 103 & 1 & 2.76 & 2.12 & \\
\hline & 2 & 2.71 & 2.35 & & 11 & 2.71 & - & & 8 & 2.83 & - & & 2 & 2.80 & 2.33 & \\
\hline & 3 & 2.71 & 2.48 & & 12 & 2.79 & - & & 9 & 2.71 & - & & 3 & 2.82 & 2.18 & \\
\hline & 4 & 2.71 & 2.53 & & 13 & 2.71 & - & & 10 & 2.78 & - & & 4 & 2.79 & 2.29 & \\
\hline & 5 & 2.70 & 2.22 & & 14 & 2.74 & - & & & & & & 5 & 2.85 & 2.26 & \\
\hline & 6 & 2.71 & 2.52 & & & & & & 1 & 2.75 & 2.58 & & 6 & 2.80 & - & \\
\hline & 7 & 2.71 & 2.56 & 100 & 1 & 2.71 & 2.68 & & 2 & 2.71 & 2.36 & & 7 & 2.80 & - & \\
\hline & 8 & 2.69 & 2.40 & & 2 & 2.74 & - & & 3 & 2.77 & 2.41 & & & & & \\
\hline & 9 & 2.69 & 2.44 & & 3 & 2.75 & - & & 4 & 2.77 & 2.67 & 104 & 1 & 2.77 & 2.38 & \\
\hline & 10 & 2.64 & 2.54 & & 4 & 2.70 & - & & 5 & 2.73 & 2.28 & & 2 & 2.80 & 2.45 & \\
\hline & 11 & 2.49 & 2.35 & & 5 & 2.75 & - & & 6 & 2.72 & 2.15 & & 3 & 2.72 & 1.97 & 2.46 \\
\hline & 12 & 2.64 & 2.50 & & 6 & 2.75 & - & & 7 & 2.75 & 2.14 & & 4 & 2.74 & 2.28 & 2.49 \\
\hline & 13 & 2.68 & - & & 7 & 2.71 & - & & 8 & - & 2.36 & & 6 & 2.72 & 2.09 & 2.42 \\
\hline & 14 & 2.71 & - & & 8 & 2.71 & - & & 9 & 2.84 & 2.43 & & 7 & 2.72 & 2.25 & 2.43 \\
\hline & 15 & 2.71 & - & & 9 & 2.71 & - & & 10 & 2.84 & 2.23 & & 8 & 2.72 & 1.41 & 2.43 \\
\hline & & & & & 10 & 2.72 & - & & 11 & 2.85 & 1.99 & & 9 & 2.72 & 2.20 & 2.45 \\
\hline \multirow[t]{9}{*}{$99 \mathrm{~A}$} & 1 & 2.75 & 2.33 & & & & & & 12 & 2.85 & 2.44 & & 10 & 2.79 & 1.92 & 2.52 \\
\hline & 2 & 2.73 & 2.43 & 101 & 1 & 2.90 & 2.30 & & 13 & 2.85 & 2.36 & & & & & \\
\hline & 3 & - & - & & 2 & 2.97 & 2.36 & & 14 & 2.89 & 2.25 & 105 & 1 & - & - & \\
\hline & 4 & - & - & $101 \mathrm{~A}$ & 1 & 3.02 & 2.38 & & 15 & 2.89 & 1.96 & & 2 & $(2.80)$ & 2.62 & \\
\hline & 5 & - & - & & 2 & 3.06 & 2.42 & & 16 & 2.89 & 1.97 & & 3 & 2.80 & 2.48 & \\
\hline & 6 & - & - & & 3 & 2.93 & 2.42 & & 17 & 2.89 & 2.31 & & 4 & 2.81 & 2.19 & \\
\hline & 7 & - & - & & 4 & 2.83 & 2.46 & & 18 & 2.92 & 2.23 & & 5 & 2.45 & 2.61 & \\
\hline & 8 & 2.71 & 2.42 & & 5 & 2.83 & 2.23 & & 19 & 2.89 & 2.20 & & 6 & $(2.83)$ & 2.29 & \\
\hline & 9 & 2.71 & 2.55 & & 6 & 2.83 & - & & & & & & 7 & $(2.83)$ & 2.47 & \\
\hline
\end{tabular}


TABLE 6 - Continued

\begin{tabular}{|c|c|c|c|c|c|c|c|c|c|c|c|c|c|c|}
\hline \multirow[b]{2}{*}{ Hole } & \multicolumn{4}{|c|}{ Grain Density } & \multicolumn{5}{|c|}{ Grain Density } & \multicolumn{5}{|c|}{ Grain Density } \\
\hline & Core & Shore $^{\mathrm{a}}$ & Ship $b$ & Revised $^{c}$ & Hole & Core & Shore $^{a}$ & Ship $^{a}$ & Revised $^{c}$ & Hole & Core & Shore $^{a}$ & Ship $^{b}$ & Revised $^{\mathrm{C}}$ \\
\hline & 8 & 2.78 & 2.57 & & & 25 & 2.79 & - & & & 42 & - & - & \\
\hline & 9 & 2.66 & 2.41 & & & 26 & $(2.77)$ & - & & & 43 & - & - & \\
\hline & 10 & 2.78 & 2.40 & & & 27 & 2.74 & - & & 106 & 1 & 2.77 & 2.61 & 2.72 \\
\hline & 11 & 2.51 & - & & & 28 & 2.71 & - & & & 2 & 2.75 & 2.65 & \\
\hline & 12 & 2.53 & 2.42 & & & 29 & 2.71 & - & & & 3 & 2.76 & 2.49 & \\
\hline & 13 & omit & 2.35 & & & 30 & 2.71 & - & & & 4 & 2.80 & - & 2.71 \\
\hline & 14 & 2.53 & - & & & 31 & 2.71 & - & & & 5 & 2.77 & 2.45 & 2.71 \\
\hline & 15 & 2.59 & 2.06 & & & 32 & 2.71 & - & & & 6 & 2.71 & 2.23 & 2.51 \\
\hline & 16 & (2.53) & - & & & 33 & 2.77 & - & & 106B & 1 & 2.78 & - & \\
\hline & 17 & 2.66 & - & & & 34 & 2.77 & - & & & 2 & 2.85 & - & \\
\hline & 18 & 2.71 & - & & & 35 & 2.79 & 2.17 & & & 3 & 2.85 & - & \\
\hline & $\begin{array}{l}19 \\
20\end{array}$ & $\begin{array}{l}\text { omit } \\
2.75\end{array}$ & $\begin{array}{c}2.53 \\
-\end{array}$ & & & $\begin{array}{l}36 \\
37\end{array}$ & $\begin{array}{l}2.78 \\
2.79\end{array}$ & $\begin{array}{l}- \\
-\end{array}$ & & & 4 & 2.94 & - & \\
\hline & 22 & $(2.75)$ & - & & & 39 & 2.79 & - & & & 6 & 2.72 & - & 2.42 \\
\hline & 23 & 2.72 & - & & & 40 & - & - & & & 7 & 2.85 & - & \\
\hline & 24 & 2.71 & - & & & 41 & - & - & & & 8 & 2.85 & - & \\
\hline
\end{tabular}

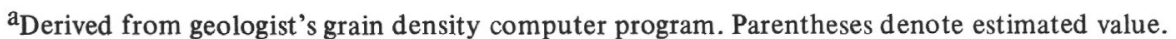

${ }^{b}$ Derived from shipboard porosity sample (no salt correction).

${ }^{c_{R}}$ Recalculation of grain density for biosilica-rich cores. This revision, added in proof, was based on $2.2 \mathrm{~g} / \mathrm{cc}$ for biosilica in the grain density computer program instead of $2.65 \mathrm{~g} / \mathrm{cc}$. The average decrease in grain densities for the revised core averages per Site is listed below. Approximate equivalent porosity is based on nomograms published by Harms and Choquette (1965).

$\begin{array}{rr}\text { Site } & \rho 8 \\ 98 & 0 . \\ 102 & 0.14 \\ 103 & 0.07 \\ 104 & 0.27 \\ 106 & 0 .\end{array}$

$\begin{array}{cl}\rho \mathrm{g} & \phi \\ 0.12 & 2.4 \\ 0.14 & 2.8 \\ 0.07 & 1.4 \\ 0.27 & 5.4 \\ 0.12 & 2.5 \text { (includes 106B) }\end{array}$

d The following core analysis data were provided through the courtesy of Core Laboratories, Inc., Dallas, Texas. The lower "Shore" density is probably due to the zeolite component introduced into the grain density program whereas the Graphic Chart for Site 98 (Figure 4, Site Report, this volume) shows that the zeolite component is most likely absent in the interval from which the Core Lab samples were taken.
Sample
$98-13-1 ; 139-140$

$\begin{array}{cc}\phi & \mathrm{P} \\ 47.3 & 6.2 \\ 35.4 & 250.0\end{array}$
$\rho \mathrm{g}$
2.72
2.72

$98-12-1 ; 20-22$ 
value of $2.80 \mathrm{~g} / \mathrm{cc}$ might have been more generally representative of completely dehydrated clay minerals. It should be recognized, however, that the densities of completely dehydrated clay minerals vary from 2.75 $\mathrm{g} / \mathrm{cc}$ to $3.10 \mathrm{~g} / \mathrm{cc}$ (Poupon et al., 1970). Using the value of $2.80 \mathrm{~g} / \mathrm{cc}$ would have reduced the GRAPE porosities by not more than about 3 porosity per cent which means that, on the average, Leg 11 GRAPE porosities would still have been higher than porosities derived from shipboard samples by about 6 porosity per cent. The second contributing factor is the fact the quartz value of 2.65 was used as the mineral density for biogenous silica. It was subsequently realized that the opal value of 2.2 would be more applicable. Thus the obscured discrepancies between calculated GRAPE porosities and porosities measured on board ship are thought to be mostly a function of the clay and silica mineral densities chosen.

The grain densities for the biosilica-rich cores were recalculated and added in proof to TABLE 6. (See "Revised" column.) The revised grain densities are lower than those derived from original calculations by an over all average increment of $0.14 \mathrm{~g} / \mathrm{cc}$. This increment would, according to nomograms published by Harms and Choquette (1965), amount to about 3 porosity per cent.

\section{Porosity-Depth Crossplots}

Porosity-depth crossplots were made for the carbonate sediments at Site 98 (Figure 8) and the silty clays at Sites 102, 105 and 106 (Figures 9, 10 and 11). The average porosity curves for these plots are used in this chapter to illustrate and to measure the effects of: (1) compaction, a primary factor in the consolidation of clays, and (2) carbonate diagenesis, a dominant factor in the consolidation of carbonate sediments. The reader is referred to the Sediment Consolidation section of this chapter for a discussion of the interrelationships of Leg 11 porosity and velocity measurements and their significance with regards to sediment consolidation.

According to the average porosity curves based on shipboard sample data for the clays at Sites 102 and 106 (Figures 9 and 11), near-sea floor porosities averaged about 78 and 68 per cent, respectively, for the first meter of sediments. These high porosities decreased rapidly with depth until at about 120-130 meters porosity averaged about 52 per cent. Below the 130 -meter level porosity decreased with depth at a much slower rate. The average clay-porosity curve for Site 105 (Figure 10) does not seem to reflect the change in rate of porosity decrease at the 130-meter level. Possible reasons for this are discussed in the Sediment Consolidation section of this chapter.
Porosity values associated with the marked change in the slope of the average porosity curves at Sites 102 and 106 are near the lower limit of Weller's porosity range of 80 to 45 per cent. According to Weller (1959), the water films which completely surround the clay mineral grains in the high porosity sediments have been squeezed aside in the lower porosity sediments so that the clay mineral grains make contact with each other. This grain contact would add a new element of strength making it possible for the sediment to offer a marked increase in resistance to further compaction. The steeper slope of the average porosity curves below 130 meters is interpreted as a reflection of the increased resistance to compaction due to grain contact. Further discussion of Weller's compaction studies and their relation to Leg 11 data are found in the Sediment Consolidation section of this chapter.

\section{GRAIN DENSITY COMPUTER PROGRAM FOR LEG 11 SEDIMENTS}

An average grain density was calculated by the writer for each of the cores listed in Table 6 . The densities were derived from computations based on: (1) shipboard estimates of per cent mineral composition (See Graphic Charts, Figure 4 in Site Reports, this volume), (2) shore based laboratory carbonate analyses, and (3) mineral densities (Table 7). The writer thought it preferable to develop grain densities which were based on the petrology of the cores under investigation rather than use previously determined values for sediments from depositional basins other than those in which Leg 11 sites were located.

Variations in rock type from one sediment source area to another might produce significant variations in the mineral composition of the sediments. Several cores from Sites 105 and 106, for example, appear to contain significant amounts ( 5 per cent \pm ) of rutile or other heavy minerals. Pelagic deposition could also vary significantly from one area to another with the result that hemipelagic muds in one area might be rich in biosilica (radiolarians, sponge spicules, diatoms), whereas, the muds of another area might contain an abundance of calcareous organisms and little or none of the siliceous fauna; for example, coring at Sites 102 , 103 and 104 encountered heavy concentrations of siliceous fauna in certain zones. Similar geographic variations in sediment source areas and pelagic deposition were noted by Hamilton (1970). He concluded that grain matrix densities for sediments in one depositional basin should not be used in computations involving sediments from a different basin.

In order to establish grain densities based on Leg 11 core data, a computer program was developed by $\mathrm{E}$. R. Blakeman (The Superior Oil Company) to handle the data from the Graphic Charts, the shore-based 


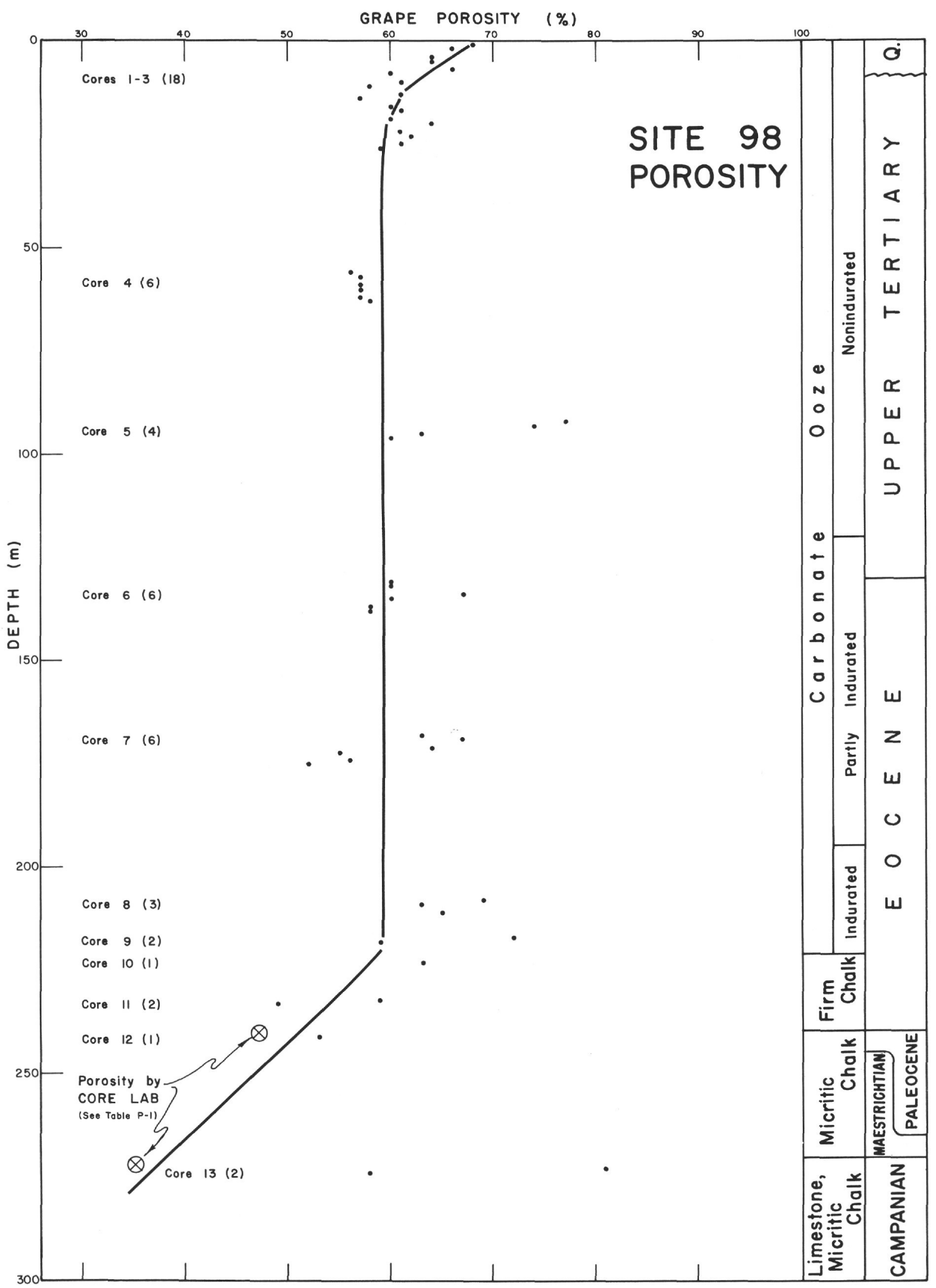

Figure 8. Porosity-depth crossplot for carbonate sediments at Site 98. Each point represents the average GRAPE porosity per section of core. Two plots, $\otimes$, represent core analyses by Core Laboratories, Inc. (Dallas, Texas). 


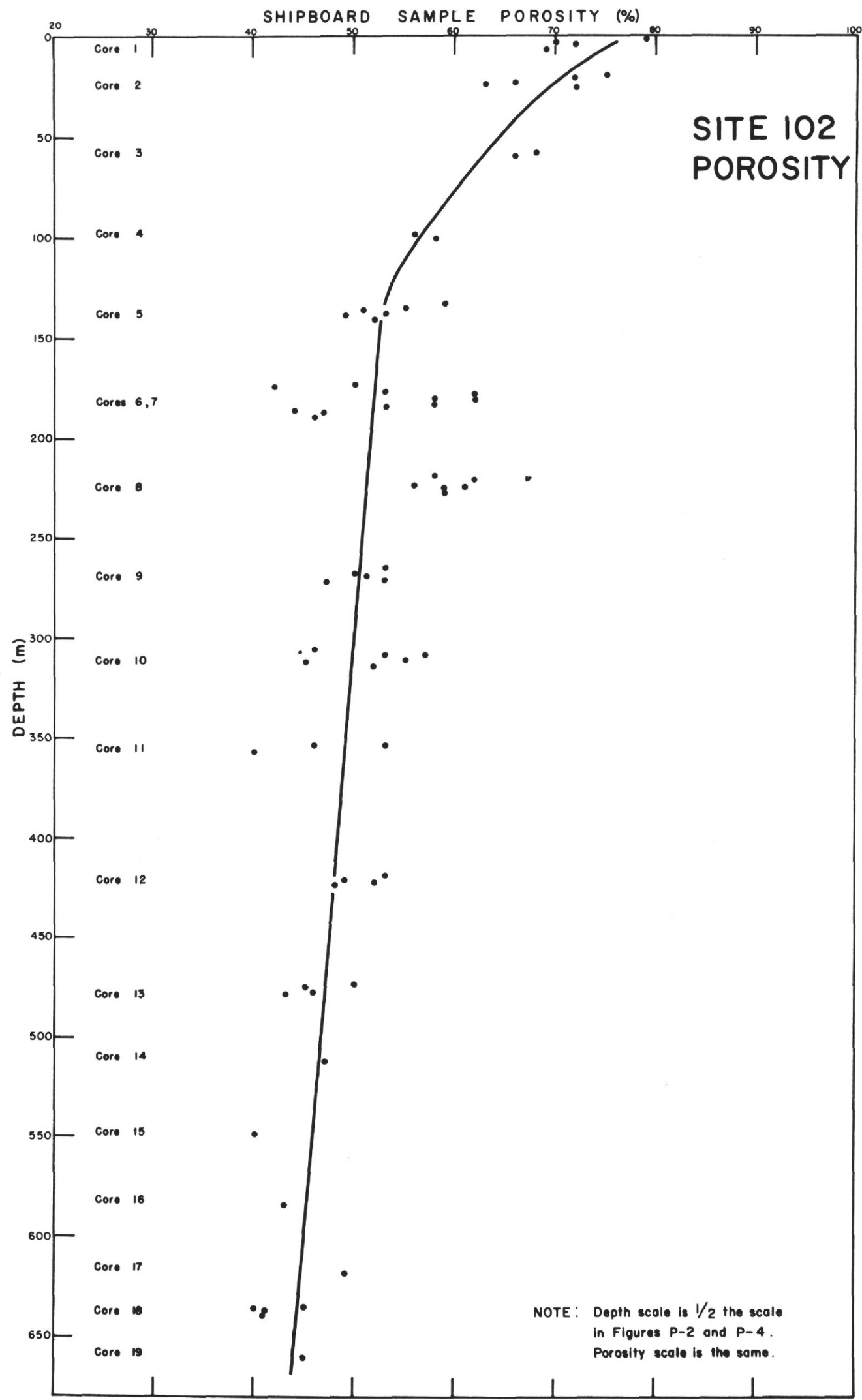

Figure 9. Porosity-depth crossplot for silty clays at Site 102. Each point represents a value derived from a single shipboard sample per section of core. The slope change in the average porosity curve at about $130 \mathrm{~m}$ is interpreted as reflecting a change in the rate of compaction in clays from a more rapid rate above to a slower rate below the 130-m depth. 


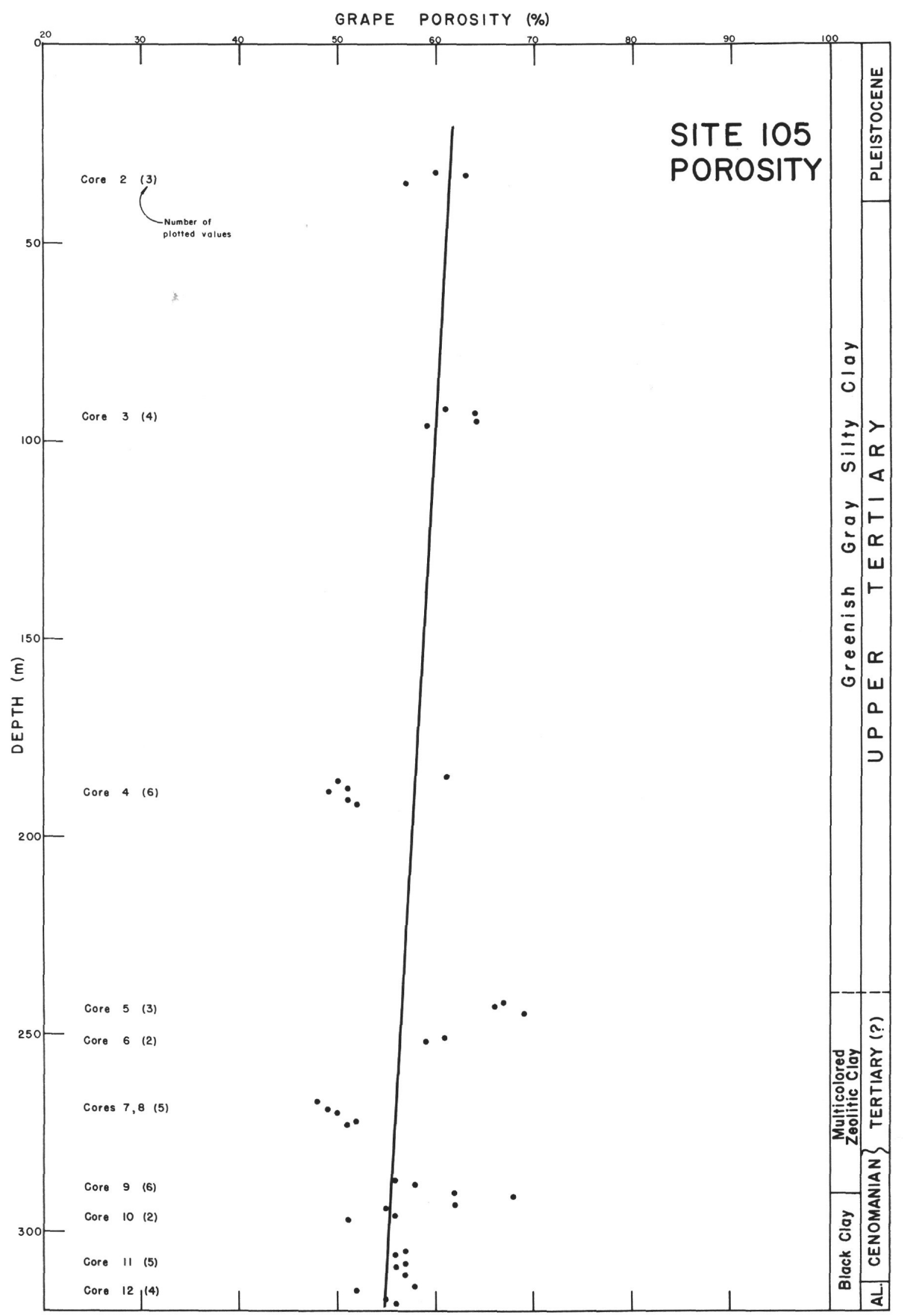

Figure 10. Porosity-depth crossplot for silty clays at Site 105. Each point represents the average GRAPE porosity per section of core. 


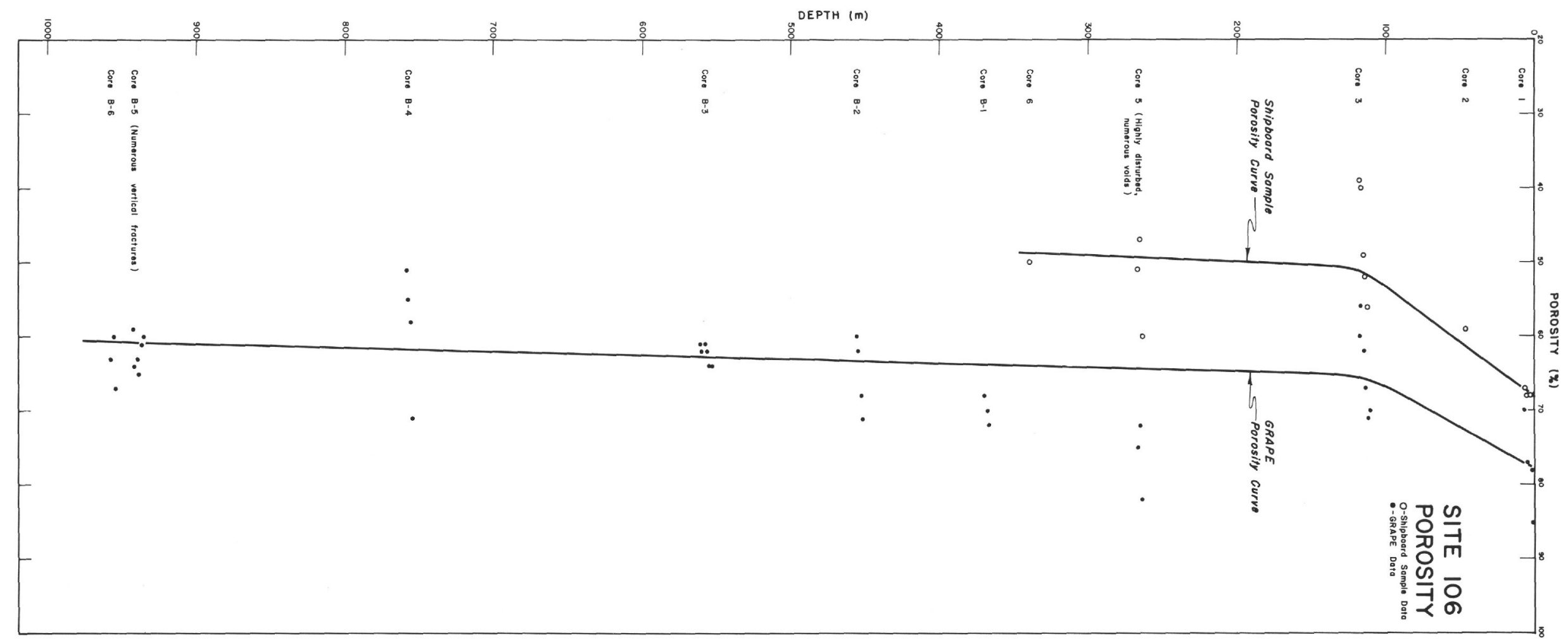

Figure 11. Porosity-depth crossplot for silty clays at Site 106. Each open circle represents a value derived from a single shipboard sample per section of core. Each solid dot represents the average GRAPE value per section of core. The average curves were drawn independent from the mathematical average difference listed in Table 5. The slope change in the average porosity curve at about $120 \mathrm{~m}$ is interpreted as reflecting a change in the rate of compaction in clays from a more rapid rate above to a slower rate below the 120-m depth. 
TABLE 7

Mineral Densities

\begin{tabular}{|c|c|c|c|}
\hline Minerals & Leg $11^{\mathrm{a}}$ & $\begin{array}{l}\text { Electron } \\
\text { Density }\end{array}$ & $\begin{array}{l}\text { Specific } \\
\text { Gravity } \\
\end{array}$ \\
\hline Calcite & 2.71 & 2.71 & 2.715-2.94 \\
\hline Dolomite & 2.82 & 2.82 & 2.86 \\
\hline Aragonite & 2.93 & 2.93 & $2.94-2.95$ \\
\hline Siderite & 3.91 & 3.91 & 3.96 \\
\hline Clay & 2.85 & & \\
\hline Quartz & 2.65 & 2.65 & 2.65 \\
\hline Muscovite & 2.79 & 2.79 & $2.77-2.88$ \\
\hline Glauconite & $2.7 \pm$ & & 2.4-2.95 \\
\hline Pyrite & 4.08 & 4.08 & 5.018 \\
\hline Zeolite group & 2.11 & & \\
\hline clinoptilolite & $(2.11)$ & & \\
\hline phillipsite & & & 2.2 \\
\hline heulandite & & & $2.1-2.2$ \\
\hline Geothite & 3.80 & & $3.3-4.3$ \\
\hline Hematite & 5.10 & & 5.26 \\
\hline $\begin{array}{l}\text { Feldspar group } \\
\text { microcline } \\
\text { oligoclase }\end{array}$ & & & $\begin{array}{l}2.56-2.63 \\
2.63-2.65\end{array}$ \\
\hline "Heavy" group & 3.90 & & \\
\hline rutile & $(4.20)$ & & $4.23-5.5$ \\
\hline sphalerite & $(4.00)$ & & $3.9-4.1$ \\
\hline amphiboles & $(3.20)$ & & \\
\hline hornblende & & & $3.02-3.45$ \\
\hline actinolite & & & $3.02-3.44$ \\
\hline glaucophane & & & $3.08-3.30$ \\
\hline \multicolumn{4}{|c|}{$\begin{array}{l}\text { a Values used in computation of grain densities for Leg } 11 \text { sediments. Values in parentheses were used to } \\
\text { determine representative values for the groups. The clay value is a general average for completely dehydrated } \\
\text { clay minerals (Poupon, } 1970 \text { ), and the quartz value was used for the biosilica fraction of siliceous sediments. } \\
\text { (The reader is referred to the text for a discussion of the applicability of these figures). The clinoptilolite } \\
\text { value is from Mason and Sand (1960). The goethite value was used for the limonite fraction. The feldspar } \\
\text { fraction of the sediments was minor and was included with the quartz fraction when present. }\end{array}$} \\
\hline \multicolumn{4}{|c|}{ b Harms and Choquette (Trans. 6th Ann. SPWLA Loggin Sym., Dallas, 1965). } \\
\hline
\end{tabular}

laboratory carbonate analyses, and the mineral densities from Table 7. Every effort was made to be as objective as possible in the development of this program.

Each of the mineralogical and faunalistic categories in the original shipboard Graphic Charts was subdivided into four parts according to the degree of abundance as determined by petrographic microscope examination of smear slide samples. The four subdivisions and their percentage limits consisted of: rare ( 0 to 5 per cent); common ( 5 to 15 per cent); abundant ( 15 to 75 percent); dominant ( 75 to 100 per cent). The Graphic Charts published in this volume are based on these subdivisions and percentage limits. Smear samples of lithologies with limited extent (for example, occasional small lenses of siderite) as well as the entire "rare" classification were eliminated from consideration in the grain density computer program. Only those smear samples considered representative of the section of core from which they were taken were used in the program.

As mentioned earlier, biosilica was in some instances an important component of the sediments in Leg 11 cores. The siliceous fossils constitute individual categories in the Graphic Charts. Each of these was treated as a mineral category with a mineral density equal to that of quartz. 
A mineral density of $2.2 \mathrm{~g} / \mathrm{cc}$ would have been more representative of the opaline characteristics of the biosilica. The grain densities for the biosilica-rich cores were recalculated and added in proof to Table 6 (See "Revised" column). The revised grain densities are lower than those derived from original calculations by an over all average increment of $0.14 \mathrm{~g} / \mathrm{cc}$. This increment would, according to nomograms published by Harms and Choquette (1965), amount to about 3 porosity per cent.

The calcite fraction of Leg 11 cores is represented in the Graphic Charts primarily in terms of the faunal content of the sediments. In order to determine what per cent of calcite should be introduced into the grain density computer program, it was first necessary to determine the per cent of carbonate in each smear slide sample. A geologist's estimate of the total carbonate in each smear sample was recorded on board and subsequently filed with other key-punch card data. The estimate included contributions from calcite, dolomite, siderite, and in the case of Site 98, some aragonite. The occurrence of dolomite, siderite, and aragonite were recorded separately in the original shipboard Graphic Charts. (Aragonite was removed from the published version of these charts since it was significant only at Site 98.) The computer program summed the percentages of dolomite, siderite, and aragonite, and then subtracted this sum from the total carbonate in order to determine the proportionate amount of the calcite component. When totaling the dolomite, siderite and aragonite fractions, only the common, abundant, and dominant categories were used. Percentage values were arbitrarily assigned to these categories as follows: common $=5$; abundant $=15$; dominant $=100$.

The entire calcite derivation operation was built into the grain density computer program along with a correction factor which would be applied to the geologist's estimate of total carbonate. The geologist's estimate was early suspected of being too high. A plot of the geologist's core average versus the shorebased laboratory core average confirmed this suspicion (Figure 12). Also included in the plot were the individual geologist's estimates and shore-based carbonate analyses where these two samplings were not more than 5 centimeters apart in the core. It should be mentioned here that the shore-based carbonate analyses, like the geologist's carbonate estimate, represent total carbonate $\left(\mathrm{CaCO}_{3}, \mathrm{MgCO}_{3}\right.$, $\mathrm{FeCO}_{3}$, etc.). A fourth order polynomial equation, derived from the "best fit" curve which was hand drawn on the carbonate plot (Figure 12) was added to the grain density computer program to make the geologist's carbonate estimate fit more closely the laboratory results. (This curve constitutes the correction factor mentioned above.) Use of the geologist's data was deemed desirable because of the higher sampling frequency, as compared to the lower frequency of the laboratory carbonate analyses.

One or more values of grain density were obtained for most sections of Leg 11 cores through the application of the grain density computer program. The section values of each core were averaged and used in the shore-based GRAPE porosity program which produced the plot shown in column 4 of the physical property pages in the Site Reports of this volume.

The grain density core averages derived from the grain density computer program and the core averages derived from the chemist's data which were obtained from a small sample generally from near the top of a section of core are listed in Table 6 in the "SHORE" and "SHIP" columns respectively. A comparison of the densities in these two columns showed that the chemist's densities were, on the average, about 16 per cent lower than the computer derived densities, with a range of about 2 to 32 per cent. The discrepancy is largely due to the mineral densities chosen for the clay mineral and biogenous silica (see discussion in the last two paragraphs of Section 4 of POROSITY AND WATER CONTENT). A recalculation of the average difference, incorporating the revised computer derived densities which were added in proof to Table 6 (See "Revised" column) showed that the chemist's densities were still lower by an over all average difference of about 14 per cent.

The grain density computer program was proven basically sound in that it did provide representative grain density values for use in the shore-based GRAPE porosity computer program. It was apparent, however, that the grain density program does need certain refinements. The program might also be benefited by certain adjustments in the shipboard acquisition and compilation of raw data.

\section{WET-BULK DENSITY}

The Gamma-Ray Attenuation Porosity Evaluator (GRAPE) was used on board ship to measure the wet-bulk density of the sediments in 85 of the 153 Leg 11 cores. Most of the unmeasured cores had little or no recovery. Wet-bulk density values were also calculated from the small individual shipboard porosity samples taken from 74 of the Leg 11 cores. All wet-bulk density values are listed in Table 4. Figure 7 graphically shows the distribution of wet-bulk density data for Leg 11 cores.

A comparison of 158 GRAPE wet-bulk density values with those derived from the individual shipboard samples showed GRAPE values averaging very close to the shipboard sample values. The overall average difference amounted to only $0.016 \mathrm{~g} / \mathrm{cc}$. 


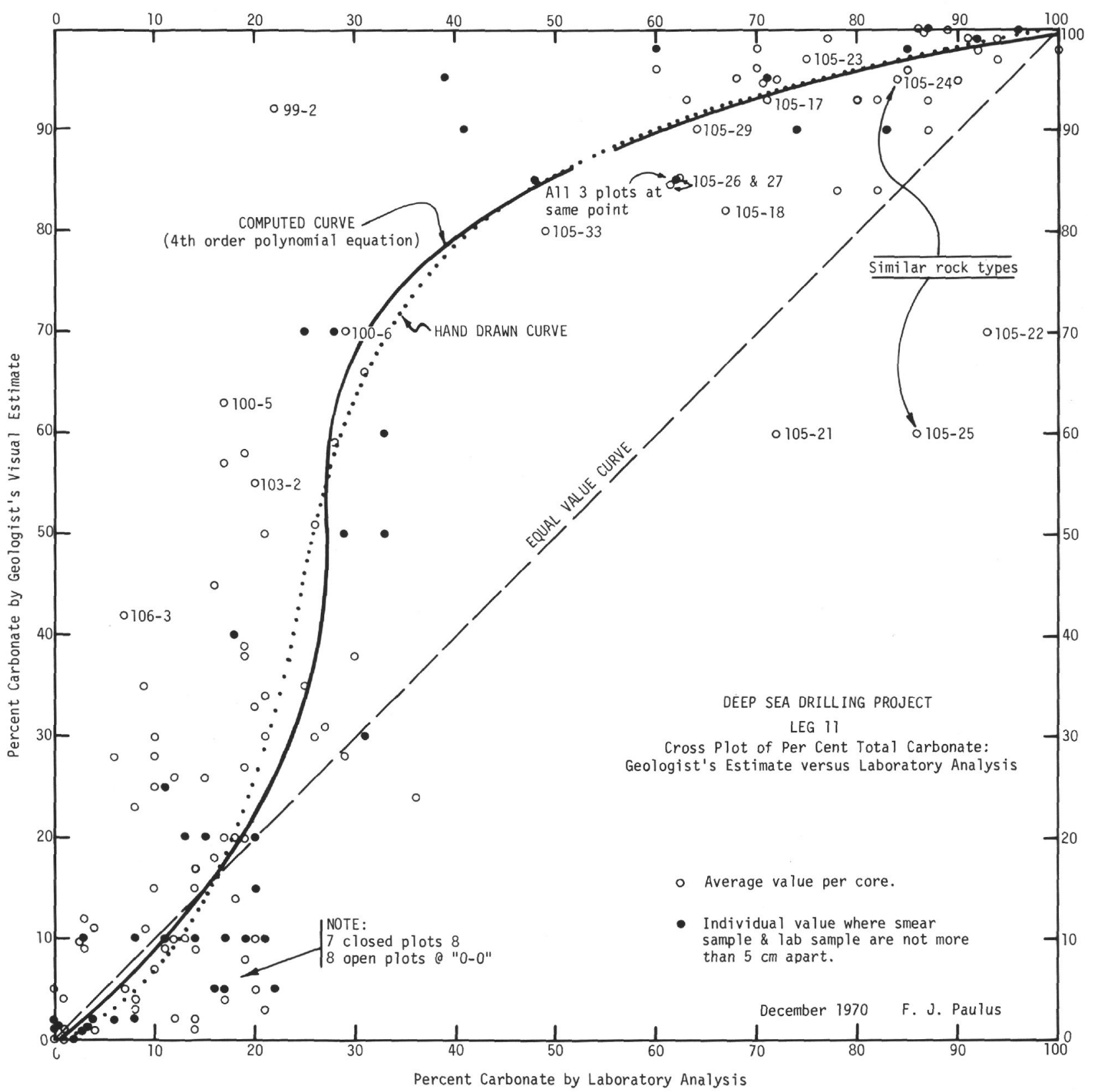

Figure 12. Cross plot of the total carbonate as estimated by the geologist during the petrographic examination of smear slide samples and the total carbonate as indicated by shore based laboratory carbonate analysis of Leg 11 core samples. The open circle represents a cross plot of the geologist's core average and the laboratory average for the corresponding core. The solid circle is a cross plot of the geologist's carbonate estimate for an individual smear slide sample and a single laboratory analysis from the corresponding core where that analysis was at or near (within $5 \mathrm{~cm}$ ) the depth of the smear sample. The cross plot was constructed in an attempt to determine to what degree the geologist's estimate deviated from the laboratory analysis. The average deviation from the Equal Value curve is represented by the Hand Drawn curve. The Computed curve was determined so as to be able to introduce the average deviation into the grain density computer program as a correction factor for the geologist's carbonate estimate. Some of the core average plots are identified as to Site and core number. 
The maximum amount by which GRAPE values were either greater than or less than the values derived from the shipboard samples are listed in Table 8 along with the average amount and direction of deviation for each Leg 11 site. Statistical analyses showed that 90 per cent of Leg 11 GRAPE values ranged from $0.17 \mathrm{~g} / \mathrm{cc}$ less than shipboard sample values to $0.19 \mathrm{~g} / \mathrm{cc}$ greater than shipboard sample values for an average difference of $0.01 \mathrm{~g} / \mathrm{cc}$ by which GRAPE values exceeded shipboard sample values.

Almost all of the wet-bulk density measurements were made on disturbed sediments; thus, they may not represent precise data of in situ conditions, but rather, overall approximate conditions. Minimum wet-bulk densities are always suspected of being derived from highly disturbed sediments. The errors which may be introduced into the GRAPE measurements result mainly from: (1) the mixing of drilling water and natural gas with the sediments during the coring operation, and (2) the air filled space between the surface of the core and the inner wall of the core liner. A discussion of these errors and their effects can be found in the Porosity and Water Content section of this chapter.

The GRAPE information was recorded in the form of a continous analog graph. A computer derived plot of this analog is presented in column 5 of the physical property pages of the Site Reports (this volume). The GRAPE method of measuring wetbulk density is based on the gamma-ray scattering caused by the sediment sample. The measurement corresponds to an evaluation of the number of electrons per unit volume. The design and use of the GRAPE equipment have been described by Evans (1965). Harms and Choquette (1965) have written a geologic evaluation of the device. The shipboard GRAPE unit has been described in DSDP Volume 2 (Peterson, M. N. A. et al., 1970).

The wet-bulk density parameter has been thoroughly studied because of its close relations to other physical properties, such as, porosity and velocity. Numerous references to these studies have been listed in the selected bibliography at the end of this chapter. Generally, wet-bulk density shows an inverse relationship to velocity. The inverse relationship is well illustrated by a comparison of columns 4 and 5 of the physical property pages in the Site Reports (this volume). Occasionally the consolidation history of a sedimentary section includes a sequence of events which will produce an anomalous relationship between certain physical property parameters. Such is apparently the case at Site 105 where the average wet-bulk density curve plots out as a vertical line throughout the silty clay section (Figure 13). The irregular density-depth relations in
Figure 13 are attributed, in part, to the influence of varying densities of different mineral associations, some of which are described in Figure 16 (see Sediment Consolidation section in this chapter).

\section{SOUND VELOCITY}

\section{Leg 11 Sound Velocity Program}

Sound velocity measurements were made on the core while it was still in the plastic liner. The velocity for each core section was measured at ambient laboratory temperature about four hours after core recovery. Velocity measurements were not corrected to in situ conditions nor to any other standard. The application of such corrections is discussed by Hamilton et al. (1956), Hamilton (1963, 1965, 1970, and 1971), Sutton et al. (1957), Laughton (1954, 1957), and Shumway (1958, 1960).

Velocity measurements were made on cores from eight Leg 11 drill sites. Only two sites (98, 105) have measurements extending from near the sea floor to below 200 meters. The depth range of the velocity measurements at each site is shown in Table 9. The distribution of velocity measurements throughout each hole is shown on the hole-scale physical property page for each site (Site Reports, this volume).

Virtually all unconsolidated and some semiconsolidated cores were disturbed during coring to the point of being contorted and tightly packed inside the plastic core liner. This profound reorientation of original sedimentary structures has undoubtedly had some effect on the velocity measurements. Therefore, even after temperature-pressure corrections, these measurements may still not represent exact in situ velocities. Although these measurements may not be precise and may vary widely within any given core, they still may be useful in the determination of an average velocity-depth curve for the sediments penetrated. The average velocity curve can be used in conjunction with the average porosity curve to determine the compaction correction trend for use in sediment consolidation studies. The average velocity curve might also be used for approximate time-depth conversions of seismic profile reflection time.

\section{Measurement of Velocity}

A pulse method was used to apply a $400 \mathrm{kHz}$ frequency at each of three positions (35.5, 75.0 and $112.5 \mathrm{~cm}$ ) along the 1.5 -meter core section. The average velocity for each of the three positions formed the basis for the velocity plot in column 6 of the physical property pages for the Site Reports in this volume. 
TABLE 8

Deviation GRAPE Wet-Bulk Density Values and Wet-Bulk Densities Derived From Shipboard Samples ${ }^{\mathrm{a}}$

\begin{tabular}{|c|c|c|c|c|}
\hline \multirow[b]{2}{*}{ Hole } & \multirow{2}{*}{$\begin{array}{l}\text { Number of Sections } \\
\text { Studied }\end{array}$} & \multicolumn{2}{|c|}{$\begin{array}{l}\text { Range of Deviation Per Section } \\
\qquad(\mathrm{g} / \mathrm{cc})\end{array}$} & \multirow{2}{*}{$\begin{array}{l}\text { Average Deviation } \\
\text { Per Site } \\
\text { (g/cc) }\end{array}$} \\
\hline & & Upper Limit $b$ & Lower Limit ${ }^{c}$ & \\
\hline 98 & 48 & 0.210 & 0.290 & $0.002(+)$ \\
\hline $99 \mathrm{~A}$ & 10 & 0.100 & 0.080 & $0.016(+)$ \\
\hline 100 & 5 & 0.080 & 0.090 & $0.016(-)$ \\
\hline 101 & 11 & 0.120 & 0.080 & $0.031(+)$ \\
\hline $101 \mathrm{~A}$ & 12 & 0.100 & 0.190 & $0.004(+)$ \\
\hline 102 & 10 & 0.210 & 0.100 & $0.011(-)$ \\
\hline 103 & 12 & 0.200 & 0.120 & $0.068(+)$ \\
\hline 104 & 6 & 0.140 & 0.010 & $0.061(+)$ \\
\hline 105 & 33 & 0.400 & 0.170 & $0.072(+)$ \\
\hline 106 & 11 & 0.020 & 0.350 & $0.131(-)$ \\
\hline Average & & & & $0.016(+)$ \\
\hline
\end{tabular}

${ }^{a}$ Based on one set of wet-bulk density values per section of core. A set consists of the average GRAPE value per section and a value derived from one shipboard sample per section.

${ }^{b}$ Maximum amount by which GRAPE values exceed values derived from shipboard samples.

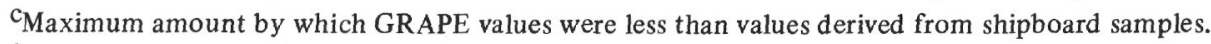

$\mathrm{d}_{(+)}=$GRAPE site average exceeds site average for shipboard samples.

$(-)=$ GRAPE site average less than site average for shipboard samples.

It should be understood that the values listed as "upper" and "lower" limits represent extremes; for example, only one GRAPE value out of 33 from Site 105 deviated by $0.40 \mathrm{~g} / \mathrm{cc}$ whereas the next highest deviation was $0.24 \mathrm{~g} / \mathrm{cc}$. At

Site 102 , only one GRAPE value deviated from the shipboard value as by much as $0.21 \mathrm{~g} / \mathrm{cc}$, whereas, all other deviations were generally less than $0.10 \mathrm{~g} / \mathrm{cc}$. At Site 106 , only two GRAPE values differed by amounts greater than $0.17 \mathrm{~g} / \mathrm{cc}$. Statistical analysis showed that 90 per cent of the Leg 11 GRAPE values ranged from $0.17 \mathrm{~g} / \mathrm{cc}$ less than shipboard sample values to $0.19 \mathrm{~g} / \mathrm{cc}$ greater than shipboard sample values for an average difference of $0.01 \mathrm{~g} / \mathrm{cc}$ by which GRAPE values exceeded shipboard sample values.

TABLE 9

Distribution of Sound Velocity Measurements for Leg 11 Cores

\begin{tabular}{lcccr}
\hline Hole & $\begin{array}{c}\text { Total Cores } \\
\text { Recovered }\end{array}$ & $\begin{array}{c}\text { Total Cores } \\
\text { Measured }\end{array}$ & $\begin{array}{c}\text { Maximum Depth } \\
\text { Velocity Data }\end{array}$ & Hole \\
\hline 98 & 15 & 11 & 241 & 357 \\
$99 \mathrm{~A}$ & 14 & 2 & 22 & 248 \\
100 & 13 & 1 & 210 & 331 \\
$101-101 \mathrm{~A}$ & 12 & 5 & 197 & 691 \\
102 & 19 & (none) & --- & 661 \\
103 & 7 & 1 & 8 & 449 \\
104 & 10 & 10 & 9 & 617 \\
105 & 43 & 1 & $368^{\mathrm{b}}$ & 633 \\
106 & 14 & (none) & $-\ldots-$ & 78 \\
107 & 1 & (none) & --- & 209 \\
108 & 2 & & & \\
\hline
\end{tabular}

aSub-seafloor depths in meters for the deepest velocity measurement and for the total depth of the bore hole.

${ }^{b}$ Deepest measurement was on Core 19 at 435 meters. Core 19 should be discarded since entire core consists of hole cavings. 


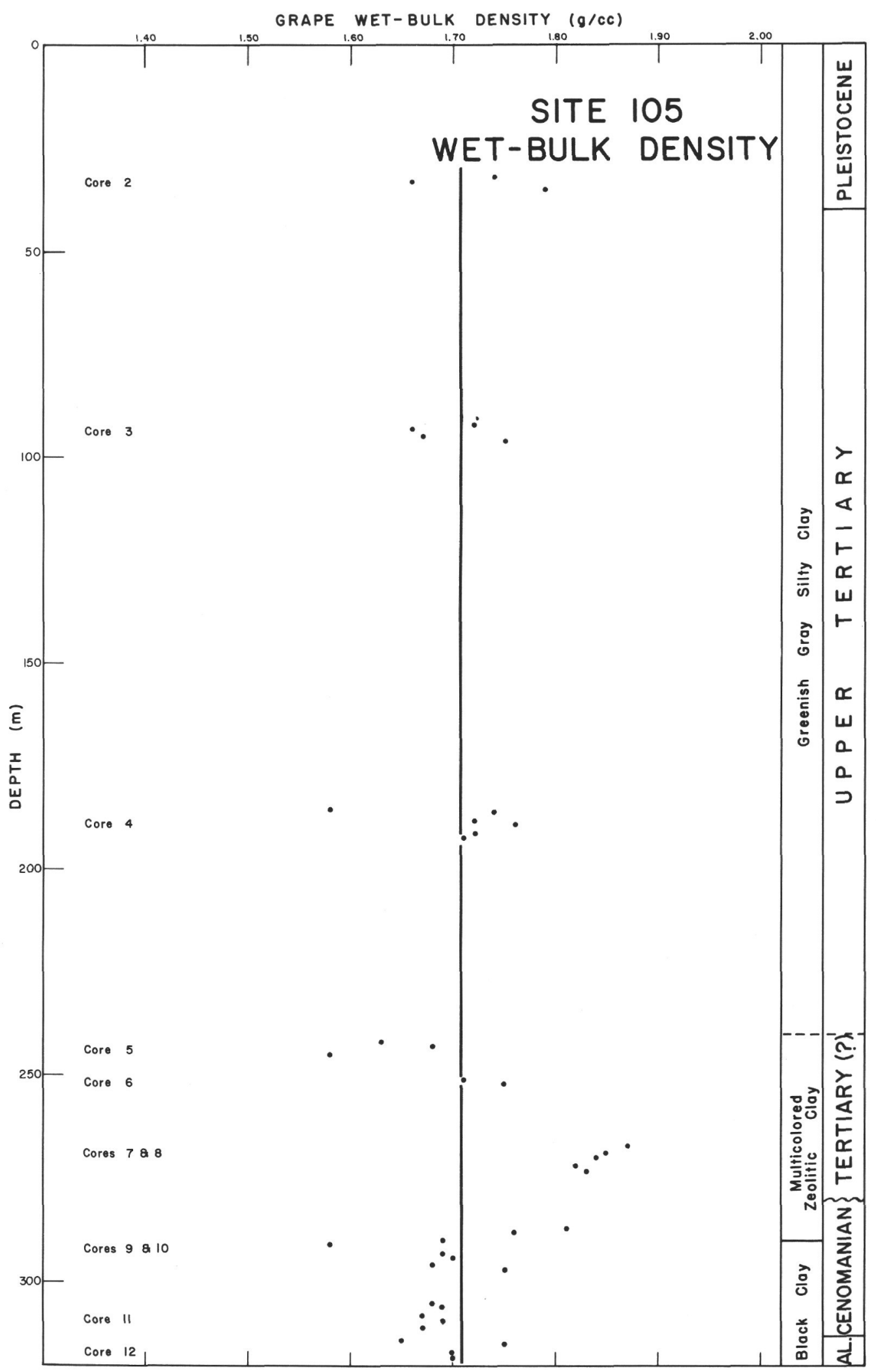

Figure 13. Wet-bulk density and depth crossplot for silty clays at Site 105. Each point represents the average GRAPE wet-bulk density per section of core. Wet-bulk densities derived from shipboard samples showed, in general, more scatter but a similar vertical trend for the average wet-bulk density curve. 
The pulse method is described in detail by Winokur and Chanesman (1966), and by Boyce (in Chapter 2, Maxwell et al., 1970) with respect to equipment, techniques, and measurement accuracy applicable to the DSDP cruises.

\section{Velocity-Depth Crossplots}

It has been established that, in general, there is little change in velocity between 90 per cent porosity down to about 75 per cent porosity, and that most of these high porosity sediments have sound velocities less than in sea water (Hamilton, 1970). Below about 75 per cent porosity the familiar inverse relationship develops with velocity increasing as porosity decreases. A comparison of the velocity-depth crossplots with the porositydepth crossplots for Sites 98 and 105 (Figures 9 and 10 with Figures and 10) demonstrates this inverse relationship.

The average velocity curve from the crossplot for the carbonate sediments at Site 98 shows that velocities range from about $1550 \mathrm{~m} / \mathrm{sec}$ at 5 meters to about $1580 \mathrm{~m} / \mathrm{sec}$ at 25 meters. Below this depth the velocity remained fairly constant ( 1590 to $1600 \mathrm{~m} / \mathrm{sec}$ ) until a depth was reached at which recrystallization of the biocalcite had progressed to the point where the carbonate ooze began to exhibit the characteristics of a limestone. This depth appears to have been reached at Site 98 in Core 12 at a sub-seafloor depth of 240 meters. Core 12 recovered the first hard micritic chalk which marks the top of the Paleocene and corresponds with the top of a strong sequence of seismic reflectors noted at 0.25 seconds on the shipboard seismic profile. As noted in the Site Report for Site 98 (this volume), the correlation of the reflector at 0.25 seconds with the top of the hard Paleocene chalks would indicate an average velocity of $1920 \mathrm{~m} / \mathrm{sec}$, which is about $300 \mathrm{~m} / \mathrm{sec}$ faster than what is indicated by the velocity-depth crossplot in Figure 14. It must be remembered that the velocity values used for the crossplot have not been corrected for the effects of temperature and pressure at in situ conditions, whereas the seismic profile data includes the effect of these conditions.

The average velocity curve derived from the velocity-depth crossplot for Site 105 (Figure 15) shows the velocity ranging from about $1540 \mathrm{~m} / \mathrm{sec}$ at 90 meters to about $1660 \mathrm{~m} / \mathrm{sec}$ at 310 meters. Intervening measurements suggest a fairly uniform rate of increase in velocity with depth. Although the porosity-velocity relationship appears normal for the silty clays at Site 105 , the rates at which these two parameters change with depth are such as to suggest the presence of a sediment consolidation anomaly. A comparison of these rates can be made by calculating a compaction correction for various levels in the unconsolidated silty clays. The compaction correction trend for Site 105 is shown in Table 13. The trend is unusual in that the compaction correction factor remains constant with increasing depth. A more normal compaction correction trend is shown by the calculation of this factor from the porosity and velocity curves (Figures 8 and 14) for Site 98 where the factor decreases with depth (Table 13). The abnormal compaction correction trend for the silty clays at Site 105 suggests the presence of a sediment consolidation anomaly in these clays. The anomaly is discussed more fully in the Sediment Consolidation section of this chapter.

\section{Leg 11 Velocity Plots \\ (Physical Property Pages) \\ Velocity-Penetrometer Relationship}

As the clay sediments become more compacted with depth they become less porous and develop a firmness which greatly reduces depth of penetration by the penetrometer needle. As porosity is reduced velocity should increase. Therefore, as the penetrometer plot migrates toward the right with depth in column 2 of the physical property pages, there should be a tendency for a corresponding migration toward higher velocities (toward the right) in column 6. This correlation, although subtle, can be detected on the core scale physical property pages for Hole 101 (Core 2), Hole 101A (Core 2), and Hole 105 (Core 4) (Site Reports, this volume).

\section{Velocity-Grain Size Relationship}

The relationship between grain size and sound velocity in shallow sediments has been studied by Horn et al. (1968), Schreiber (1968), Hamilton (1970), and others. For deep sediments (below about 1500 meters) grain size has little or no influence on velocity. The velocity in these deep sediments is primarily a function of pore volume and the manner in which clay is distributed in the sediment. Hamilton (1970) concluded that data derived from laboratory analysis of sediment size have been found to be among the best indices to empirical derivation of sound velocity in shallow sediments. He states (p. 4437) that:

Grain sizes affect velocity through their effects on porosity, density, and other factors. As index properties to sound velocity, percentages of sand, silt, and clay are as important as mean grain size, and better than porosity and density in all the environments. In continental terrace sediments, per cent sand and mean grain size are about equal value in deriving velocity. In abyssal hill and abyssal plain sediments, per cent clay (or its complement, per cent silt plus per cent sand) may be slightly better than mean grain size in deriving velocity. 


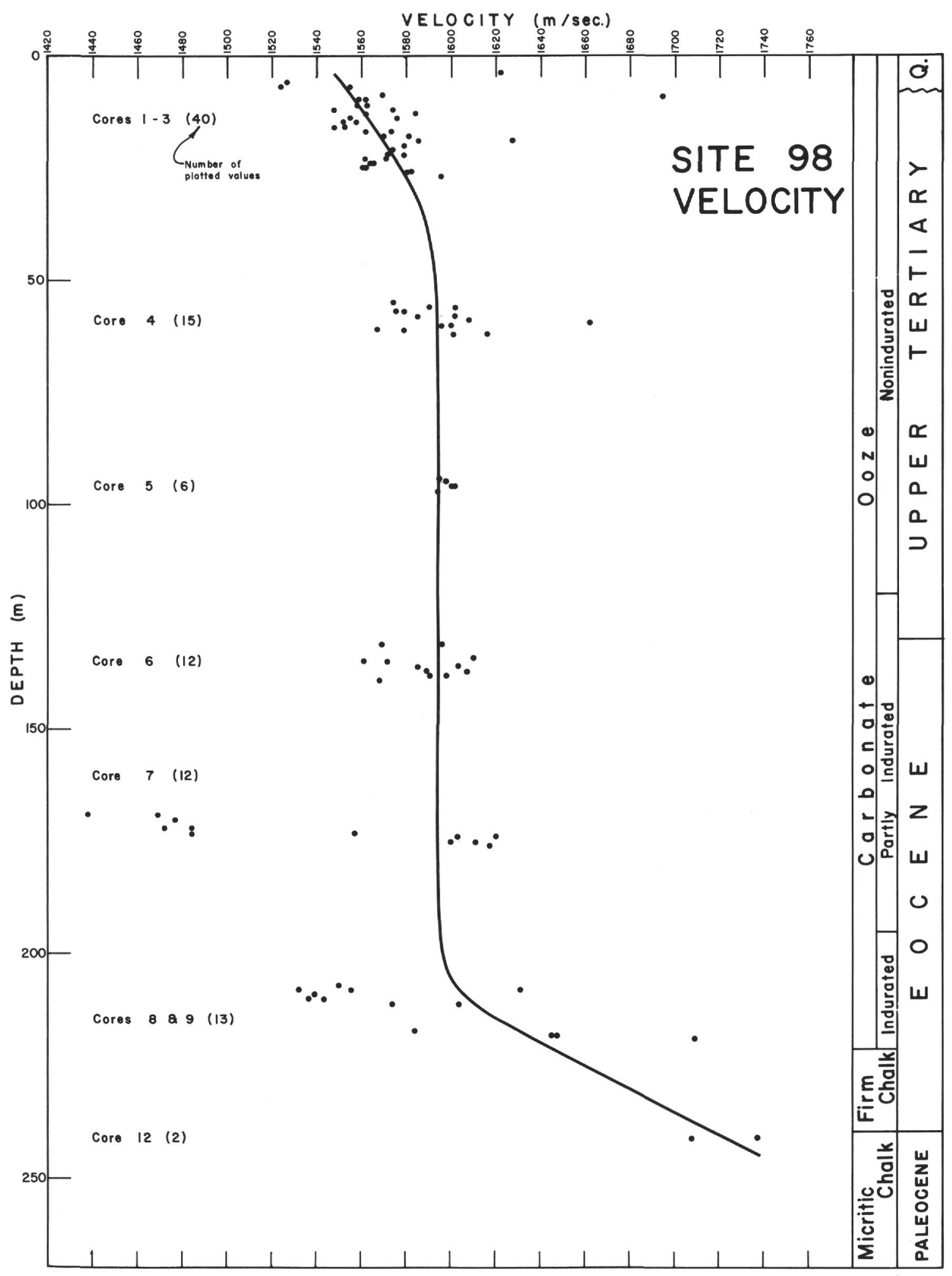

Figure 14. Velocity-depth crossplot for carbonate sediments at Site 98. All values are measurements made at ambient laboratory temperature and atmospheric pressure and are therefore not representative of in situ velocities. Interstitial water salinity averaged 34,800 ppm (Section IX, this Chapter). Each point represents a single velocity measurement at a specific point within the core. Three low values (1310-1318) in core 4-6 were not used. Six low values $(1438-1484)$ in core $7-(2,4)$ are in highly disturbed sediment. Two low values in core II were in highly disturbed sediment and omitted from the plot. All excessively low values are plotted in column 6 of the physical property pages of the Site Report for Site 98. 


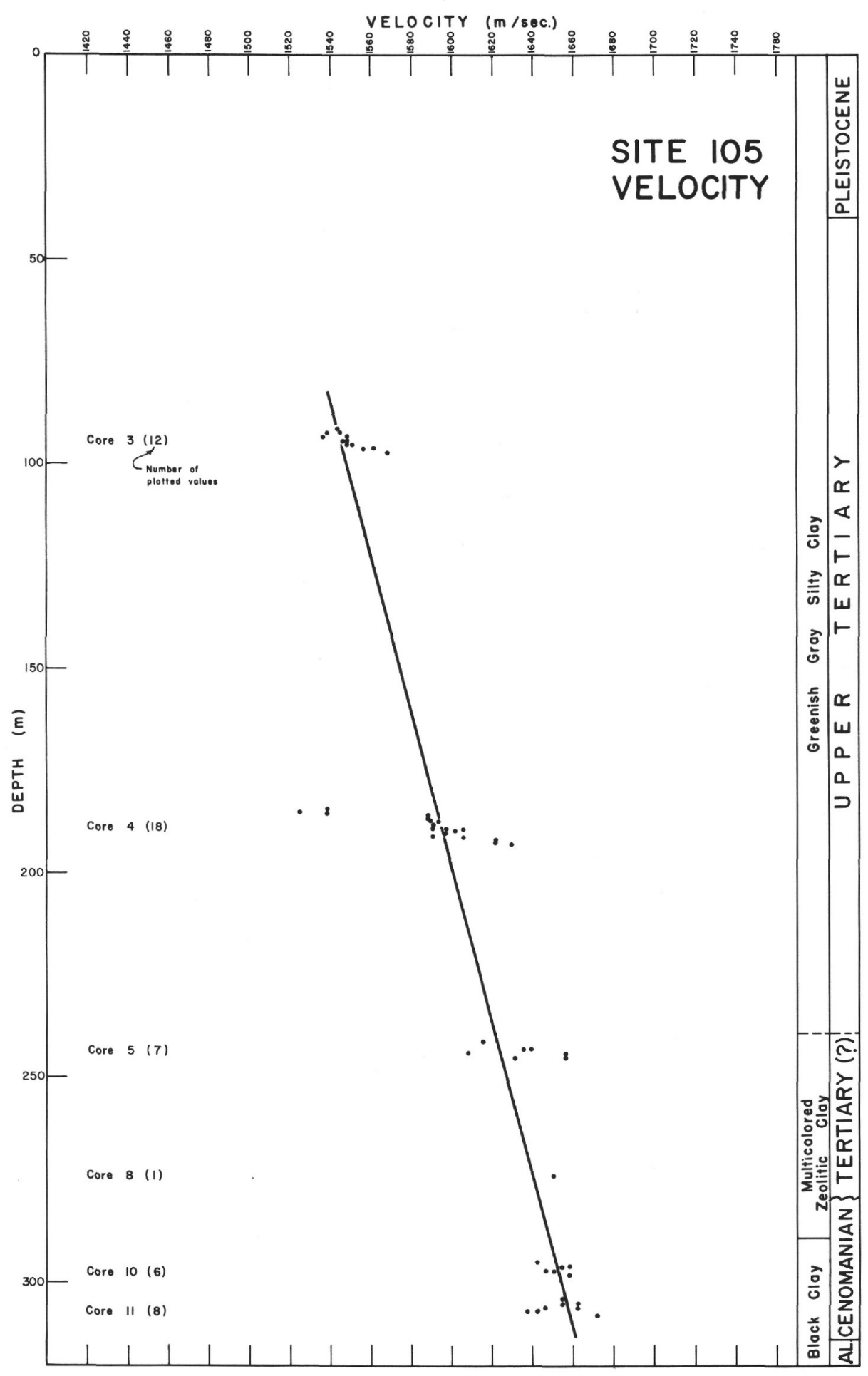

Figure 15. Velocity-depth curve for silty clays at Site 105. All values are measurements made at ambient laboratory temperature and atmospheric pressure and are therefore not representative of in situ velocities. Interstitial water salinity averaged 32,899 ppm (Section IX, this Chapter). Each point represents a single velocity measurement at a specific point within the core. Cores 8-(5, 6). 13, and 19 contained hole cavings and all values in these intervals were omitted. A single value in core 15 was in highly disturbed sediment and omitted. All values omitted from this crossplot are plotted in column 6 of the physical property pages of the Site Report for Site 105. 
Hamilton illustrated the empirical relationship between velocity and each of the three grain-size parameters (per cent clay, per cent sand, and mean grain diameter) with crossplots. Although he drew no average curves through the crossplots, velocity trends are readily discernible. An average straight line curve can be drawn through the scattered points shown in his crossplot of velocity and per cent clay. Based on this curve it appears that a 20 per cent increase in the amount of clay would decrease the velocity by about $25 \mathrm{~m} / \mathrm{sec}$. Because Leg 11 grain-size analyses generally were not made at the exact depth of the velocity measurement and because the velocity measurements often varied by more than $25 \mathrm{~m} / \mathrm{sec}$ within an apparently homogeneous sediment, it would be difficult to establish from Leg 11 data a verification of Hamilton's empirical relationship between velocity and per cent clay. A 20 per cent variation in the amount of clay can be readily detected by visual examination of the grain-size plots shown in column 3 of the physical property pages in the Site Reports of this volume; however, because of the scale used on the physical property pages, a $25 \mathrm{~m} / \mathrm{sec}$ deflection in the velocity plot amounts to only a fourth of the deflection caused by a 20 per cent change in the amount of clay and is therefore not readily discernible by visual examination. A visual comparison of the velocity and grain size plots in columns 3 and 6 of the physical property pages revealed no readily apparent correlations.

\section{Velocity as an Indicator of \\ Sediment Type and Environment}

Accumulation of basic data necessary for the understanding of the relationships between sediment sound velocity and other physical properties of marine sediments has been accelerating since the early 1950's. For a brief history of compressionalwave velocity studies in marine sediments the reader is referred to Hamilton (1970). According to Hamilton $(1970,1971)$ there is now sufficient information on hand to allow the prediction of an average velocity value for a given environment and sediment type. There is question, however, as to whether these empirical relationships would exist under in situ conditions.

Hamilton's measurements were made on the upper 30 centimeters of sediment from three major physiographic provinces of the North Pacific. Depths for Leg 11 velocity measurements ranged from near the sea floor to about 435 meters (Table 9). Only three velocity measurements were made on Leg 11 cores at a depth comparable to Hamilton's sampling, and which are in close proximity to Leg 11 grainsize analyses. Data for these measurements are listed in Table 10. Table 11 compares the Leg 11 velocities and lithologies listed in Table 10 with the average velocities for each of the two environments discussed by Hamilton.

Measurement of sedimentary temperature at the time of the velocity measurement is critically important when comparing velocities of one sedimentary environment with those of a different environment because, as stated by Hamilton (1970, p 4425):

....variations in 'room temperature' can cause changes in sediment velocity on the order of 20 to $30 \mathrm{~m} / \mathrm{sec}$ which can be more than some variations between sediment types or environments.

Because of the temperature variation effect, caution should be used in drawing conclusions from a comparison of Leg 11 velocities with those of Hamilton's listed in Table 11.

\section{Summary}

Leg 11 sediment sound velocities are reported at ambient laboratory temperature and pressure. Almost all of the velocities were measured through disturbed sediment samples, thus they could not accurately represent in situ conditions even after temperature and pressure corrections. However, they are still of interest as they may be used to construct a velocity-depth crossplot from which can be

TABLE 10

Leg 11 Core Samples with Both Grain-Size and Velocity Data less than 75 centimeters Sub-Seafloor

\begin{tabular}{|c|c|c|c|c|c|c|c|}
\hline \multirow{2}{*}{$\begin{array}{l}\text { Sample Designation } \\
\text { (Site-Core-Section) }\end{array}$} & \multicolumn{2}{|c|}{ Depth Below Seafloor $(\mathrm{cm})$} & \multicolumn{3}{|c|}{ Grain Size $(\%)$} & \multirow[b]{2}{*}{ Lithology } & \multirow{2}{*}{$\begin{array}{l}\text { Velocity } \\
(\mathrm{m} / \mathrm{sec} .)\end{array}$} \\
\hline & (Grain-size Data) & (Velocity Data) & Sand & Silt & Clay & & \\
\hline $99-1-1$ & 37 & 38 & 13.5 & 26.1 & 60.4 & Silty clay & 1507 \\
\hline $103-1-1$ & 8 & 38 & 19.5 & 33.3 & 47.0 & Silty clay & 1516 \\
\hline $104-1-1$ & 10 & 38 & 44.5 & 25.8 & 29.7 & Sand-silt-clay & 1523 \\
\hline
\end{tabular}


TABLE 11

Silty Clay Velocities for Near-Seafloor Sediments in Abyssal Environments

\begin{tabular}{lcc}
\hline \multicolumn{1}{c}{ Environment } & $\begin{array}{c}\text { Abyssal Hill } \\
\text { (pelagic) }\end{array}$ & $\begin{array}{c}\text { Abyssal Plain } \\
\text { (turbidite) }\end{array}$ \\
\hline $\begin{array}{l}\text { Hamilton's Velocities } \\
(\mathrm{m} / \mathrm{sec})\end{array}$ & 1507 & 1521 \\
& & \\
$\begin{array}{l}\text { Leg 11 Velocities } \\
(\mathrm{m} / \mathrm{sec})\end{array}$ & 1516 & 1507 \\
\hline
\end{tabular}

NOTE: Pore water salinity applies in all velocity measurements. Hamilton's velocities corrected to $23^{\circ}, 1$ atmosphere. Leg 11 velocities measured at ambient laboratory temperature and atmospheric pressure.

derived an average velocity curve which may be considered representative of the velocity trend throughout the interval in which the velocity measurements are located. The average velocity curve can be used in conjunction with the average porosity curve to determine the compaction correction trend for use in sediment consolidation studies.

\section{SEDIMENT CONSOLIDATION}

Consolidation of sediments includes many factors. For clays, a primary factor is compaction. There are many uncertainties involved in the study of the complex process of compaction. Weller (1959, p. 279) categorized the uncertainties as follows:

(1) the compositional nature of sediment, particularly its mineralogy and the size distribution and shapes of its constituent particles; (2) the properties and influences of water films and internal water; (3) the effects of factors varying with depth such as temperature, pressure, and the composition of brines which determine the viscosity, density, and chemical activity of water; (4) the time required for sediments to reach equilibrium after burial.

Although no formal studies of sediment consolidation per se were conducted during Leg 11 , measurements were made which show the influences of certain consolidation factors such as compaction and carbonate diagenesis. Data relating to these factors were derived from the following: (1) shipboard penetrometer measurements, (2) shipboard measurements of porosity and velocity, and (3) shipboard petrographic analyses. Although this chapter includes separate sections on porosity and velocity, these two parameters together with the penetrometer data will be discussed here in the Sediment Consolidation section to the extent that their measurements can be used to illustrate the processes of consolidation.
At the upper limit the water films absorbed on sedimentary particles come into contact and at the lower limit the water films have been squeezed out from between these particles and contact is established between the mineral grains themselves.

The average porosity curve for Site 102 shows that at the depth of about 125 to 130 meters the clay porosities are approaching the lower limit of Weller's 80 to 45 per cent range. It is assumed, therefore, that at this depth, clay mineral grains begin to make contact with each other to the extent that there is a marked increase in sediment strength which tends to inhibit compaction at greater depths.

The average porosity curve for the silty clays at Site 106 (Figure 11) also reflects the influence of the marked change in the rate of consolidation which occurs in most of Leg 11 core holes at about the 125-meter depth. Both sets of porosity data (shipboard sample and GRAPE) were plotted for Site 106. Each average porosity curve shows a relatively rapid rate of decrease in porosity down to about 120 meters. GRAPE data, available down to 958 meters, shows that compaction proceeds at a much slower rate for sediments below the 120meter level. The more rapid rate of consolidation occurs within the rate of 68 to 51 per cent (shipboard sample porosity). This is comparable to the 76 to 53 per cent range for the first 130 meters of sediment at Site 102 .

It is proposed that the marked change in the slope of the average porosity curves at 120 to 130 meters at Sites 102 and 106 reflects the effects of the water film phenomenon which Weller described for the clay porosity range of 80 to 45 per cent, Table 12 compares the Leg 11 shipboard sample porosity ranges for the first 120 to 130 meters at Sites 102 and 106 with Weller's data.

Weller's depth range in Table 12 is based on his graphs of porosity-depth curves (Weller, 1959, p. 276). His discussion which preceded the graphs pointed out that, at the time, the depth relations of porosities in excess of 35 per cent had not received

Table 12. Depth and Porosity Ranges

\begin{tabular}{lcc}
\hline & $\begin{array}{c}\text { Depth } \\
\text { Range } \\
(\mathrm{m})\end{array}$ & $\begin{array}{c}\text { Shipboard Sample } \\
\text { Porosity Range } \\
(\%)\end{array}$ \\
\hline Weller (1959) & $0-30$ & $80-45$ \\
Site 102 & $4-120$ & $76-53$ \\
Site 106 & $2-130$ & $68-51$ \\
\hline
\end{tabular}




\section{Penetrometer Measurements}

Penetrometer measurements indicate to what depth a standard needle will penetrate the smooth surface of a split core. The direction of penetration is parallel to the depositional surface. The standard needle operates under a fixed load of 50 grams $( \pm 0.1$ gram). It is 1.00 to 1.02 millimeters in diameter and about 5 centimeters in length. The instrument is known as the AP-210 penetrometer (Soil Test, Inc.). The penetrometer test follows the "Standard method of test for penetration of bituminous materials" (ASTM, 1965).

The penetrometer measurements were recorded in tenths of millimeters. Penetration tests were made generally at three levels in each 1.5-meter core section. Three readings were taken in separate spots at each level and averaged. These averages form the basis for the penetrometer plot shown in column 2 of the physical property pages in the Site Reports (this volume).

Specific relationships between penetrability and other engineering tests have not yet been established. Although the measurements do not represent a specific value of sediment strength, they can, however, be used as a basis for comparing relative degrees of consolidation. In general, depth plots of Leg 11 penetrometer measurements show a relatively rapid rate of sediment consolidation for about the first 125 meters of hole penetration. Below this depth, sediment consolidation appears to proceed at a considerably slower rate. This consolidation phenomenon is illustrated by the penetrometer curves in the Site Summary Charts (Figure 3, Site Reports, this volume).

\section{Porosity and Wet-Bulk Density}

\section{Clay Sediments}

The marked change in the rate of sediment consolidation at about 125 meters should be reflected in the porosity-depth curves, since the porosity parameter is so closely related to sediment compaction. The average porosity curve, based on shipboard sample porosities for the silty clays at Site 102 (Figure 9), shows a relatively rapid rate of decrease from an average of about 76 per cent at 4 meters below the sea floor to an average of about 53 per cent at 130 meters. Below 130 meters the porosity continues to decrease but at a much slower rate until it averages about 42 per cent at 661 meters (total depth of core hole). The marked lessening at 125 to 130 meters in the rate of decrease in porosity is most likely related to the water film phenomenon associated with Weller's porosity range of 80 to 45 per cent. Weller (1959, p.282) comments on this range thusly:

adequate investigation because sediments in this range were generally not consolidated sufficiently to be cored by the usual oil field methods. Therefore, in the higher range of porosities, Weller used the results of laboratory compression tests by Terzaghi (1925) who published a graph showing the relations of void ratio to pressure. Weller converted these data to develop approximate relations of porosity and depth. It would appear from the initial investigation of Leg 11 data that it may be necessary to revise Weller's porosity-depth curve in the higher ranges of clay porosities in order for it correspond more closely to the data which are being obtained from the ocean sediments recovered by the Deep Sea Drilling Project.

The average porosity curve for silty clay at Site 105 shows the expected overall relationship wherein porosity decreases with depth, but does not show the sediment consolidation phenomenon which occurs at 125 to 130 meters at Sites 102 and 106. The apparent absence of this phenomenon may be due in part to the sparsity of porosity data, the expanded vertical scale as compared to Figures 9 and 11 , or some unusual events in the history of sedimentation and sediment diagenesis at Site 105. The following Velocity section (part of Sedirzent Consolidation) describes a consolidation anomaly which appears to be present at Site 105 and which may be related, at least in part, to the unusual metal enrichment of the multicolored clays in Cores 5 through 9 (Figure 16).

A crossplot of wet-bulk density and depth was made for Site 105 (Figure 13). Both sets of data (GRAPE and shipboard sample) were plotted originally; however, since the shipboard sample data showed, in general, a wider scatter of plots than did the GRAPE data, it was decided to show only the GRAPE plots in Figure 13. The wet-bulk density relations seem to be more irregular than would normally be expected. This irregularity is probably caused by the influence of varying densities of different mineral associations. This suspected causal relationship is based on the fact that the silty clays at Site 105 contain a zone of unusual mineralization at a depth of about 180 meters to about 300 meters (Figure 16). Metal enrichment of the multicolored clays in this zone are discussed elsewhere in this volume (Lancelot, et al., Chapter 31). The failure of the average wet-bulk density curve in Figure 13 to show the normally expected increase with depth tends to confirm the presence of a consolidation anomaly in the silty clays at Site 105. Further confirmation of this anomaly is presented here in the Sound Velocity section under Sediment Consolidation. 


\section{METAL ENRICHED \\ AND \\ MULTICOLORED CLAY ZONES SITE 105}

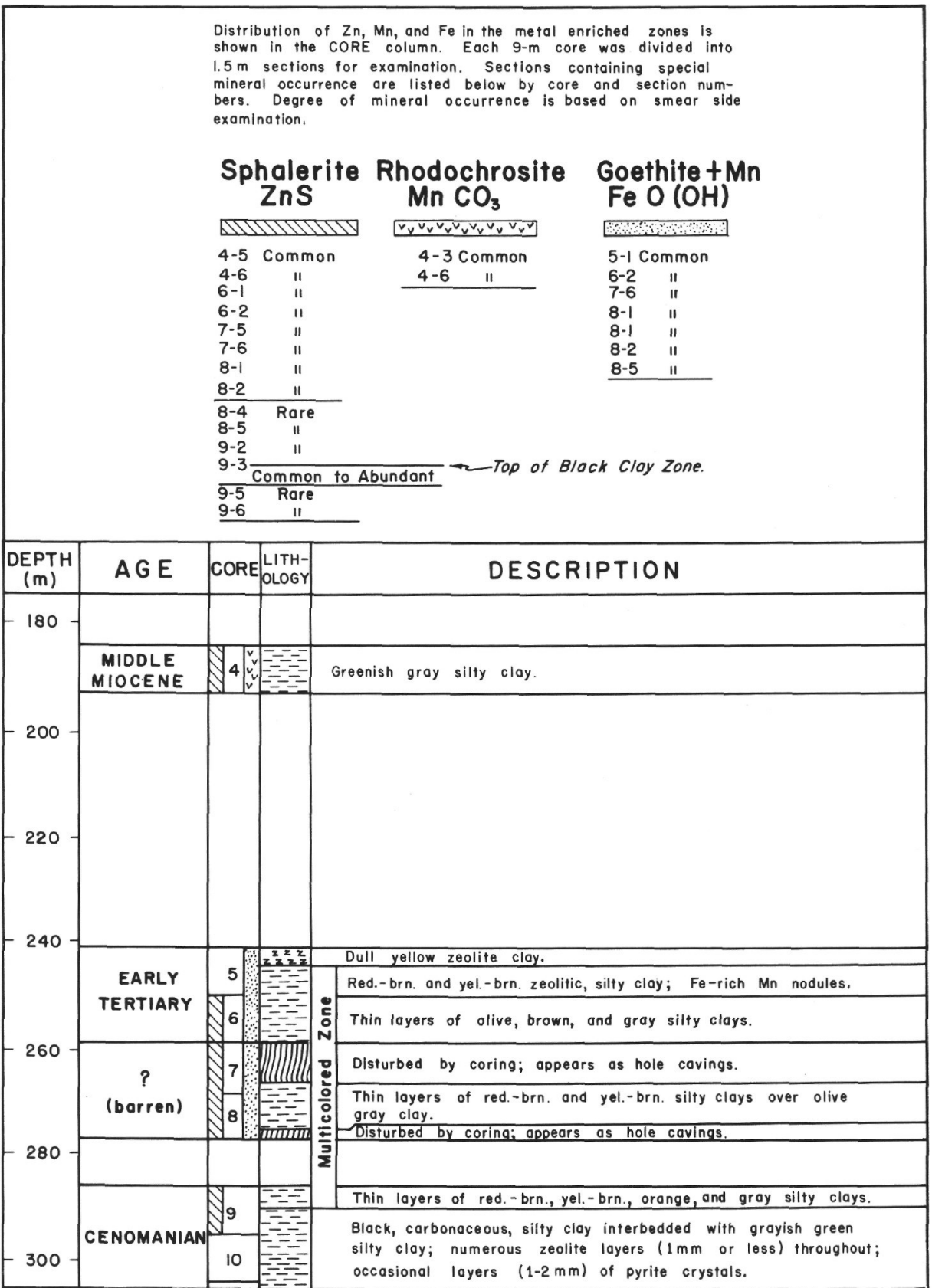

Figure 16. This section of core hole log for Site 105 shows the distribution of some unusual mineral occurrences associated with both the Tertiary-Cretaceous boundary and the middle Miocene. Some of the irregular density-depth relations shown in Figure 13 are attributed, in part, to the influence of varying densities for these mineral associations. 


\section{Carbonate Sediments}

Because carbonate muds compact less readily than clays (Terzaghi, 1940) and because carbonate diagenesis complicates the carbonate compaction process in many ways (recrystallization, cementation, aragonite conversion, etc.), it is not surprising that the porosity and velocity curves for the carbonate oozes at Site 98 (Figures 8 and 14) show compaction trends which are somewhat different from those for the silty clays at Sites 102, 105 and 106.

The average GRAPE porosity curve for the carbonate sediments at Site 98 shows porosity decreasing rapidly from about 68 per cent at 1 meter to about 59 per cent at 25 meters. Below 25 to 35 meters the average porosity curve indicates no regular change in porosity with depth until at about 220 meters the curve shows a relatively rapid rate of decreasing porosity. Much of the consolidation of the calcareous ooze seems to have been accomplished by the time 25 meters of overburden accumulated. This is in agreement with the statement of Weller (1959, p. 298) wherein he concluded, after noting the lack of tighter grain packing and the occurrence of uncrushed empty shells and porous structures in samples of limestone rock, that:

....consolidation of limestone was accomplished at an early stage before it was subjected to the pressure of much overburden.

Carbonate diagenesis appears to have been the controlling factor in the consolidation of the sediments below about 25 to 35 meters at Site 98 . The average porosity curve shows no variation in porosity until a depth is reached at which recrystallization of calcareous nannoplankton and other calcitic debris has progressed to the stage where the original calcareous oozes and chalks begin to exhibit the characteristics of a crystalline limestone. The incipient stages of this state of recrystallization were first seen in the firm chalks in Core 10 at 227 meters. At 240 meters recrystallization appears to be reaching the stage which characterizes finegrained limestone as evidenced by the recovery of hard micritic chalk in Core 12. The recrystallized nanno calcite $^{1}$ first appeared in the sediments in Core 6 (Site Report, this volume).

\section{Sound Velocity}

\section{Clay}

Only two Leg 11 core holes have shipboard velocity data deeper than 210 meters. The velocity measurements for one of these holes (Site 105) were in silty

\footnotetext{
${ }^{1}$ Recrystallized nanno calcite refers to calcite material of the size of nannoplankton or nannoplankton debris which shows no evidence of organic structure, but probably represents crystallization of calcareous nannoplankton (See report for Site 98 , this volume).
}

clays while measurements in the other hole (Site 98) were in carbonates. The average velocity curve for the silty clays at Site 105 shows the usual overall influence expected from the compaction of unconsolidated clay sediments (Figure 15). In addition to showing the expected depth relationship, the curve can also be used for the derivation of a compaction correction, an indice which is used in oil exploration when it is necessary to make acoustic log calculations of porosities in shallow unconsolidated formations. Compaction corrections were calculated at four levels for the silty clays at Site 105 in an attempt to obtain further confirmation of the consolidation anomaly which is suspected of being present at this site. Results of the compaction correction calculations are listed in Table 13. These values would normally decrease with depth but here they remain constant, which is generally an indication that compaction is not increasing with depth. The abnormal trend of compaction correction tends to confirm the presence of a consolidation anomaly in the silty clays at Site 105 .

In retrospect, it has been demonstrated that the porosity curve (Figure 10) and velocity curve (Figure 15) for the silty clays at Site 105 show the normal depth relationships. Wet-bulk densities and compaction corrections do not respond to depth in a normal fashion, and this abnormality is considered indicative of a consolidation anomaly in the silty clays at Site 105. Because the porosity and velocity curves suggest that compaction has progressed in a relatively normal manner at Site 105, it is necessary to investigate other consolidation factors in an attempt to develop an explanation for the consolidation anomaly. Such an investigation is beyond the scope of this report; however, it is suggested that unusual events in the history of sedimentation and of sediment diagenesis may be responsible for the consolidation anomaly. These events would include: (1) a possible $30 \pm$ million year hiatus in the sedimentary record at Site 105, and (2) the metal enrichment of the multicolored clays associated with the hiatus (Figure 16) (see Site reports and chapter by Lancelot et al., this volume).

\section{Carbonates}

A comparison of the average velocity curve for the pelagic carbonate sediments at Site 98 (Figure 14) with the average porosity curve for the same site shows clearly the inverse relationship between porosity and velocity. Analysis of the velocity curve at this site confirms what has already been said about carbonate consolidation (under Carbonate Sediments above), that is carbonate diagenesis rather than compaction is the primary factor in the consolidation of carbonate sediments. This is shown by the fact that, except for the effects of the initial consolidation stage at 0 to 25 meters, the curve shows no change with depth until the 
level is reached at which calcareous nannoplankton and other calcitic debris have become sufficiently recrystallized to form the indurated oozes and firm chalks at the 200 to 220-meter level (Figure 14). Recrystallization has proceeded to a more advanced stage in Core 12 at 240 meters where the highest (and deepest) velocities were recorded in micritic chalks. These hard chalk beds mark the top of the Paleocene and correspond with the top of a strong sequence of seismic reflectors which are discussed in the Sound Velocity section of this chapter and in the Site Report for Site 98 (this volume).

Compaction corrections were calculated for the carbonate sediments at Site 98 . The results of these calculations are listed in Table 13. The compaction correction trend is a normal one increasing with depth. The increase is slight but this is as it should be for carbonate sediments where compaction is generally not the major factor in the consolidation history of the sediments.

TABLE 13

Compaction Corrections for Sites 98 and 105

\begin{tabular}{rcrc}
\hline \multicolumn{2}{c}{ Site 98} & \multicolumn{2}{c}{ Site 105} \\
$\begin{array}{c}\text { Depth } \\
(\mathrm{m})\end{array}$ & $\begin{array}{c}\text { Compaction } \\
\text { Correction }\end{array}$ & $\begin{array}{c}\text { Depth } \\
(\mathrm{m})\end{array}$ & $\begin{array}{c}\text { Compaction } \\
\text { Correction }\end{array}$ \\
\hline 0 & 0.158 & 90 & 0.131 \\
10 & 0.146 & 150 & \\
20 & 0.141 & 150 & 0.131 \\
50 & 0.142 & & \\
220 & 0.144 & 200 & 0.131 \\
230 & 0.140 & & \\
240 & 0.133 & 300 & 0.131 \\
\hline
\end{tabular}

\section{INTERSTITIAL WATERS}

Shipboard measurements of salinity, $p \mathrm{H}$, and Eh were made on the interstitial waters taken from 41 sediment samples representing nine of the eleven drill sites for Leg 11. Sampling frequency, never more than one per core, ranged from 1 to 12 per drill site as shown in Table 14. Sub-seafloor sampling depths ranged from 1.5 meters at Site 106 to 618 meters at Site 102 .

Water samples were obtained on board by extruding the core sample into the barrel of a specially designed passivated stainless steel squeezer which was placed in a hydraulic press and squeezed under 5000 to 8000 pounds pressure. During the squeezing the pore water passed into a plastic syringe forcing the plunger before it so that no gas phase was allowed to contact the fluid. The syringe was then capped with a millipore filtering attachment. Approximately 0.2 milliliter of the interstitial water sample was used for determination of salinity with a Goldberg hand refractometer. Another 0.2 milliliter of the water sample was used for gas chromatography. About 0.6 milliliter of the sample was introduced into a specially designed electrochemical system for measurement of $p \mathrm{H}$ and $\mathrm{Eh}$.

The shipboard data, especially $p \mathrm{H}$ and Eh, could be in error for several reasons and may not be adequate for accurate calculations involving these parameters. The data may, however, be used for comparative purposes and for the plotting of general trends. General observations of the salinity data are discussed below. The $p \mathrm{H}$ and Eh data are discussed elsewhere in this volume (Presley and Kaplan, Lancelot and Ewing).

The salinity range $(27,500$ to $35,200 \mathrm{ppm})$ had an average of $32,221 \mathrm{ppm}$ which is quite close to that of normal sea water $(30,000 \mathrm{ppm})$. The $p \mathrm{H}$ range ( 7.07 to 8.14 ) had an average of 7.6. Eh ranged from -0.002 to -0.061 and from +0.007 to +0.150 . Table 14 shows that thirty-three of the samples were from clay lithologies while eight came from carbonate ooze. The samples were mainly Tertiary in age with only two coming from pre-Tertiary sediments (ValanginianTithonian at Site 100 and middle Cretaceous at Site 105).

Salinities of interstitial waters from the clay sediments recovered on Leg 11 show a general tendency to decrease as the depth of sample increases. This trend is delineated by the shaded area in Figure 17. The trend is based on shipboard measurements from Sites 101 through 106. Some of the plots show a wide scatter in the deeper samples. It is believed that the variation may be due in part to the possibility that certain diagenetic effects may be more pronounced in the relatively older sediments. Lithification effects were suggested as a possible cause for the large salinity variations recorded in sediments from DSDP Site 21 (Maxwell, A. E. et al., 1970, p. 375).

The relationship between pore solution (interstitial water) salinity and depth of burial (degree of compaction) has been thoroughly discussed by Engelhardt and Gaida (1963, p. 922) who concluded that:

$$
\begin{aligned}
& \text {... compaction of very homogeneous clays can } \\
& \text { bring about shales containing nearly pure water. }
\end{aligned}
$$

The illustrated text of Engelhardt and Gaida (1963) explains the mechanism whereby pore solution salinity is decreased as compaction, or depth of burial, increases. They found that this relationship is true only for a lower pressure range of 30 to 800 atmospheres. Their work showed that the relationship reverses itself at pressures above 800 atmospheres. The reader is urged to consult the Engelhardt and Gaida paper for complete discussions of the pore solution salinitycompaction relationship and for a description of the pressure device which they used to simulate various 
TABLE 14

Shipboard Data on Interstitial Water and Gas Chromatography, Leg 11

\begin{tabular}{|c|c|c|c|c|c|c|c|c|c|c|c|c|c|c|}
\hline \multirow[b]{2}{*}{ Hole } & \multirow[b]{2}{*}{ Core-Section } & \multicolumn{13}{|c|}{ Gas Chromatographyc } \\
\hline & & $\begin{array}{l}\text { Deptha } \\
\text { (m) }\end{array}$ & $\begin{array}{l}\text { Temperature } \\
\left({ }^{\circ} \mathrm{C}\right)\end{array}$ & $\begin{array}{l}\text { Salinity } \\
\text { (ppm) }\end{array}$ & $\begin{array}{l}\text { Eh } \\
\text { (v) }\end{array}$ & $p \mathrm{H}$ & $\begin{array}{l}\text { "AIR" } \\
\%\end{array}$ & $\mathrm{CO}_{\%}$ & $\begin{array}{c}\mathrm{CH}_{4} \\
\%\end{array}$ & $\begin{array}{c}\mathrm{C}_{2} \mathrm{H}_{6} \\
\%\end{array}$ & $\begin{array}{c}\mathrm{H}_{2} \mathrm{~S} \\
\%\end{array}$ & Sediment Type & C.D. ${ }^{d}$ & Age \\
\hline \multirow[t]{7}{*}{98} & $1-6$ & 7 & 22.9 & 34,700 & -0.029 & 7.5 & & & & & & $\begin{array}{l}\text { Foraminiferal-nannofossil } \\
\text { ooze }\end{array}$ & 4 & Late Pleistocene \\
\hline & $3-5$ & 24 & 23.2 & 34,700 & -0.014 & 7.3 & & & & & & $\begin{array}{l}\text { Foraminiferal-nannofossil } \\
\text { ooze }\end{array}$ & 2 & Late Miocene \\
\hline & $4-6$ & 62 & 23.0 & 34,700 & -0.027 & 7.5 & & & & & & $\begin{array}{l}\text { Foraminiferal-nannofossil } \\
\text { ooze }\end{array}$ & 3 & Late Miocene \\
\hline & $5-3$ & 96 & 23.0 & 35,200 & -0.020 & 7.4 & & & & & & $\begin{array}{l}\text { Foraminiferal-nannofossil } \\
\text { ooze }\end{array}$ & 1 & Late Oligocene \\
\hline & $6-6$ & 137 & 23.2 & 34,700 & -0.019 & 7.3 & & & & & & $\begin{array}{l}\text { Foraminiferal-nannofossil } \\
\text { ooze }\end{array}$ & 1 & Late Eocene \\
\hline & $7-4$ & 173 & NR & 34,400 & -0.017 & 7.3 & & & & & & $\begin{array}{l}\text { Foraminiferal-nannofossil } \\
\text { ooze }\end{array}$ & 1 & Early Eocene \\
\hline & $11-2$ & 234 & 23.0 & 35,200 & -0.003 & 7.1 & & & & & & $\begin{array}{l}\text { Foraminiferal-nannofossil } \\
\text { ooze }\end{array}$ & 2 & Early Eocene \\
\hline $99 \mathrm{~A}$ & $1-4$ & 6 & NR & 35,200 & -0.017 & 7.4 & & & & & & Clay & 3 & Quaternary \\
\hline 100 & $1-4$ & 207 & 26.0 & 35,200 & -0.002 & 7.2 & & & & & & Nannofossil ooze & 1 & $\begin{array}{l}\text { Valanginian- } \\
\text { Tithonian }\end{array}$ \\
\hline \multirow[t]{2}{*}{101} & $1-5$ & 39 & 25.0 & 33,600 & -0.027 & 7.6 & & & & & & Hemipelagic mud & 1 & Early Pliocene \\
\hline & $2-3$ & 71 & 25.0 & 33,000 & -0.030 & 7.7 & & & & & & Hemipelagic mud & 2 & Late Miocene \\
\hline \multirow[t]{3}{*}{$101 \mathrm{~A}$} & $1-3$ & 118 & 25.2 & 33,000 & -0.027 & 7.6 & & & & & & Hemipelagic mud & 2 & Late Miocene \\
\hline & $2-5$ & 162 & 25.3 & 31,900 & -0.032 & 7.7 & & & & & & Hemipelagic mud & 1 & Late Miocene \\
\hline & $3-2$ & 247 & 25.6 & 32,500 & -0.027 & 7.6 & & & & & & Hemipelagic mud & 3 & Late Miocene \\
\hline \multirow[t]{8}{*}{102} & $1-5$ & 6 & 24.0 & 33,000 & NR & NR & & & & & & Hemipelagic mud & 2 & Quaternary \\
\hline & $2-5$ & 24 & 23.0 & 32,500 & -0.040 & 7.8 & & & & & & Hemipelagic mud & 2 & Late Pleistocene \\
\hline & $4-4$ & 100 & 23.0 & 31,900 & -0.048 & 7.9 & & & & & & Hemipelagic mud & 2 & Early Pleistocene \\
\hline & $5-5$ & 139 & 23.1 & 31,400 & -0.047 & 7.9 & 32.29 & 0.41 & 69.29 & & & Hemipelagic mud & 2 & Early Pleistocene \\
\hline & $7-5$ & 185 & 23.1 & 30,300 & -0.059 & 8.1 & & & & & & Hemipelagic mud & 2 & Early Pleistocene \\
\hline & $10-3$ & 309 & 23.5 & 29,700 & -0.058 & 8.1 & & & & & & Hemipelagic mud & 1 & Late Pliocene \\
\hline & $11-2$ & 354 & 24.0 & 29,200 & -0.060 & 8.1 & 52.59 & 0.40 & 47.01 & & & Hemipelagic mud & 1 & Late Pliocene \\
\hline & $12-4$ & 423 & 24.0 & 29,200 & -0.048 & 7.9 & 49.82 & 0.36 & 49.82 & & & Hemipelagic mud & 2 & Late Pliocene \\
\hline
\end{tabular}


TABLE 14 - Continued

\begin{tabular}{|c|c|c|c|c|c|c|c|c|c|c|c|c|c|c|}
\hline \multirow[b]{2}{*}{ Hole $\mathrm{C}$} & \multirow[b]{2}{*}{ Core-Section } & \multirow[b]{2}{*}{$\begin{array}{c}\text { Deptha }^{\mathrm{a}} \\
(\mathrm{m})\end{array}$} & \multirow[b]{2}{*}{$\begin{array}{c}\text { Temperature } \\
\left({ }^{\circ} \mathrm{C}\right)\end{array}$} & \multirow[b]{2}{*}{$\begin{array}{l}\text { Salinity } \\
\text { (ppm) }\end{array}$} & \multirow[b]{2}{*}{$\begin{array}{l}\text { Eh } \\
\text { (v) }\end{array}$} & \multirow[b]{2}{*}{$p \mathrm{H}$} & \multicolumn{4}{|c|}{ Gas Chromatographyc } & \multirow{2}{*}{$\begin{array}{c}\mathrm{H}_{2} \mathrm{~S} \\
\%\end{array}$} & \multirow[b]{2}{*}{ Sediment Type } & \multirow[b]{2}{*}{ C.D. ${ }^{d}$} & \multirow[b]{2}{*}{ Age } \\
\hline & & & & & & & $\begin{array}{c}\text { "AIR" } \\
\%\end{array}$ & $\begin{array}{c}\mathrm{CO}_{2} \\
\%\end{array}$ & $\begin{array}{c}\mathrm{CH}_{4} \\
\%\end{array}$ & $\begin{array}{c}\mathrm{C}_{2} \mathrm{H}_{6} \\
\%\end{array}$ & & & & \\
\hline & $13-1$ & 474 & 23.9 & 29,700 & -0.042 & 7.8 & & & & & & Hemipelagic mud & 2 & Early Pliocene \\
\hline & $17-1$ & 618 & 25.2 & 32,500 & -0.016 & 7.3 & 79.02 & 0.35 & 20.62 & 0.01 & & Hemipelagic mud & 2 & Late Miocene \\
\hline & $18-3$ & 637 & 24.9 & 29,700 & -0.043 & 7.8 & 41.90 & 0.47 & 57.60 & 0.03 & & Hemipelagic mud & 1 & Late Miocene \\
\hline & $19-1$ & 659 & 26.0 & 30,300 & -0.022 & 7.4 & & & & & & Hemipelagic mud & 2 & Late Miocene \\
\hline \multirow[t]{2}{*}{103} & $1-5$ & 6 & 25.5 & 34,100 & -0.043 & 7.9 & & & & & & Hemipelagic mud & 3 & Lower Pleistocene \\
\hline & $2-5$ & 45 & 25.3 & 31,400 & -0.040 & 7.8 & & & & & & Hemipelagic mud & 2 & Late Miocene \\
\hline$*$ & $3-3$ & 97 & NR & 31,400 & -0.050 & 8.0 & & & & & & Hemipelagic mud & 2 & Late Miocene \\
\hline$*$ & 4-NR & 170 & & & & & 36.00 & 1.10 & 63.00 & 0.01 & & Hemipelagic mud & 2 & Late Miocene \\
\hline$*$ & $5-3$ & 250 & NR & 29,700 & -0.061 & 7.9 & 41.00 & 0.60 & 58.00 & 0.02 & & Hemipelagic mud & 3 & Late Miocene \\
\hline \multirow[t]{8}{*}{104} & 2-NR & 36 & & & & & 44.35 & 2.16 & 53.44 & 0.01 & 0.04 & Hemipelagic mud & 2 & Late Miocene \\
\hline & $2-5$ & 42 & 23.2 & 31,500 & -0.033 & 7.5 & & & & & & Hemipelagic mud & 2 & Late Miocene \\
\hline & 3-NR & 62 & & & & & 31.11 & 1.89 & 66.96 & 0.01 & 0.02 & Hemipelagic mud & 2 & Middle Miocene \\
\hline & $3-4$ & 68 & 23.1 & 31,400 & -0.041 & 7.6 & & & & & & Hemipelagic mud & 2 & Middle Miocene \\
\hline & 4-NR & 133 & & & & & 37.69 & 2.55 & 59.75 & 0.01 & & Hemipelagic mud & 1 & Middle Miocene \\
\hline & $4-5$ & 139 & 23.0 & 30,800 & -0.059 & 8.0 & & & & & & Hemipelagic mud & 1 & Middle Miocene \\
\hline & 6-NR & 219 & & & & & 39.01 & 2.43 & 58.52 & 0.03 & & Hemipelagic mud & 1 & Middle Miocene \\
\hline & $6-5$ & 225 & NR & 27,500 & -0.057 & 7.9 & & & & & & Hemipelagic mud & 1 & Middle Miocene \\
\hline \multirow[t]{3}{*}{$*$} & 7-NR & 306 & & & & & 41.00 & 1.90 & 57.00 & 0.04 & & Hemipelagic mud & 2 & Middle Miocene \\
\hline & 8-NR & 401 & & & & & 40.91 & 2.88 & 56.18 & 0.03 & & Hemipelagic mud & 1 & Middle Miocene \\
\hline & $8-2$ & 402 & NR & 30,300 & -0.046 & 7.7 & & & & & & Hemipelagic mud & 1 & Middle Miocene \\
\hline$*$ & 9-NR & 495 & & & & & 41.00 & 3.20 & 56.00 & 0.04 & & Hemipelagic mud & 2 & Middle Miocene \\
\hline \multirow[t]{4}{*}{105} & $2-2$ & 32 & 26.1 & 34,100 & -0.027 & 7.3 & & & & & & Silty clay & 3 & Middle Pliocene \\
\hline & $3-4$ & 95 & 26.0 & 32,500 & +0.007 & 7.4 & & & & & & Clay & 3 & Early Pliocene \\
\hline & $8-4$ & 272 & 26.0 & 32,200 & +0.131 & 7.3 & & & & & & Silty clay & 3 & (barren) \\
\hline & $13-3$ & 326 & 25.5 & 32,400 & +0.016 & 7.6 & & & & & & Clay (up-hole corings) & 4 & $\begin{array}{l}\text { Middle } \\
\text { Cretaceous }\end{array}$ \\
\hline \multirow[t]{2}{*}{106} & $1-2$ & 1 & 23.0 & 34,100 & -0.005 & 7.5 & & & & & & Hemipelagic mud & 3 & Holocene \\
\hline & $3-4$ & 114 & 24.0 & 30,300 & +0.150 & 8.0 & & & & & & Hemipelagic mud & 3 & Late Pleistocene \\
\hline$* 106 \mathrm{~B}$ & $2-4$ & 455 & & & & & 42.00 & 0.30 & 58.00 & 0.01 & & Hemipelagic mud & 2 & Early Pliocene \\
\hline
\end{tabular}


TABLE $14-$ Continued

\begin{tabular}{|c|c|c|c|c|c|c|c|c|c|c|c|c|c|c|}
\hline \multirow[b]{2}{*}{ Hole } & \multirow[b]{2}{*}{ Core-Section } & \multicolumn{11}{|c|}{ Gas Chromatographyc } & \multirow[b]{2}{*}{ C.D. ${ }^{d}$} & \multirow[b]{2}{*}{ Age } \\
\hline & & $\begin{array}{l}\text { Deptha } \\
\text { (m) }\end{array}$ & $\begin{array}{c}\text { Temperature } \\
\left({ }^{\circ} \mathrm{C}\right)\end{array}$ & $\begin{array}{l}\text { Salinity } \\
\text { (ppm) }\end{array}$ & $\begin{array}{l}\text { Eh } \\
\text { (v) }\end{array}$ & $p \mathrm{H}$ & $\begin{array}{c}\text { "AIR" } \\
\%\end{array}$ & $\mathrm{CO}_{\%}$ & $\begin{array}{c}\mathrm{CH}_{4} \\
\%\end{array}$ & $\begin{array}{c}\mathrm{C}_{2} \mathrm{H}_{6} \\
\%\end{array}$ & $\begin{array}{c}\mathrm{H}_{2} \mathrm{~S} \\
\%\end{array}$ & Sediment Type & & \\
\hline * & $4-3$ & 756 & & & & & 44.00 & 0.50 & 50.00 & 0.04 & & Hemipelagic mud & 2 & Middle Miocene \\
\hline * & $5-2$ & 936 & & & & & 58.00 & 1.00 & 41.00 & 0.05 & & Hemipelagic mud & & Middle Miocene \\
\hline
\end{tabular}

*NOTE: Gas analysis taken from Table 2, Prelsey and Kaplan (this volume).

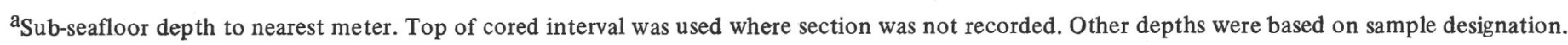

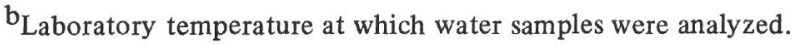

${ }^{c}$ Results are based on chromatograms of gas samples taken from gas pockets visible through the core liner. "Air" percentage is based on the chromatogram peak which records the combined effect of nitrogen, oxygen, and argon, and is interpreted as representing atmospheric contamination.

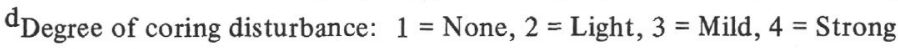

NR = Not Recorded. 


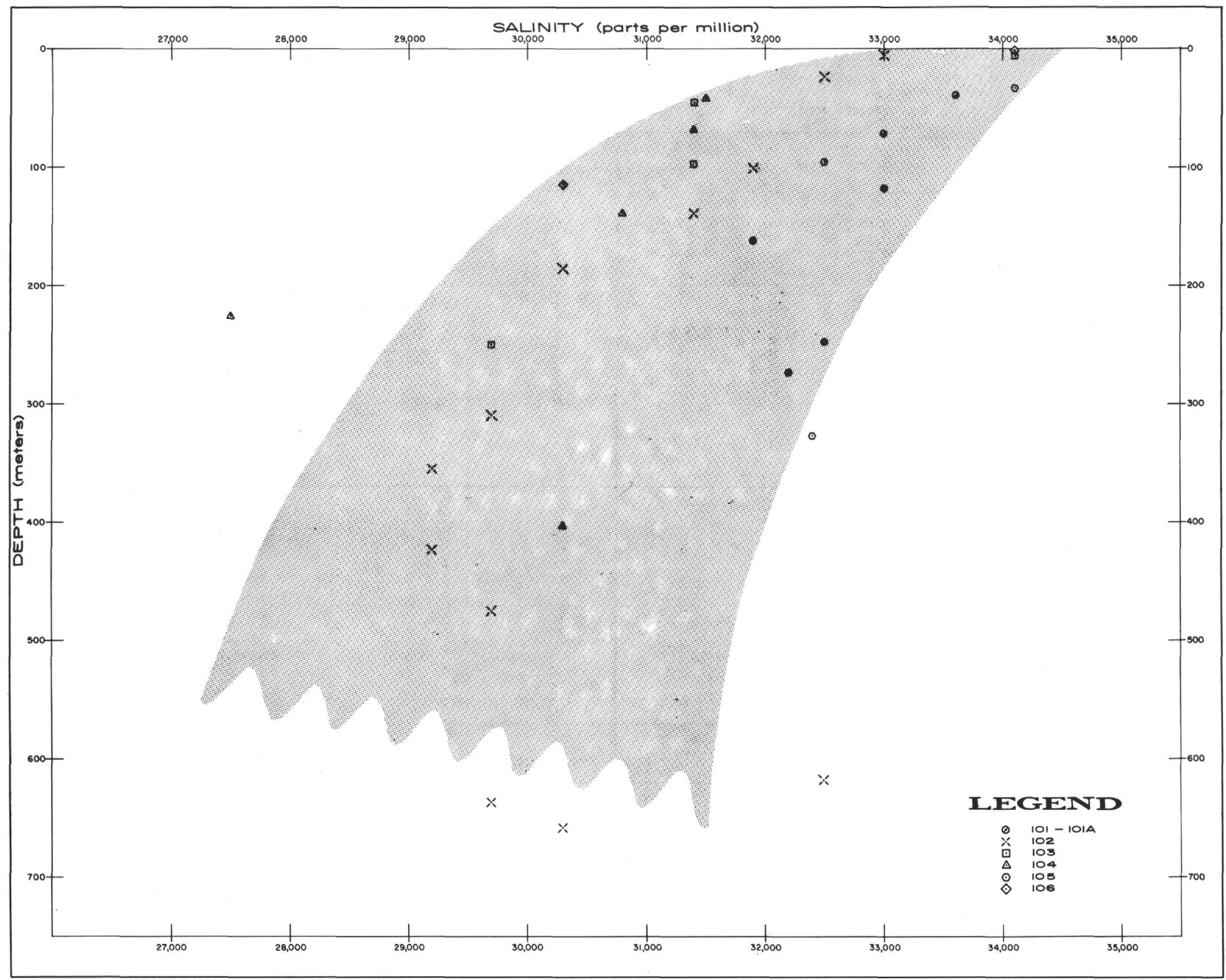

Figure 17. This cross plot of interstitial water salinity and sub-sea floor depth of sample shows a tendency for salinity to decrease as depth of sample increases. The shaded area identifies the general trend. The legend shows from which sites the cross plot data were obtained. The original data are listed in Table 15. 
degrees of compaction in mixtures of pure clay minerals and electrolyte solutions. It should be noted that Engelhardt and Gaida's experiments were unique in that they were the first to record salinity changes as they occurred during compression.

\section{GAS CHROMATOGRAPHY}

Gas chromatograms were recorded on board ship for 41 interstitial water samples and 19 gas samples. The 19 chromatograms of gas pocket samples from five cores at Site 102, two cores at Site 103, seven cores at Site 104, and three cores at Site 106 (Hole B) averaged about 44 per cent "air," 54 per cent methane, 1 per cent carbon dioxide, and a trace of ethane. The "air" percentage is based on the chromatogram peak which records the combined effect of nitrogen, oxygen, and argon, and is interpreted as representing atmospheric contamination. If the "air" value was removed from the analyses and the percentage recalculated, it would become readily apparent that the sediment gas is largely methane with an average value of about 97 per cent. Table 14 contains a complete listing of results of shipboard gas chromatograms which were run on gas pocket samples.

The Leg 11 gas pocket samples were collected on the coring deck by puncturing gas pockets visible through the core liner and allowing the gas to enter a sealed evacuated tube. The gas sample was then transferred to a basic gas chromatograph located in the shipboard chemist's laboratory. The percentage values for the gas constituents were obtained by summing the peak heights shown on the chromatograms using the arbitrary scale printed on the chromatograph paper and then determining what percentage of the sum is accounted for by each individual peak. As pointed out by Presley and Kaplan (this volume) this method:

... assumes that the chromatograph is equally sensitive to all gases, and should therefore be considered only a rough estimate of the actual composition of the injected gas. Furthermore, the injected gas may be quite different from the actual sample gas, because in this procedure there is no provision for either knowing or controlling the pressure of the gas. Thus, if the gas is under a reduced pressure, air will be sucked into the sampling syringe and become part of the injected sample.

A few samples have now been injected into a chromatograph for oxygen determination. If we make the reasonable assumption that this oxygen represents atmospheric contamination during sampling, the air peaks shown by the shipboard chromatograph are considerably too high. An adjustment would, however, little effect the $\mathrm{CH}_{4}$ to $\mathrm{C}_{2} \mathrm{H}_{6}$ to $\mathrm{CO}_{2}$ ratios, which are the primary concern in this study.
Methane gas was a prominent constituent of sediments at Sites 102, 103, and 104. Methane gas was also recovered from three cores at Site 106 (Hole B). Gas expansion within the core liner at Sites 102, 103, and 104 often caused the sediment to be extruded from the ends of the liner as it was removed from the core barrel. Continued gas expansion caused a segmentation of the core and the formation of several gas pockets between the segments. The pockets were recorded in the core description as voids (see Site Reports, this volume). The recorded positions of the voids and core segments do not necessarily represent the original locations of these elements since there was often considerable relocation during the cutting and opening of the core sections. There was a good correlation, however, between the occurrence of abundant voids and the occurrence of natural gas in the cores. The shallowest occurrence of voids due to gas expansion in cores from Sites 102, 103, and 104 was at 94 meters (Table 15). The deepest core recovery with no gas pockets was at 71 meters. The top of the gas-rich zone was therefore somewhere in the interval of 71 to 94 meters below the sea floor. The top of the zone appears to be a function of depth of burial rather than age of sediment (see Figure 3). Additional discussion of this relationship as well as the interrelationships between gas occurrence and the $p \mathrm{H}$ and $\mathrm{Eh}$ of the interstitial waters can be found elsewhere in this volume (Lancelot and Ewing).

\section{REFERENCES}

Abernethy, S. H., 1965. Improved equipment for a pulse method of sound velocity measurement in water, rock, and sediment. U. S. Navy Electronics Laboratory, San Diego, California, Technical Memorandum- 824 .

ASTM, 1965. Standard method of test for penetration of bituminous materials. American Society for Testing of Materials, Designation D-5-65. 1007.

Belding, H. F. and Holland, W. C., 1970. Bathymetric maps, eastern continental margin, U.S.A.: sheet 2 , Atlantic Ocean south of Cape Hatteras. Am. Assoc. Petrol. Geologists.

Boyce, R. E., 1968. Electrical resistivity of modern marine sediments from the Bering Sea. J. Geophys. Res. 75, 4759 .

Brier, C., Bennin, R. and Rona, P. A., 1969. Preliminary evaluation of a core scintillation counter for bulk density measurement in marine sediment cores. J. Sediment. Petrol. 39 (4), 1509.

Engelhardt. W. V. and Gaida, K. H., 1963. Concentration changes of pore solutions during compaction of clay sediments. J. Sediment. Petrol. 33, 919. 
TABLE 15

Sub-seafloor Depths of the Interval Containing Gas Pockets

in the Core Recovery From Sites 102, 103 and 104.

\begin{tabular}{ccccccccc}
\hline & \multicolumn{2}{c}{$\begin{array}{c}\text { Deepest Core With } \\
\text { No Gas Pockets }\end{array}$} & \multicolumn{2}{c}{$\begin{array}{c}\text { Shallowest Core } \\
\text { With Gas Pockets }\end{array}$} & \multicolumn{2}{c}{$\begin{array}{c}\text { Deepest Occurrence of } \\
\text { Gas Pockets }\end{array}$} \\
Site & Depth $(\mathrm{m})$ & Core & Age & Depth $(\mathrm{m})$ & Core & Age & Depth $(\mathrm{m})$ & Age \\
\hline 102 & 67 & 3 & $\begin{array}{l}\text { Early } \\
\text { Quaternary }\end{array}$ & 96 & 4 & $\begin{array}{l}\text { Early } \\
\text { Pleistocene }\end{array}$ & 661 (T.D.) & $\begin{array}{l}\text { Late } \\
\text { Miocene }\end{array}$ \\
103 & 48 & 2 & $\begin{array}{l}\text { Late } \\
\text { Miocene }\end{array}$ & 94 & 3 & $\begin{array}{l}\text { Late } \\
\text { Miocene }\end{array}$ & 449 (T.D.) & $\begin{array}{l}\text { Late } \\
\text { Miocene }\end{array}$ \\
104 & 71 & 3 & $\begin{array}{l}\text { Middle } \\
\text { Miocene }\end{array}$ & 133 & 4 & $\begin{array}{l}\text { Middle } \\
\text { Miocene }\end{array}$ & 617 (T.D.) & $\begin{array}{l}\text { Middle } \\
\text { Miocene }\end{array}$ \\
\hline
\end{tabular}

Evans, H. B., 1965. GRAPE-A device for continuous determination of material density and porosity. Trans. 6th Annual SPWIA Logging Sym. Dallas, Texas, II. B 1 .

Evans, H. B. and Cotterell, C. H., 1970. Gamma-ray attenuation density scanner. In Peterson, M. N. A. et al., 1970, Initial Reports of the Deep Sea Drilling Project. Volume II. Washington (U.S. Government Printing Office) Appendix II. 442.

Evans, H. B. and Lucia, J. A., 1970. Natural Gamma Radiation Scanner. In Peterson, M. N. A. et al., 1970, Initial Reports of the Deep Sea Drilling Project. Volume II. Washington (U.S. Government Printing Office), Appendix II. 440.

Hamilton, E. L., 1956. Low sound velocities in high porosity sediments. J. A coust. Soc. Am. 28, 16.

1959. Thickness and consolidation of deep sea sediments. Bull. Geol. Soc. Am. 70, 1399.

1960. Ocean basin ages and amounts of original sediments. J. Sediment. Petrol. 30, 370.

1963. Sediment sound velocity measurements made in situ from bathyscaph TRIESTE. J. Geophys. Res. 68, 5991.

1964. Consolidation characteristics and related properties of sediments from Experimental Mohole (Guadalupe Site). J. Geophys. Res. 69 (20), 4257.

1965. Sound speed and related physical properties of sediments from experimental Mohole (Guadalupe Site). Geophysics. 30, (2), 247.
1969. Sound velocity, elasticity, and related properties of marine sediments, north Pacific: II. Elasticity and elastic constants. Naval Undersea Research and Development Center, San Diego, Calif., T. P. 163.

1969b. Sound velocity, elasticity, and related properties of marine sediments, north Pacific: III. Prediction of in situ properties. Naval Undersea Research and Development Center, San Diego, Calif., T. P. 145.

1970. Sound velocity and related properties of marine sediments, North Pacific. $J$. Geophys. Res. 75, 4423 .

1971. Prediction of in situ acoustic and elastic properties of marine sediments. Geophysics, 32, 266.

Hamilton, E. L., Hucker, H. P., Keir, D. L. and Whitney, J. A., 1969. In situ determinations of the velocities of compressional and shear waves in marine sediments from a research submersible. Naval Undersea Research and Development Center, San Diego, Calif., T. P. 163.

Hamilton, E. L., Shumway, G., Menard, H. W. and Shipek, C. J., 1956. Acoustic and other physical properties of shallow-water sediments off San Diego. J. Acoust. Soc. Am. 28, 1.

Harms, J. C. and Choquette, P. W., 1965. Geologic evaluation of a gamma-ray porosity device. Trans. 6th Annual SPWLA Loggin Sym., Dallas, Texas VII, C].

Horn, D. R., Horn, B. M. and Delach, M. N., 1968. Sonic properties of deep-sea cores from the north 
Pacific basin and their bearing on the acoustic provinces of the north Pacific. Tech. Report No. 10, CU-10-68 NA VSHIPS N00024-67-C-1186.

Ingelman, K. R. and Hamilton, E. L., 1963. Bulk Densities of mineral grains from Mohole samples (Guadalupe Site). J. Sediment. Petrol. 33, 474.

Keller, G. H. and Bennett, R. H., 1970. Variations in the mass physical properties of selected submarine sediments. Marine Geol. 9, 215.

Kermabon, A., Gehin, C., Blavier, P. and Tonarelli, B., 1969. Acoustic and other physical properties of deep-sea sediments in the Tyrrhenian Abyssal Plain. Marine Geol. 7 (2), 129.

Koczy, F. F., 1963. Age determination in sediments by natural radioactivity In The Sea. M. N. Hill (Ed.). New York (Interscience). 3, 816.

Koczy, F. F. and Rosholt, J. N., 1963. Radioactivity in oceanography. In Nuclear Radiation in Geophysics. H. Israel and A. Krebs (Eds.). Berlin (SpringerVerlag) 18.

Lal, D. and Peters, B., 1967. Cosmic ray produced radioactivity on the earth. In "Cosmic Rays II": Encyclopedia of Physics. S. Flugge and K. Sitte (Eds.). New York (Springer-Verlag). 46, 551.

Laughton, A. S., 1954. Laboratory measurements of seismic velocities in ocean sediments. Proc. Roy. Soc. London, A. 222, 366.

; 1957. Sound propagation in compacted ocean sediments. Geophysics. 22, 233.

Lynch, E. J., 1962. Formation Evaluation. New York (Harper and Row). 422.

Mason, B. and Sand, L. B., 1960. Clinoptilolite from Patagonia. Am. Mineralogist. 45, 341.

Mauchline, J. and Templeton, W. L., 1964. Artificial and natural radioisotopes in the marine environment. In Oceanography and Marine Biology (An Annual Review). H. Barns (Ed.). London (George Allen \& Unwin Ltd.). 2, 229

Maxwell, A. E. et al., 1970. Initial Reports of the Deep Sea Drilling Project, Volume III. Washington (U.S. Government Printing Office).

Moore, D. G., 1964. Shear strength and related properties of sediments from experimental Mohole (Guadalupe Site).J. Geophys. Res. 69, 4271.

Moore, D. G. and Shumway, G., 1959. Sediment thickness and physical properties: Pigeon Point Shelf, California.J. Geophys. Res .64 (3), 367.

Nage, J. E. and Drake, C. L., 1957. Variation with depth in shallow and deep water marine sediments of porosity, density and the velocities of compressional and shear waves. Geophysics. 22, 523.
1963. Physical properties of marine sediments. In The Sea. M. N. Hill (Ed.). New York (Interscience). 3, 794

Paterson, N. R., 1956. Seismic wave propagation in porous granular media. Geophysics. 21, 691 .

Perry, D., 1970. Early diagenesis of sediments and their interstitial fluids from the continental slope, northern Gulf of Mexico. Trans. Gulf Coast Assoc. Geol. Soc., 20, 219.

Peterson, M. N. A. et al., 1970. Initial Reports of the Deep Sea Drilling Project, Volume II. Washington (U.S. Government Printing Office).

Poupon, A., Clavier, C., Dumanoir, J., Gaymard, R. and Misk, A. 1970. Log analysis of sand-shale sequences-a systematic approach. J. Petrol. Tech. $22,867$.

Prospero, J. M. and Koczy, F. F., 1966. Radionuclides in oceans and sediments. In Encyclopedia of Oceanography. R. R. Fairbridge (Ed.). New York (Reinhold Publishing Corp.). 730.

Rhodes, D. F. and Matt, W. E., 1966. Quantitative interpretation of gamma ray spectral logs Geophysics. 31, 410.

Richards, A. F., 1962. Investigations of Deep-Sea Sediment Cores, Part II, Mass Physical Properties, Technical Report 106. U.S. Navy Hydrographic Office. Washington D. C. 146.

Schlumberger, 1966. The formation Density Log. In Log Interpretation Principles, Schlumberger (Ed.). Paris (Kecran-Servant). 47.

Schreiber, B. C., 1968. Sound velocity in deep sea sediments. J. Geophys. Res. 73, 1259.

Shumway, G., 1958. Sound velocity vs. temperature in water-saturated sediments. Geophysics. 23, 494.

1960. Sound speed and absorption studies of marine sediments by a resonance method, Part I, Part II. Geophysics. 25, 451, 659.

Sutton, G. H., Berckheimer, H. and Nafe J. E., 1957. Physical analysis of deep sea sediments. Geophysics. 22,779 .

Terzaghi, K., 1925. Principles of soil mechanics II, Compressive strength of clays. Engineering News Record. 95, 796.

Terzaghi, R. D., 1940. Compaction of lime mud as a cause of secondary structure. J. Sediment. Petrol. 10,78 .

Weast, R. C., 1970-1971. Handbook of Chemistry and Physics. Cleveland (The Chemical Rubber Co.).

Weller, J. M., 1959. Compaction of Sediments. Bull. Am. Assoc. Petrol. Geologists. 43, 273.

Winokur, R. S. and Chanesman, S., 1966. A pulse method for sound speed measurements in cored ocean bottom sediments. U. S. Naval Oceanographic Office, I. M. 66-5, 10. 